\title{
DISSERTATION
}

\section{PHOTOGRAPH PRESENTATION ORDER AND RANGE EFFECTS IN VISUAL BASED OUTDOOR RECREATION RESEARCH}

\author{
Submitted by \\ Adam Wesley Gibson \\ Department of Human Dimensions of Natural Resources
}

\author{
In partial fulfillment of the requirements \\ For the Degree of Doctor of Philosophy \\ Colorado State University \\ Fort Collins, Colorado \\ Spring 2011
}

Doctoral Committee:

Advisor: Peter Newman

Paul Bell

Kurt Fristrup

Steve Lawson 


\title{
ABSTRACT \\ PHOTOGRAPH PRESENTATION ORDER AND RANGE EFFECTS IN VISUAL BASED OUTDOOR RECREATION RESEARCH
}

\begin{abstract}
Visual based research methods, referring to the use of visual images to represent recreation resource conditions, are commonly used in outdoor recreation research to investigate appropriate levels of visitor use. Visual methods were developed to allow for the simulation of recreation resource conditions that would be difficult to describe using narrative methods. The research contained in this dissertation builds on previous research related to visual based methods of outdoor recreation. While data from visual based research methods can provide a strong empirical basis to support outdoor recreation management decision-making, visual research methods applied in this context are subject to several potential sources of measurement bias. These potential sources of bias include effects associated with photograph presentation order, and effects associated with the range of resource conditions depicted. These two biases are respectively referred to as order effect and range effect.

This dissertation examines the issues of order and range effect biases in visual based outdoor recreation research. In a lab setting, respondents were
\end{abstract}


asked to rate a series of photographs of a recreation site in Rocky Mountain National Park in terms if the acceptability of the number of people at one time (PAOT) as depicted in the photographs.

In order to test for order effects, respondents were separated into six groups where photograph presentation order differed for each group. Results from qualitative and quantitative analyses indicate photograph presentation order significantly affected photograph acceptability ratings. In order to test for range effects, respondents were divided into seven groups where PAOT range differed for each group. Results from qualitative and quantitative analyses indicate range significantly affected photograph acceptability ratings.

Results from the investigations of order and range effects suggest a number of different principles that could be applied to future studies employing visual based methods. These principles are discussed along with future avenues of research that were uncovered through the course of the investigations of order and range effect biases. 


\section{ACKNOWLEDGMENTS}

I would like to thank my advisor and committee members for your direction and constant support. I would also like to thank United Airlines for not allowing the use of cell phones in flight. Otherwise I might not have gotten a good opportunity to explore dissertation topics with my excellent yet overworked advisor. I would like to thank Kurt for being an extremely difficult and stubborn scientist. You made me want to quit, but you also made me write a damn good dissertation (or better at least). I would like to thank my friends and family for your unwavering support and for constantly asking me, "When are you going to finish?" I can't wait until one of you tries to get a PhD. Lastly, l'd like to thank my girlfriend Lauren. You more than anyone know what a trial this has been, and I thank you for your support all along the way. I know you always wanted to marry a doctor, well maybe now you'll get your chance. 


\section{DEDICATION}

I would like to dedicate this dissertation to my parents. I suppose my journey through academia was much like the tortoise and less like the hare. I didn't do particularly well at any point in the process, but I just kept on truckin'. As much as I would like to take credit for this achievement, I know that my perseverance and predilection towards learning was something you infected me with early on in my life. Thank you. I love you guys and I hope I can make you proud. I miss you Pop. 
ABSTRACT OF DISSERTATION................................................... ii

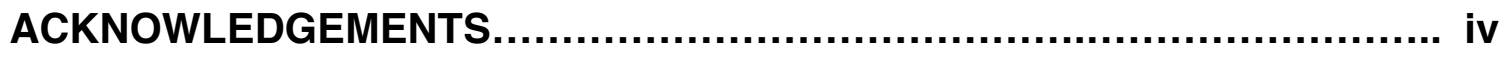

DEDICATION.......................................................................... v

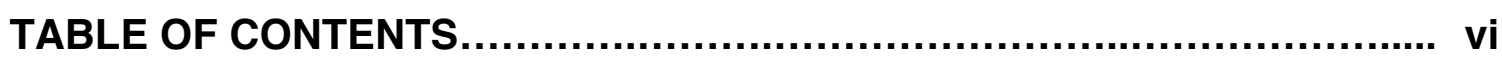

CHAPTER I: Visual Based Outdoor Recreation Research................... 1

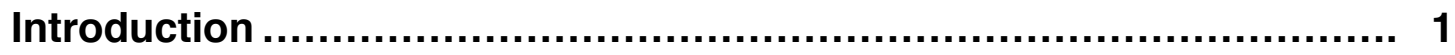

Overview of Dissertation................................................. 3

Review of Literature................................................................ 4

Theoretical Basis of Visual Methods................................... 4

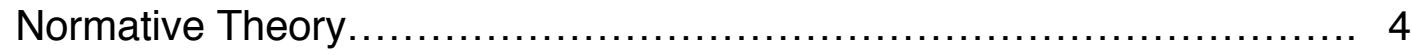

Normative Outdoor Recreation Research............................. 5

Measuring Evaluative Standards....................................... 6

Evaluative Standards and Visual Based Methods......................... 9

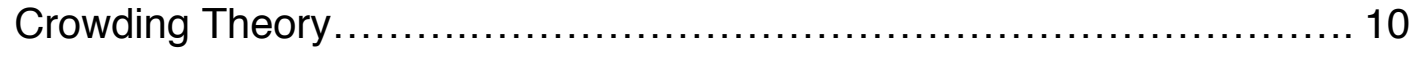

Combining Normative and Crowding Theory Into Visual Based ........... 11

Methods

History of Visual Based Methods........................................ 12

Visual Based Methods in Outdoor Recreation Research................... 12

Potential Bias Within Visual Based Methods.............................. 12

Order Effect Bias in Visual Based Outdoor Recreation ................... 16 Research

Range Effect Bias in Outdoor Recreation Research...................... 18

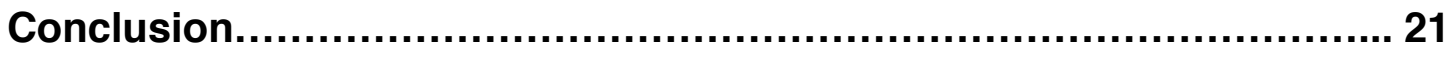

CHAPTER II: An Investigation of Photograph Presentation Order .......... 22 Effects in Visual Based Methods of Outdoor Recreation

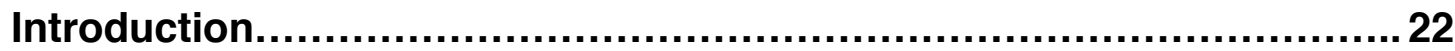




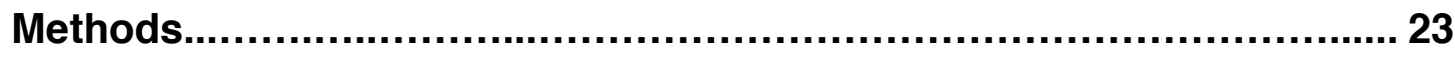

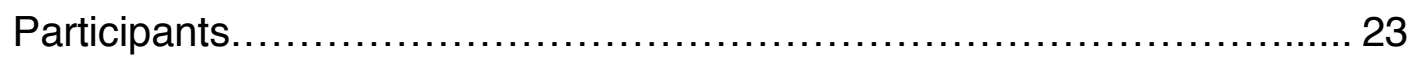

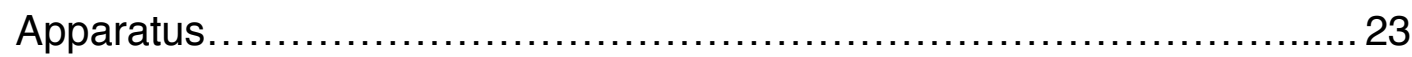

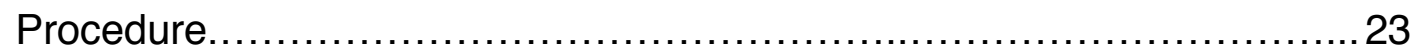

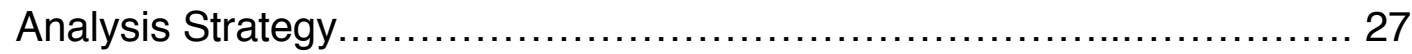

Non-Monotonic Value Summary......................................... 27

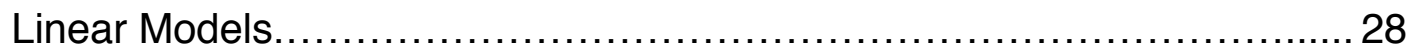

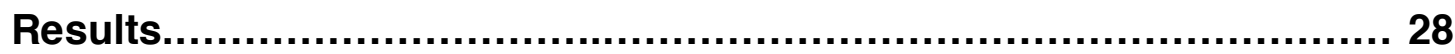

Non-Monotonic Values..................................................... 30

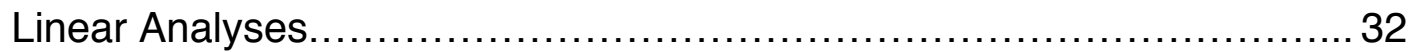

Model 1 - Photograph Acceptability and Photograph Number............... 34

Model 2 - Photograph Acceptability and Photograph Number .............. 34 with Presentation Order and Individual Idiosyncrasy Model 3 - Photograph Acceptability and Photograph Number ............. 34 with Presentation Order and Individual Idiosyncrasy

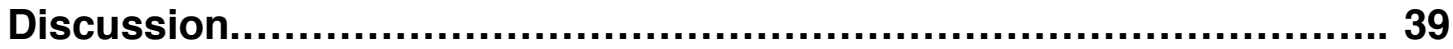

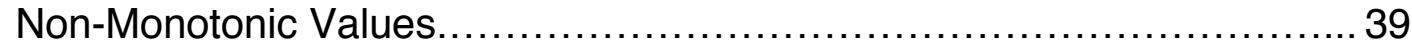

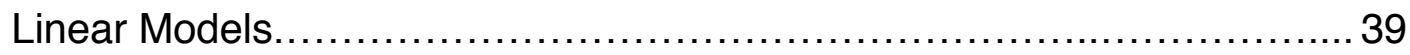

Limitations............................................................ 40

Conclusions.............................................................................. 41

CHAPTER III: An Investigation of Photograph Range Effects in ............. 43 Visual Based Outdoor Recreation Research

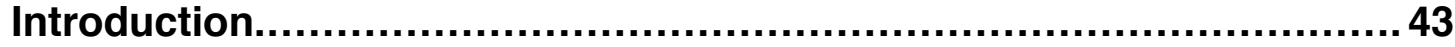

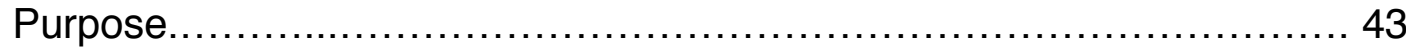

Methods.............................................................................. 44

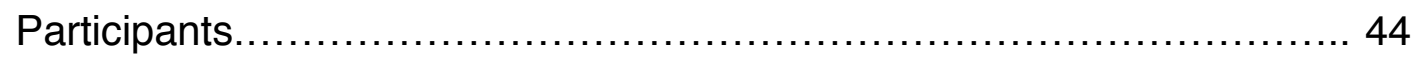

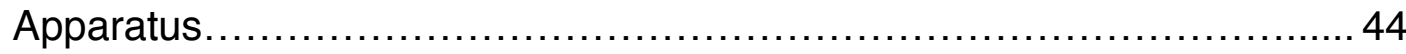

Procedure..................................................................... 45

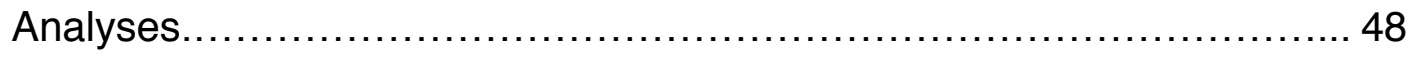

Results.......................................................................... 48

Linear Models.................................................................. 51

Model 1 - Photograph Acceptability and $\log _{10}$ PAOT...................... 51 
Model 2 - Photograph Acceptability and $\log _{10}$ PAOT and Range Group

Model 3 - Photograph Acceptability and $\log _{10}$ PAOT and 52 Photograph Sequence Number

Discussion........................................................................... 55

Linear Models.................................................................... 55

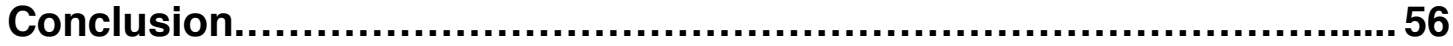

CHAPTER IV: Future Visual Based Outdoor Recreation Research............59

Summary of Findings.................................................................. 59

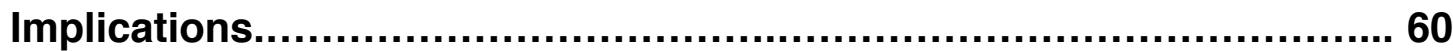

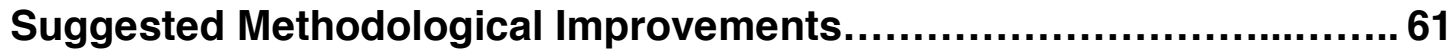

Limitations and Future Research................................................6 64

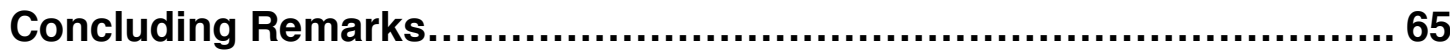

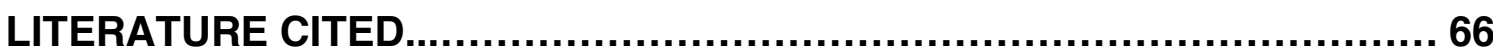

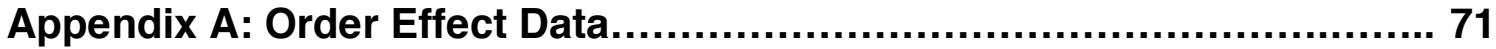

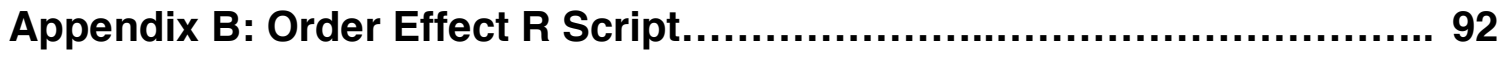

Appendix C: Range Effect Data....................................................... 98

Appendix D: Range Effect R Script............................................ 129 


\section{CHAPTER I}

\section{Visual Based Outdoor Recreation Research}

\section{Introduction}

Outdoor recreation research is an area of social science that incorporates the study of human behavior into the broader effort of natural resource conservation. The science of outdoor recreation research "recognizes that conservation is about people as much as it is about species or ecosystems" (Mascia, Brosius, Dobson, Forbes, Horowitz, McKean, \& Turner, 2003, p. 649).

One of the fundamental reasons for incorporating social science into natural resource conservation efforts is the ability of social science to identify behavioral standards that land managers can use to establish acceptable conditions (Heywood, 1996; Shelby \& Vaske, 1991). For example, information regarding public attitudes toward resource and visitor experience conditions can help land managers identify thresholds of acceptability, which can then inform management policies.

Visual based methods of outdoor recreation research are one of the most common techniques utilized for providing land managers with information regarding acceptable resource and visitor experience conditions (Manning, 2007). Visual based methods refer to the use of photographs or other visual representations to simulate existing environments in an evaluative questionnaire (Manning, 2007). Within the visual method, images can be evaluated on social, biological, or managerial characteristics. The most common use of visual based 
methods is in the evaluation of visitor use density, also called PAOT and PPV, people at one time and people per viewscape respectively (e.g., Manning, Lime, Hof, \& Freimund, 1995; Manning, Lime, Freimund, \& Pitt, 1996; Manning, 1999; Jacobi \& Manning, 1999; Manning, Valliere, Minteer, Wang, \& Jacobi, 2000; Manning, Lawson, Newman, Laven, \& Valliere, 2002; Manning, Wang, Valliere, Lawson, \& Newman, 2002; Manning, Valliere, Wang, Lawson, \& Newman, 2003; Valliere \& Manning, 2003; Manning, Freimund, \& Marion, 2004; Manning, Leung, \& Budrul, 2005; Manning, 2007). When used to evaluate PAOT, visual methods provide information that can be helpful in identifying thresholds of visitor use beyond which visitors feel unacceptably crowded, which in turn, provides one bases of information for forming use limits within protected areas.

Visual based methods have been used for over 25 years (Shelby \& Harris, 1985) with few refinements. However, the lack of refinements in visual based methods is not for lack of trying. Manning and Freimund (2004) provided the most comprehensive review of visual based methods and found that "careful applications [of visual based methods] do not appear to be heavily influenced by methodological variations" (p. 572). Furthermore, "findings from studies employing visual research methods generally meet conventional tests of research validity" (Manning \& Freimund, 2004, p. 573). Visual based methods were investigated my Manning and Freimund (2004) in order to strengthen results from the last 30 years of visual based outdoor recreation research. 
However, despite the efforts of Manning and Freimund (2004), the validity of visual based methods of outdoor recreation research is still in question. As will be discussed in detail, potential biases of visual based methods still exist. Consequently, the data from conventional applications of visual based studies may not be suitable for use in support of decision-making efforts.

\section{Overview of Dissertation}

The purpose of this dissertation was to build on previous research from Manning and others, and further investigate potential biases within visual based methods of outdoor recreation research. More specifically, this dissertation focused on two potential biases hereafter referred to as order effect and range effect. Order effect bias refers to the effect random photograph presentation orders may have on photograph acceptability ratings, and range effect bias refers to the effect PAOT range depicted in photographs may have on photograph acceptability ratings. Both of these biases will be discussed in greater detail later in the chapter.

The remainder of Chapter I focuses on a review of literature relevant to the investigation of visual based methods of outdoor recreation research. Chapter II investigates the existence of order effect bias in visual based methods. Chapter III investigates the existence of range effect bias in visual based methods. Finally, Chapter IV provides the broader implications of the research contained in this dissertation by suggesting methodological imporvements that can be employed by researchers when using visual based methods in future research. 
Chapter IV also discusses future avenues of research suggested by the investigations of order and range effect.

\section{Review of Literature}

Theoretical Basis of Visual Methods

Visual based methods, as they are currently used in outdoor recreation research, are the product of decades of research. Visual based methods combine digital photography technology with social science theory into one survey tool. The result is an inexpensive, adaptable, and simple method for obtaining large amounts of evaluative data from survey respondents. In order to truly understand how visual based methods work, we must understand where they came from.

\section{Normative Theory}

Some of the earliest implementations of visual methods were in the field of sociology. Visual based methods are the operationalization of sociological theory known as normative theory. As the name suggests, "norms" are used to describe social regularities and socially appropriate behavior (Parsons, 1951; McDonald, 1996). In this sense, norms existed only at the social level and could not be reduced to the individual. Later norms were used to describe not only how people act, but also how they should act (Homans, 1950). The introduction of injunctive norms led to the conceptualization of norms as obligatory (Gibbs, 1977), and the obligatory nature of norms prompted social scientists to begin examining the role of the individual in the social process of norms. 
From a psychological perspective, norms also involve the cognitive processes of the individual. For example, Fishbein and Ajzen (1975) argued that not only is it important what society thinks is appropriate behavior, but what the individual thinks society thinks is appropriate behavior. Similarly, McDonald (1996) states that from the psychological perspective, "individuals comply with norms because of what they anticipate receiving or what they may lose if they violate the norm" (p. 2). The components of norms as outlined by sociology and social psychology collectively are that norms (1) are obligatory and enforced by sanctions; (2) guide behavior; and (3) are shared by social groups (McDonald, 1996).

\section{Normative Outdoor Recreation Research}

Normative theory, as outlined above, has been adapted for use in outdoor recreation research. In recreation research, norms have been conceptualized as shared attitudes and preferences towards a particular recreation setting characteristic (social, environmental, or managerial), also called a "normative variable" (Manning, 2007). The major differences between the outdoor recreation and sociological conceptualization of norms are that outdoor recreation research norms (1) include emerging norms, which may not involve fully developed sanctions; (2) can apply to conditions (social and environmental) as well as behavior; and (3) often regulate collective rather than individual behavior (Manning, Lawson, \& Frymier, 1999). The differences between the sociological and recreational norm concept form the basis of a debate regarding the true 
nature of norms, and whether or not what outdoor recreation researchers call norms are actually norms.

Regardless of whether or not evaluative standards measured by recreation researchers are actually norms, research of recreation evaluative standards can still be of utility to the management of parks and protected areas. Shelby, Vaske, and Donnelly (1996) contend that normative research is useful to recreation managers because evaluative standards can (1) help managers identify desirable conditions; (2) define salient recreation variables or indicators;

(3) inform standards for indicator variables; (4) differentiate between unacceptable and acceptable conditions; and (5) indicate the degree of consensus about normative variables.

\section{Measuring Evaluative Standards}

Methods for measuring evaluative standards have been derived based on the work of Jay Jackson's return potential model (Heberlein, 1977; Manning, 1985; Shelby \& Heberlein, 1986; Shelby, Vaske, \& Donnelly, 1996; Vaske, Graefe, Shelby, \& Heberlein, 1992; Vaske, Donnelly, \& Shelby, 1993). Jackson's model allows for the analysis of specific characteristics of norms through the use of return potential curves (Jackson, 1965), also referred to as acceptability curves. More specifically, acceptability curves are graphical representations of measures of social acceptability created through the aggregation of personal acceptability of resource conditions. 
For example, Figure 1-1 displays a curve associated with the acceptability of various PAOT levels for a particular site. As seen in Figure 1-1, PAOT is located on the $\mathrm{x}$-axis (this can be any resource, social, or managerial setting variable, such as the size of fire rings, trail width, behavior of groups, etc.), while the acceptability corresponding with PAOT is located on the $y$-axis. The numbers on the $x$-axis can vary because they correspond to the range of PAOTs in the photographs used, but conventionally the range of acceptability is a 9-point scale where $-4=$ very unacceptable, $4=$ very acceptable, and $0=$ neither . 


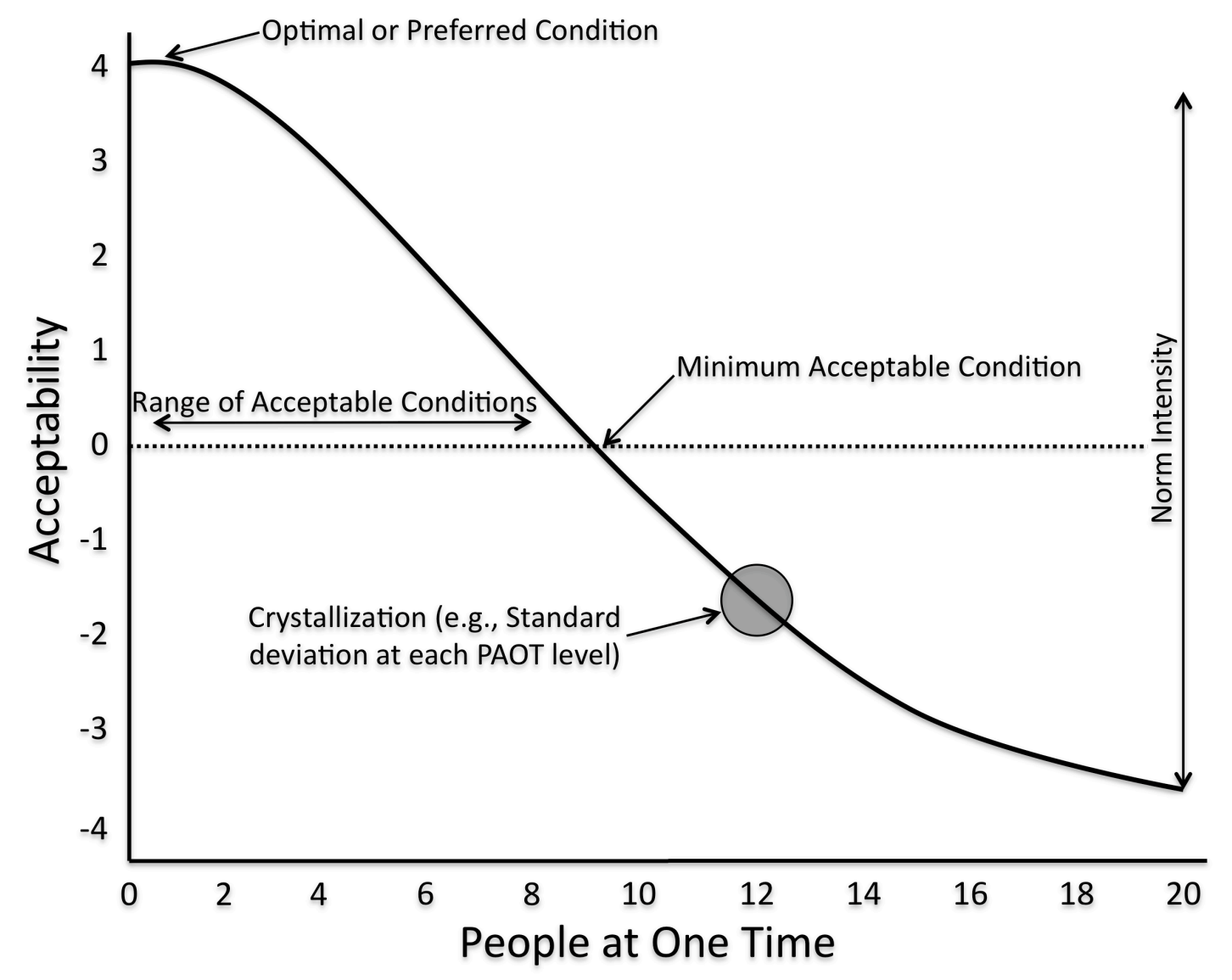

Figure 1-1. Example of a hypothetical curve showing the acceptability of various levels of PAOT (Source: Manning, 1999).

Once an acceptability curve is created, characteristics of the curve can provide helpful information to managers (Manning, 2007). For example, acceptability of PAOT can help inform managers about appropriate use-levels for a particular area. The height of the curve (norm intensity) can inform managers how important PAOT is to visitors, where the broader the range in acceptability ratings, the more salient PAOT is to visitors. The highest point on the curve relates to the optimal or preferred PAOT condition. The PAOT range corresponding to acceptability ratings greater than 0 reflect the range of acceptable conditions. The point where the curve crosses the neutral line 
describes the minimum acceptable condition. And finally, the standard deviation at each point on the curve is a measurement of crystallization, which can inform managers about the level of respondent agreement about the acceptability of each individual level of PAOT.

\section{Evaluative Standards and Visual Based Methods}

Visual based methods are a way of measuring evaluative standards through the use of acceptability curves. For example, in the use of visual methods to measure acceptability of PAOT, photographs are presented to respondents that represent a range of possible resource conditions (e.g., various levels of PAOT). Respondents evaluate the photographs in terms of acceptability of PAOT. Results are then aggregated into acceptability curves representing the social acceptability of the various PAOT levels.

When investigating acceptability of PAOT, measurement of evaluative standards provide the answers as to how visual based methods work, but not exactly why visual based methods work. In other words, measurement of evaluative standards provide the methodology for measuring acceptability of PAOT, but not the theoretical basis explaining the relationship between acceptability and PAOT. For the answers as to why visual based methods work in the measurement of acceptability of PAOT, we have to turn to a sociological theory known as crowding theory. 


\section{Crowding Theory}

Crowding, defined as the negative evaluation of density (Manning, 1999), involves two components. The first component is descriptive, and the second component is evaluative (Shelby, Vaske, \& Donnelly, 1996). For example, physical characteristics of the setting (e.g., environmental, managerial and social characteristics) and objective observations about the encounters (e.g., PAOT) would be considered aspects of the descriptive component. The psychological reactions (e.g., whether the person perceives the conditions as crowded) to the descriptive components would be considered aspects of the evaluative component (Manning, Lime, Freidmund, \& Pitt, 1996; McDonald, 1996; Heywood, 1996; Manning, 1999; Manning \& Lawson, 2002).

Shelby (1980) developed a model (further refined by Manning, 1999) describing the relationship between the descriptive and evaluative components of crowding, and linked them to the larger idea of recreation satisfaction, defined as the congruence between expectations and outcomes. The main idea of the crowding model is that use levels of a recreation area are related to recreation satisfaction through evaluations of the actual density of people (Figure 1-2). In other words, PAOT can lead to the perception of crowding, which can then lead to decreased satisfaction with the recreation experience. 


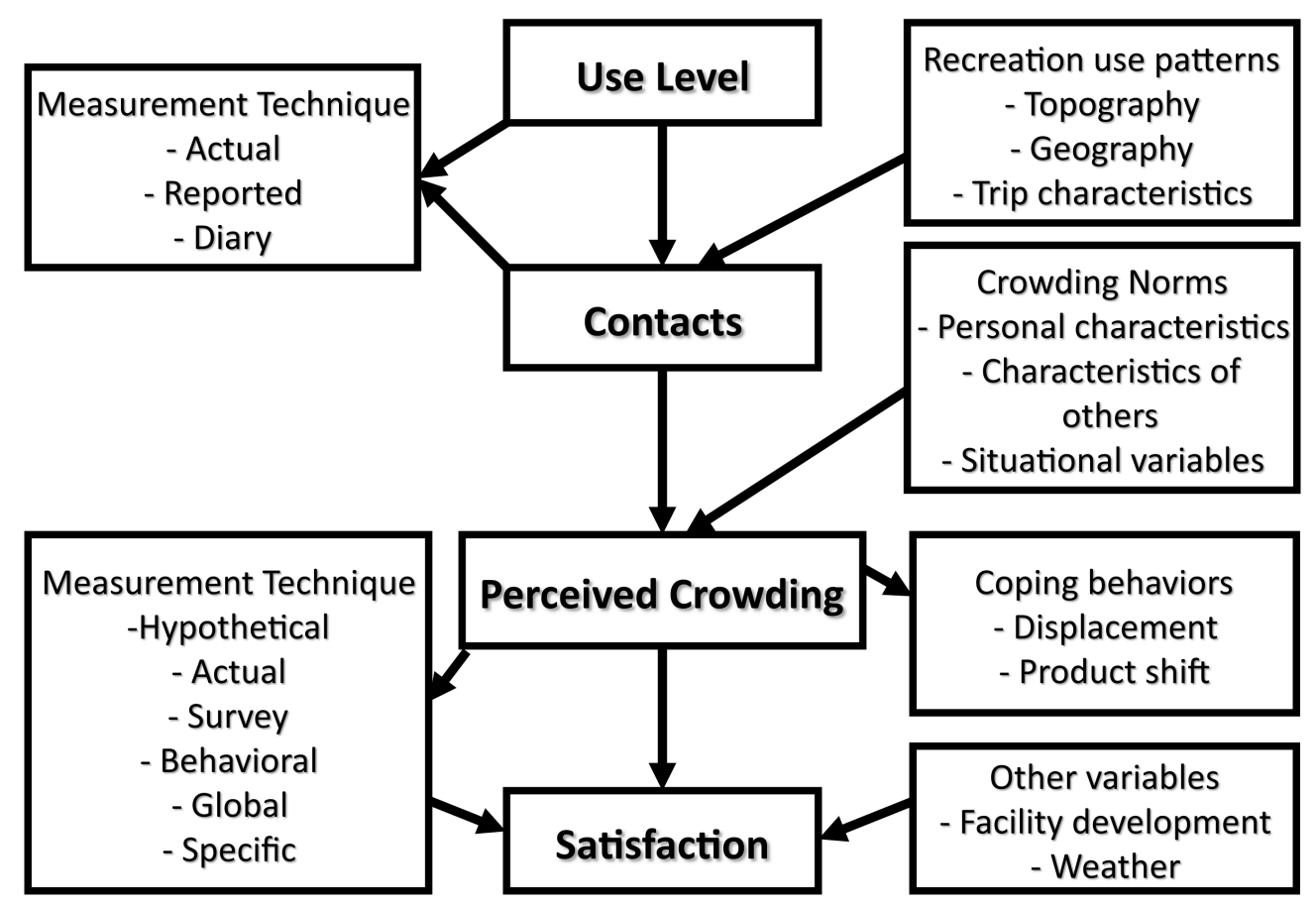

Figure 1-2. Crowding model (Source: Manning, 1999, p. 94).

Combining Normative and Crowding Theory Into Visual Based Methods

Visual methods can be used to measure the acceptability of PAOT due to the normative nature of PAOT, and the theoretical relationship between crowding and recreation satisfaction. Crowding theory provides support for why the relationship between PAOT and acceptability exists. Normative theory provides the means by which acceptability of PAOT can be measured and aggregated across individuals (i.e., acceptability curves). In other words, normative theory and crowding theory combine to provide the justification for using visual based methods to measure acceptability of PAOT. However, investigating acceptability of PAOT was not the purpose for which visual based methods were originally created. 


\section{History of Visual Based Methods}

The use of visual images originates from the field of environmental psychology, where simulations are routinely used in order to investigate human responses to yet to be built environments (Bateson \& Hui, 1992; Bosselmann \& Craik, 1987; Hershberger \& Cass, 1974; McKechnie, 1977). Visual based methods were first utilized in a natural resource related study to investigate the acceptability of ecological impacts to landscapes and campsites (Chenoweth, 1990; Shelby \& Harris, 1985; Shelby \& Shindler, 1992). Visual methods were adapted by recreation researchers in order to measure the acceptability of a range of recreation resource conditions that would be difficult to replicate in real life (Manning \& Freimund, 2004).

\section{Visual Based Outdoor Recreation Research}

In recreation research, visual methods are often used in place of narrative methods, because visual methods are able to easily capture variables that would be awkward to describe (Manning, 2007; Manning \& Freimund, 2004). The low cost and ease of creating digitally manipulated photographs make visual methods an attractive methodology for protected area managers and researchers alike (Freimund, Vaske, Donnelly, \& Miller, 2002). Visual methods also allow for easily repeatable treatment conditions (Manning, Lime, Freimund, \& Pitt, 1996).

Potential Bias Within Visual Based Methods

Visual based methods have been used extensively, and previous research supports the validity of visual methods (Manning \& Freimund, 2004). Context 
bias, placement bias, and starting point bias have all been examined in research investigating the validity of visual based methods (Manning \& Freimund, 2004; Manning, Lawson, Newman, Laven, \& Valliere, 2002; Manning, Valliere, Wang, \& Jacobi, 1999). In all these cases, the conclusion has been that the visual method is free from significant bias. However, previous research may not have investigated deeply enough to uncover well-hidden biases inherent in the visual based methodology.

One reason to think that results from visual based studies are somehow biased concerns the similarities between acceptability curves from visual based studies. For example, Figure 1-3 displays acceptability curves from four separate studies investigating acceptability of PAOT (Manning, 2007, p. 160; Manning, 2007, p. 188; Manning \& Freimund, 2004, p. 570; Manning, Lawson, Newman, Laven, \& Valliere, 2002). In each study, depicted PAOT increased in each photo (x-axis) and respondents were asked to rate the acceptability of each photo on a 9-point scale (y-axis) ranging from -4 (Very Unacceptable) to +4 (Very Acceptable). 


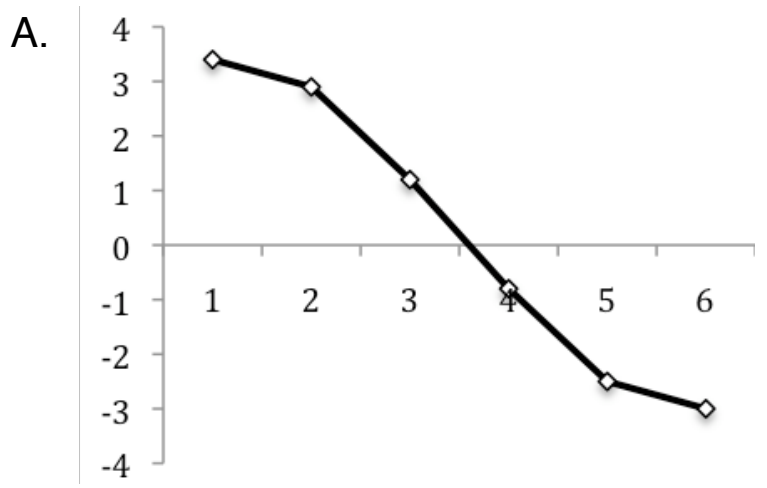

Muir Woods National Monument

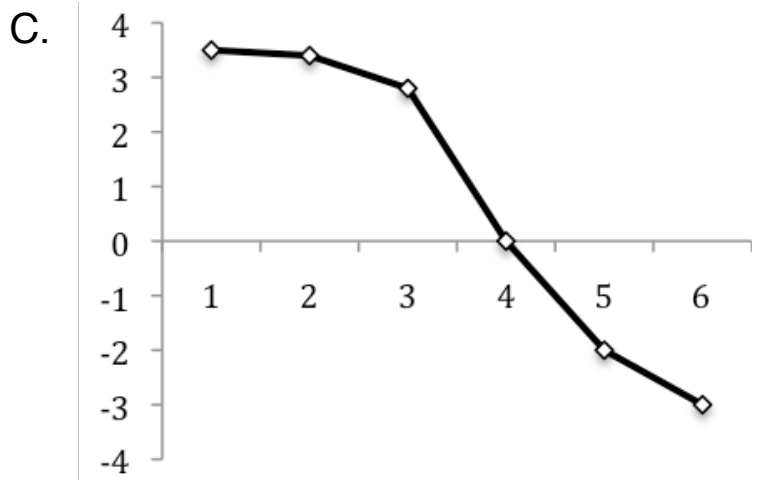

Alcatraz Island National Monument

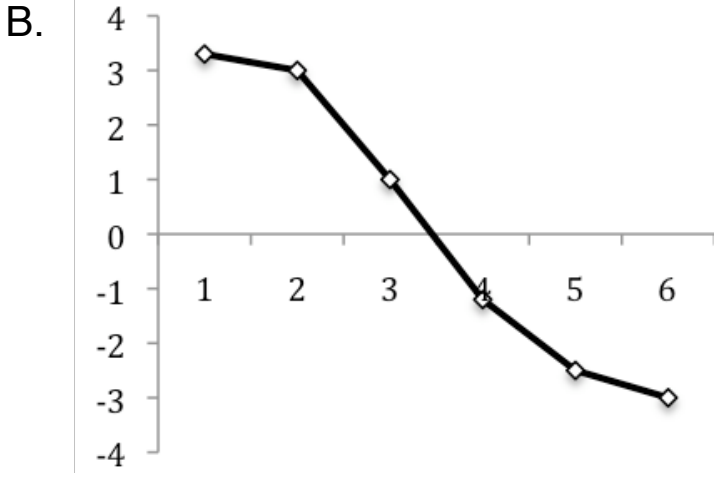

Mesa Verde National Park

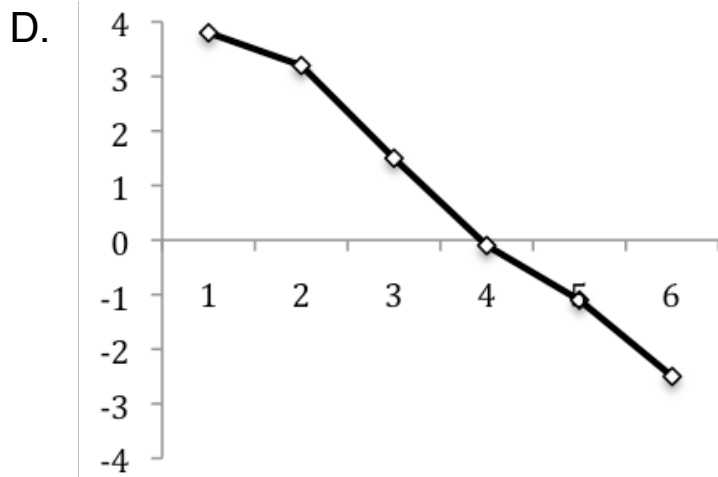

Grand Canyon National Park

Figure 1-3. Examples of acceptability curves from (A) Manning, 2007, p. 160; (B) Manning, 2007, p. 188; (C) Manning \& Freimund, 2004, p. 570; And (D) Manning, 2007, p. 160.

As seen in Figure 1-3, the norm curves are qualitatively similar in shape and intensity even though the studies were conducted in a wide variety of parks (Muir Woods National Monument, Mesa Verde National Park, Alcatraz Island National Monument, and Grand Canyon National Park) depicting a large range of PAOT (the smallest PAOT range was 18 , while the largest range was 110). In each acceptability curve, there is a downward trend in acceptability from one photograph to the next, and the curve crosses between photographs 3 and 4 . 
Acceptability curves from visual based studies could look similar for any number of reasons. Simply because they appear similar is no definite indication of bias. After all, the quantitative results derived from each of the curves depicted in Figure 1-3 are different. For example, PAOT varied from 0 to 110 in Curve B, and from 0 to 16 in Curve D. Therefore, the minimum acceptable condition suggested is about 60 PAOT for Curve $B$, and about 8 for Curve $D$.

The visual similarity in appearance of acceptable curves could be a product of using the same methodology. The range of conditions depicted in visual method photographs generally goes from "good" to "bad", so it may not be surprising that the curves always cross in the middle of the presentation sequence. However, the rigorous adherence to consistent methods may be a detriment to visual based methods rather than a benefit.

Visual based methods typically utilize a five or six-photograph set depicting resource conditions. All respondents see every photograph. All respondents also see the photographs in the same presentation order, either all at once, or as is more often the case, one at a time in increasing amounts of the normative variable (e.g., increasing PAOT). These two elements of the visual method, presenting all photographs and presenting photographs in the same order, may be two aspects of the conventional application of visual methods that bias acceptability ratings. For example, would results from the study depicted in Curve B in Figure 1-3 have been the same if the photographs had been 
presented in random orders to respondents? Furthermore, would a PAOT of 60 be rated the same in a sequence of photos ranging from 30 to 90 or 60 to $120 ?$ The questions raised respectively refer to order effect bias and range effect bias. Order effect bias and range effect bias have not been investigated in previous research of visual based methods.

\section{Order Effect Bias in Visual Based Outdoor Recreation Research}

Economists using contingent valuation research methods have investigated biases similar to those of order effect bias. Contingent valuation techniques were originally developed to estimate the value of nonmarket services or products such as air, water, wildlife, or recreation using the idea of "willingness to pay" (Thayer, 1981). In this context, "the value [an] individual places on the nonmarket commodity may be influenced by the point at which the bidding commences" (Thayer, 1981, p. 28). This bias is commonly known as starting point bias.

Starting point bias may function in a similar way in the context of photograph evaluations used in the visual method. However, it may not only be important when the "bidding" commences (which photograph is presented first in this case), but how the "bidding" proceeds (the entire photo presentation sequence). For example, respondents may evaluate a photo with high PAOT very differently if they were previously exposed to photos with high PAOT than if they were exposed to photos with low PAOT. Order effect bias is an expanded 
version of starting point bias that incorporates the full progression of photograph presentation order, not simply where the presentation order began.

Question-order effects have been researched in the field of political science. Bradburn and Mason (1964), McFarland (1981), and Moore (2002) all investigated the effect of question order on response variance in political questionnaires. Political questionnaires are not equivalent to normative recreation questionnaires given the potential for rapid change in political opinions with the emergence of a relevant news story, but political science studies of order effects can still be informative. Results from Moore (2002) revealed that question order can have a number of different effects on respondent evaluations to survey questions (e.g., additive, subtractive), and that questions vary in their susceptibility to order effects (McFarland, 1981).

Order effect bias of visual based recreation research methods has received little attention in previous studies. Manning et al. (2002) tested one aspect of order effect bias of photo evaluations by reversing the order in which photos are typically presented to respondents. The authors reversed the photo presentation order (presenting photos with high PAOT first instead of last) to test whether starting at the other extreme of the photo evaluation sequence had any effect on the acceptability ratings of photos. The results of the study found significant differences in acceptability ratings based on presentation order (2 out of 6 pair-wise comparisons were significantly different), but those differences were substantively small. Thus, Manning et al. concluded that the number of 
people depicted in the photos was predicting photo acceptability ratings, and not photo presentation order.

However, Manning et al. (2002) was not a complete investigation of ordereffect bias and fell short of answering the question of whether or not photo presentation order affects photo acceptability ratings when using visual based methods. Random photograph presentation sequences are required to fully test whether or not photograph presentation order affects evaluations of PAOT depicted in photographs. The comparison of evaluations from photograph presentation sequences that start with every possible level of PAOT and progress randomly through the levels of PAOT would provide a thorough test for the existence of order effect bias and is the design approach used in this dissertation. But, presentation order is not the only variable that may be biasing acceptability ratings of photographs. The range of depicted conditions may also be biasing acceptability ratings.

Range Effect Bias in Outdoor Recreation Research

Like order effect bias, range effect bias has received little attention in past outdoor recreation research. In fact, range effect, referring to the varying effect the chosen range of depicted conditions may have on acceptability ratings, has not been investigated in any previous visual method outdoor recreation research, though closely related research may be informative. Schwarz, Hippler, Deutsch, and Strack (1985) is a seminal paper concerning response scales. Schwarz et al. (1985) contend that respondents "use the frame of reference provided by the 
scale in estimating their own behavior" (p. 394). The authors make this conclusion because they found that respondents stating they watched more than 2.5 hours of television could vary up to $37 \%$ by altering survey response categories.

Range effect does not refer to the response scale (the response scale is generally -4 to +4 ), but response scale effects could translate the range of photographs presented. Hall and Roggenbuck (2002) explain:

"A question asking about the acceptable number of encounters per day might be anchored with 0 to 20 or might be anchored with 0 to 100 . If respondents truly have a preexisting notion about encounters, and this is an important issue to them, the distributions of responses should change little across the two formats. If on the other hand, the opinion about this issue is constructed on the spot (Zaller \& Feldman, 1992), the mean should be substantially higher on the second version, because respondents are reacting to the categories offered, rather than on the basis of a genuine personal preference or 'norm'." In the preceding excerpt, Hall and Roggenbuck (2002) have summarized the essence of range effect bias.

According to Hall and Roggenbuck (2002), the effects of range bias are manifested in the shapes of acceptability curves. That is to say, if the independent variable range did affect acceptability ratings, the acceptability curve 
will have a shape different than typical acceptability curves (Figures 1-1 and 1-3). For example, if respondents evaluated a series of photos depicting a range of PAOT from 0 to 42, the range of PAOT might have had little effect if acceptability curves from any subset range (e.g., 0-10, 0-20, 15-42) overlapped with the acceptability curve of the whole PAOT range. A lack of differences in acceptability curves would suggest that that acceptability is not affected by differences in PAOT range. However, if the acceptability curves from subset ranges did not overlap and were distinct segments, these differences might be attributed to the range of PAOT in the presented photos (Figure 1-4).

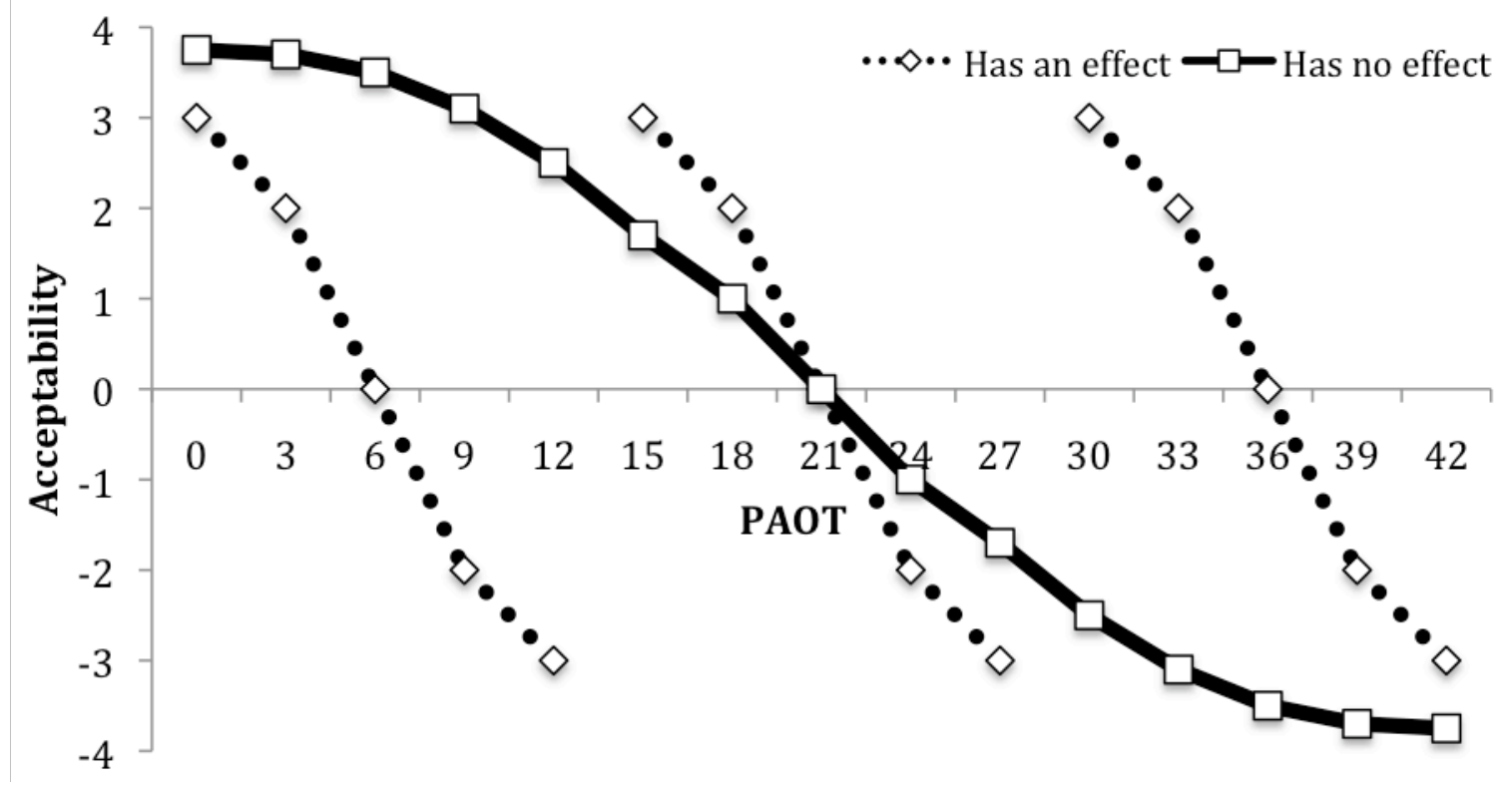

Figure 1-4. Hypothetical acceptability curves illustrating the potential effect of range on acceptability ratings. 


\section{Conclusion}

The visual based method of outdoor recreation research is a common method utilized to provide managers with evaluative social data. Data from visual based studies are commonly used to inform managers on appropriate use-levels for protected areas. The theory and implementation of visual based studies have a deep theoretical background, but not a long history of rigorous testing. The most comprehensive investigation of the validity of visual based methods, by Manning and Freimund (2004), did not go deep enough. Two potential flaws in the visual based method, order effect and range effect biases, were described which may undermine the validity of results from visual based studies.

The following chapters will investigate in detail the two potential problems with visual based methods attributed to photograph presentation order and PAOT range effects. Chapter II focuses on the issue of order effect bias, while Chapter III focuses on the issue of range effect bias. Chapter IV synthesizes the conclusions of Chapters II and III and presents a set of principles that can be applied to avoid the potential problems uncovered by the investigations of order and range effect. 


\section{CHAPTER II}

\section{An Investigation of Photograph Presentation Order Effects in Visual Based Outdoor Recreation Research}

\section{Introduction}

The purpose of this chapter is to investigate a potential bias known as order effect, related to the presentation order of photographs in the visual method. More specifically, this chapter investigates the effect of photograph presentation order on acceptability ratings assigned to photographs when using the visual method. This investigation of presentation order expanded on the Manning et al. (2002) approach (evaluating photo acceptability ratings for sequential and reverse sequential photo presentation orders). A more rigorous investigation of photograph order-effect was conducted, which utilized pseudorandomized photo presentation orders. Data for this investigation were gathered from respondents in a lab environment. Six photograph presentation orders were utilized and evaluation trends were compared through statistical analyses. Linear models were generated testing the relationship between acceptability and multiple predictor variables (PAOT, photo presentation order, and respondent idiosyncrasies). 


\section{Methods}

Participants

The effect of photo presentation order on photo acceptability ratings was tested in a lab setting for a total of 187 undergraduate students from Colorado State University. Students participated in this project as partial fulfillment of a research requirement in their Introductory Psychology course in the spring of 2009. Students were randomly assigned to one of the six different photo presentation order groups (described in the Procedure section).

\section{Apparatus}

The research procedure was intended to replicate the protocol of previous research using the visual method, with the exception that this project was conducted in a lab setting. The experiment took place in a room measuring $18 \mathrm{x}$ $18 \mathrm{ft}(5.5 \mathrm{~m} \times 5.5 \mathrm{~m})$ with walls painted black to minimize reflection. Participants were run through the experimental procedures in groups of four and were seated $10 \mathrm{ft}(3 \mathrm{~m})$ away from a $6 \times 6 \mathrm{ft}(1.8 \mathrm{~m} \times 1.8 \mathrm{~m})$ screen. Photographs were presented on the full screen via computer projector.

\section{Procedure}

Participants were told they would be rating a series of photographs of recreation scenes from Rocky Mountain National Park based on the acceptability of the number of people depicted in the photographs. Participants were shown a series of five digitally edited photographs depicting a trail segment in Rocky Mountain National Park. The numbers of people in each of the five photographs 
were digitally manipulated so that $0,4,8,12$, or 16 people appeared in each photo (Figure 2-1). Photos were shown to respondents one at a time. When shown each photo, respondents were given 20 seconds to rate on a scale ranging from -4 (very unacceptable) to +4 (very acceptable) how acceptable they found the photo regarding the PAOT depicted in the photograph (Figure 2-2). 


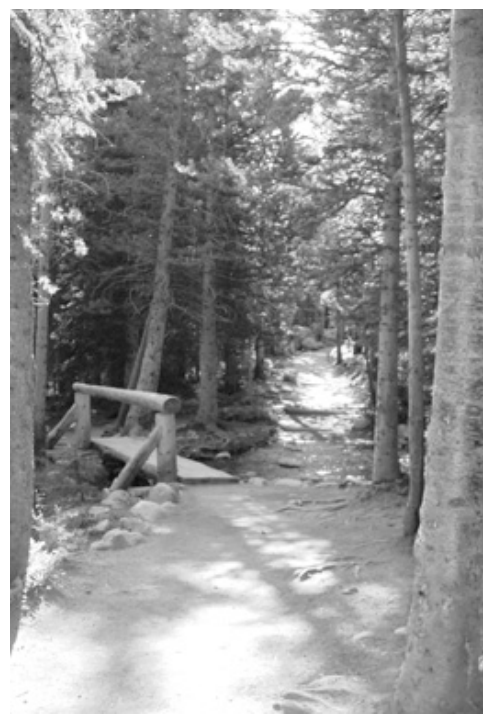

Photo 1

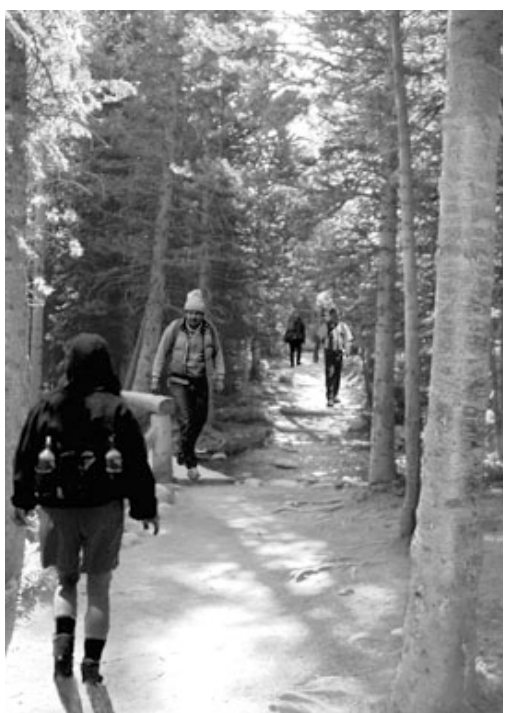

Photo 2

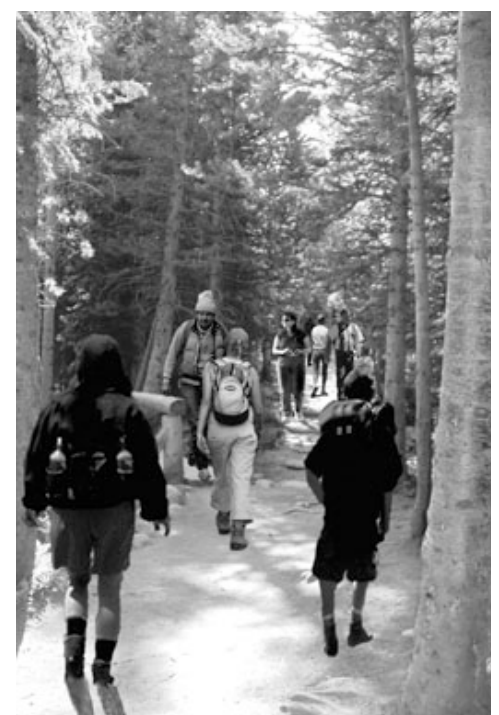

Photo 3

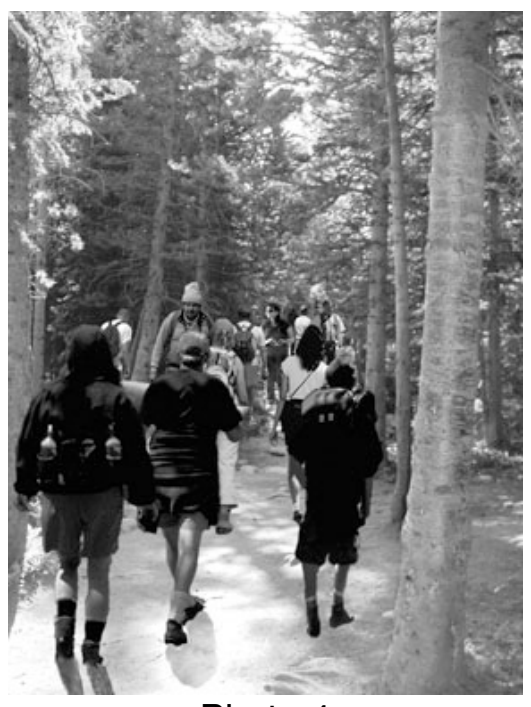

Photo 4

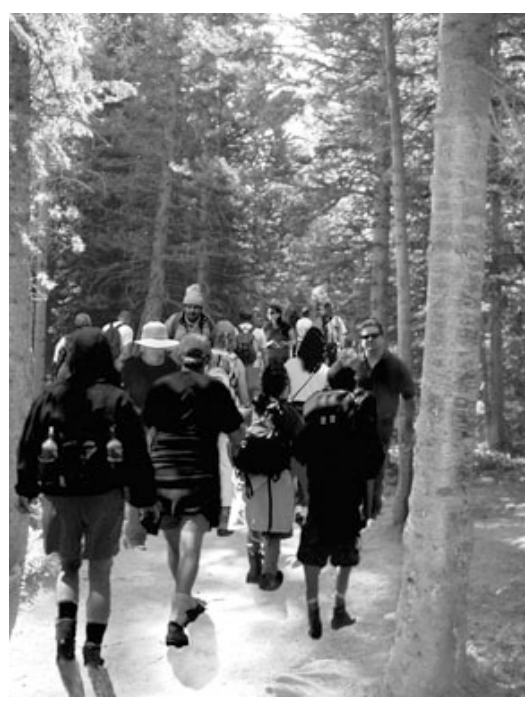

Photo 5

Figure 2-1. Digitally edited photographs of the Glacier Gorge trail in Rocky Mountain National Park. 
We would like to know how many other people you think you could see at one time without feeling too crowded. To help us judge this, please rate each of the photographs by indicating how acceptable you find it based on the number of people in the photo.

\begin{tabular}{lccccccccc} 
& \multicolumn{3}{c}{ Very } & \multicolumn{1}{c}{ Neither } & \multicolumn{3}{c}{$\begin{array}{c}\text { Very } \\
\text { Unacceptable }\end{array}$} \\
\hline $1^{\text {st }}$ Photo & -4 & -3 & -2 & -1 & 0 & +1 & +2 & +3 & +4 \\
$2^{\text {nd }}$ Photo & -4 & -3 & -2 & -1 & 0 & +1 & +2 & +3 & +4 \\
$3^{\text {rd }}$ Photo & -4 & -3 & -2 & -1 & 0 & +1 & +2 & +3 & +4 \\
$4^{\text {th }}$ Photo & -4 & -3 & -2 & -1 & 0 & +1 & +2 & +3 & +4 \\
$5^{\text {th }}$ Photo & -4 & -3 & -2 & -1 & 0 & +1 & +2 & +3 & +4 \\
\hline
\end{tabular}

Figure 2-2. Question wording and format.

Photograph presentation order was experimentally controlled to present photographs in one of six different presentation orders (Table 2-1). As seen in Table 2-1, there are two ordered sequences, a forward and a reverse, and four pseudo-random sequences. The four pseudo-random photo presentation sequences were created to place disparate photos next to one another in the sequence (e.g., photo 1 next to photo 5 , photo 2 next to photo 4 , etc). 


\section{Table 2-1}

Summary of Photo Presentation Orders and Sequences

\begin{tabular}{lc}
\hline Photo order & Photo presentation sequence \\
\hline 1 & $1,2,3,4,5$ \\
2 & $2,3,1,5,4$ \\
3 & $3,5,4,1,2$ \\
4 & $4,1,5,3,2$ \\
5 & $5,1,2,4,3$ \\
6 & $5,4,3,2,1$ \\
\hline
\end{tabular}

Analysis Strategy

Data were analyzed using the statistical software "R". The first step was to summarize the data based on typical procedures from previous visual based studies. A summary of acceptability curve characteristics was generated from each photograph presentation order and mean evaluations, and were compared using one-way analyses of variance (ANOVAs). However, in order to delve deeper into the effect of photograph presentation order on overall evaluative trends, other analyses were utilized.

Non-Monotonic Value Summary

Previous literature related to acceptability of PAOT depicted in photographs (see Chapter I) suggests there is a monotonic relationship between PAOT and acceptability (Manning, 2007). A monotonic relationship between acceptability and PAOT means that acceptability ratings decline with evaluations of successive photos, or in other words, as PAOT increases. Non-monotonic 
values were summarized for each photograph presentation order, because the frequency of non-monotonic values may tell us something about the effect of presentation order. For example, if non-monotonic values occurred more frequently in pseudo-random photograph presentation orders, this may suggest that photograph presentation orders affected photograph acceptability ratings.

\section{Linear Models}

Three linear models were generated predicting acceptability ratings. Model 1 used $\log _{10}$ PAOT to predict photograph acceptability ratings. Model 2 predicted photograph acceptability ratings from $\log _{10}$ PAOT and photograph presentation order. Model 3 predicted photograph acceptability ratings from $\log _{10}$ PAOT, photograph presentation order, and the individual idiosyncrasies of individuals (calculated using Tukey's Median Polish function, Mosteller \& Tukey, 1977). Further explanation of the variables used in the linear models is detail in the subsequent sections.

\section{Results}

The respondent sample included 187 subjects with 92 females and 95 males, and the average age was 20 years old $(M=19.68, S D=1.96)$. The 187 subjects were divided into six photo presentation order groups with between 29 and 33 subjects per group. Table $2-2$ displays a summary of the acceptability curve characteristics, and Table 2-3 displays a summary of means for each photograph by photo presentation order. Based on the results from Table 2-2 and Table 2-3, there appears to be no significant effect of presentation order on 
photograph acceptability ratings. However, a closer look at the data tells a different story.

Table 2-2

Summary of Acceptability Curve Characteristics for Each Presentation Order

\begin{tabular}{lccc|c}
\hline & \multicolumn{3}{c|}{ PAOT } & Acceptability \\
\cline { 2 - 5 } Order & $\begin{array}{c}\text { Preferred or } \\
\text { Optimal } \\
\text { Condition }\end{array}$ & $\begin{array}{c}\text { Range of } \\
\text { Acceptable } \\
\text { Conditions }\end{array}$ & $\begin{array}{c}\text { Minimum } \\
\text { Acceptable } \\
\text { Condition }\end{array}$ & Norm Intensity \\
\hline 1 & 0 & 0 to 8 & 8 & 5.9 \\
2 & 0 & 0 to 7 & 7 & 5.9 \\
3 & 0 & 0 to 7 & 7 & 5.7 \\
4 & 0 & 0 to 7 & 7 & 6.5 \\
5 & 0 & 0 to 7 & 7 & 5.8 \\
6 & 0 & 0 to 9 & 9 & 5.4 \\
\hline
\end{tabular}

Table 2-3

Summary of Means and ANOVA Results of Acceptability Ratings for Each Photo Order by Photo Number

\begin{tabular}{|c|c|c|c|c|c|c|c|c|c|c|}
\hline Order & \multicolumn{2}{|c|}{ Photo 1} & \multicolumn{2}{|c|}{ Photo 2} & \multicolumn{2}{|c|}{ Photo 3} & \multicolumn{2}{|c|}{ Photo 4} & \multicolumn{2}{|c|}{ Photo 5} \\
\hline 1 & \multicolumn{2}{|c|}{3.3} & \multicolumn{2}{|c|}{1.7} & \multicolumn{2}{|c|}{0.1} & \multicolumn{2}{|c|}{-1.4} & \multicolumn{2}{|c|}{-2.6} \\
\hline 2 & \multicolumn{2}{|c|}{3.5} & \multicolumn{2}{|c|}{1.0} & \multicolumn{2}{|c|}{-0.4} & \multicolumn{2}{|c|}{-1.6} & \multicolumn{2}{|c|}{-2.4} \\
\hline 3 & \multicolumn{2}{|c|}{3.3} & \multicolumn{2}{|c|}{1.8} & \multicolumn{2}{|c|}{-0.3} & \multicolumn{2}{|c|}{-1.2} & \multicolumn{2}{|c|}{-2.4} \\
\hline 4 & \multicolumn{2}{|c|}{3.5} & \multicolumn{2}{|c|}{1.0} & \multicolumn{2}{|c|}{-0.3} & \multicolumn{2}{|c|}{-1.4} & \multicolumn{2}{|c|}{-3.0} \\
\hline 5 & \multicolumn{2}{|c|}{3.4} & \multicolumn{2}{|c|}{1.3} & \multicolumn{2}{|c|}{-0.3} & \multicolumn{2}{|c|}{-1.7} & \multicolumn{2}{|c|}{-2.4} \\
\hline \multirow[t]{3}{*}{6} & \multicolumn{2}{|c|}{3.6} & \multicolumn{2}{|c|}{2.1} & \multicolumn{2}{|c|}{0.5} & \multicolumn{2}{|c|}{-1.0} & \multicolumn{2}{|c|}{-1.8} \\
\hline & $F$ & $\eta^{2}$ & $F$ & $\eta^{2}$ & $F$ & $\eta^{2}$ & $F$ & $\eta^{2}$ & $F$ & $\eta^{2}$ \\
\hline & 0.32 & .01 & 1.84 & .01 & 0.85 & .03 & 0.49 & .03 & 1.09 & .01 \\
\hline
\end{tabular}




\section{Non-Monotonic Values}

Figure 2-3 displays the raw data comparing photograph number (PAOT increases with each photo) to photograph acceptability. In essence, Figure 2-2 displays each individual's acceptability curve. Based on this figure it is difficult to see the expected relationship between PAOT and acceptability. Of particular interest are the acceptability curves exhibiting a non-monotonic relationship between acceptability and photo number.

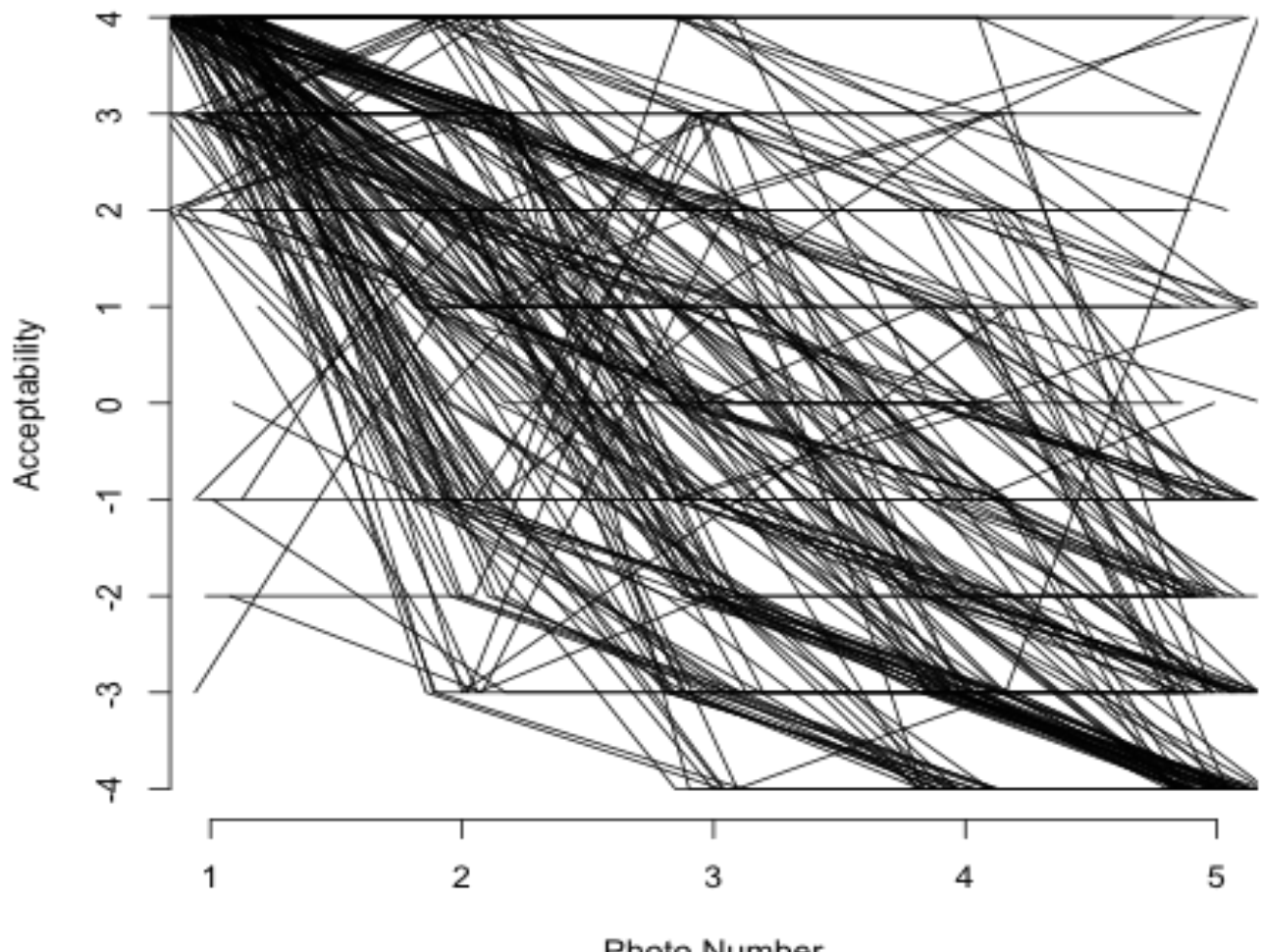

Photo Number

Figure 2-3: Summary of each individual's acceptability curve.

Table 2-4 displays a summary of the non-monotonic values for each photo presentation order by photo segment. Given the theoretical relationship between acceptability and PAOT, all values in this table should be 0 , with the exception of those individuals that prefer a few people rather than 0 people. There should be a 
completely monotonic relationship between acceptability and photo number for photographs depicting $>0$ people. Table 2-4 indicates that non-monotonic values occurred 1 time for non-random sequences (Order 1 and Order 6), and 36 times for pseudo-random sequences (Orders $2-5)$. In other words, non-monotonic values occurred less than $1 \%$ of the time for non-random photograph presentation orders, and between $5 \%$ and $11 \%$ of the time for pseudo-random sequences.

Table 2-4

Summary of Non-Monotonic Values by Photo Presentation Order

\begin{tabular}{lcccccc}
\hline & \multicolumn{7}{c}{ Non-Monotonic Values by Photo Segment } & & \\
\cline { 2 - 5 } Order & 1 to 2 & 2 to 3 & 3 to 4 & 4 to 5 & Total & Percent \\
\hline 1 & 1 & 0 & 0 & 0 & 1 & $<1 \%$ \\
2 & 3 & 5 & 4 & 1 & 13 & $11 \%$ \\
3 & 3 & 2 & 2 & 2 & 9 & $7 \%$ \\
4 & 1 & 3 & 1 & 1 & 6 & $5 \%$ \\
5 & 3 & 2 & 2 & 1 & 8 & $7 \%$ \\
6 & 0 & 0 & 0 & 0 & 0 & $0 \%$ \\
\hline
\end{tabular}

${ }^{1}$ Five photos results in four photograph segments. For example, in the "1 to 2" segment, respondents evaluated photo 2 as more acceptable than photo 1 , etc.

Table 2-4 also indicates that 36 out of 37 of the non-monotonic values occurred in the random photo presentation orders, indicating that $97 \%$ of all the non-monotonic values occurred when photographs were presented pseudorandomly. The odds of 36 out of 37 non-monotonic occurring in the random order sequences (Orders $2-5$ ) by chance alone are about 1 in 325,000 . That is to say, 
there is little chance that the disproportionate occurrence of non-monotonic values in the pseudo-random photograph presentation orders is due to chance alone. The summary non-monotonic values suggest the data are more complex than the summary of acceptability curves and means implied.

\section{Linear Analyses}

$\log _{10}$ of PAOT was used in the linear analyses and not simply PAOT, because the relationship between PAOT and acceptability is not assumed to be linear. The relationship between acceptability and PAOT is not linear because it is much easier to notice a change in PAOT when PAOT is low than when PAOT is high (Manning, Lime, Freimund, \& Pitt, 1996). In other words, the effect of a 4 PAOT increase from 4 PAOT to 8 PAOT is not the same as the effect of a 4 PAOT increase from 12 PAOT to 16 PAOT. One drawback in using $\log _{10}$ PAOT is that acceptability ratings from Photograph 1 (0 PAOT) must be eliminated from the analysis because the $\log _{10}$ of 0 is undefined. However, there is some theoretical justification for removing Photograph 1.

One reason for eliminating Photograph 1 from the analyses has to do with the consistently high acceptability ratings with 0 PAOT. According to Figure 2-4, Photograph 1 had a median acceptability rating of 4 , the highest possible, which means it is not particularly informative when looking for differences. There were also no people in the photograph, which made direct comparisons between acceptability of Photograph 1 and the photographs with people in them (Photos 2 -5) problematic. For example, respondents were asked to evaluate the 
acceptability of the number of people depicted in the photo, but there were no people depicted in Photo 1.

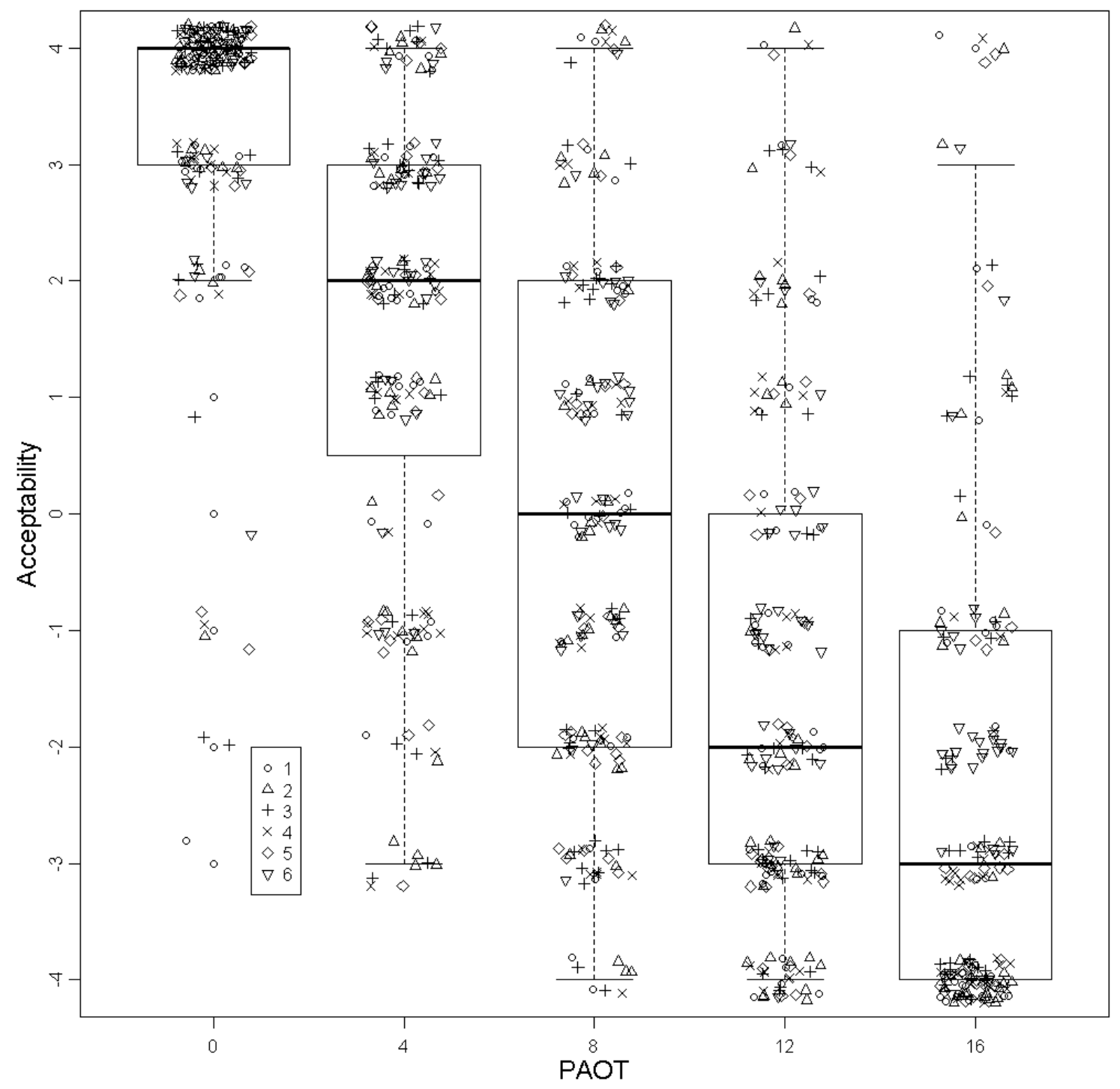

Figure 2-4: Raw Data Box Plot 


\section{Model 1 - Photograph Acceptability and Photograph Number}

The first linear model, Model 1 , used $\log _{10}$ of PAOT to predict photograph acceptability using univariate regression. Model 1 significantly predicted photograph acceptability ratings, $F(1,736)=1115.52, p$-value $<.001$, where $\log _{10}$ of PAOT explained approximately $33 \%$ of the variance in photo acceptability ratings. Model 1 should form the basis by which to compare subsequent models because theoretically photo acceptability ratings should be a function of PAOT. Model 2 - Photograph Acceptability and Photograph Number with Photo Order

Model 2 used photograph presentation order to predict photograph acceptability ratings while controlling for $\log _{10}$ PAOT by using a family of univariate linear regressions. Results indicated that photo order significantly predicted photo acceptability ratings, $F(5,736)=2.99$, $p$-value $=.01$, when controlling for $\log _{10}$ PAOT. Results indicate photograph presentation order predicted less than $1 \%$ of the variance in photograph acceptability ratings. Model 3 - Photograph Acceptability and Photograph Number with Presentation Order and Individual Idiosyncrasy

Model 3 used a family of bivariate regressions to predict photograph acceptability ratings while controlling for the effect of $\log _{10}$ PAOT and differences in individual idiosyncrasies of responses. Model 3 incorporated a variable, Tukey's Median Polish values, to account for the individual idiosyncrasies with respect to acceptability ratings. Individual idiosyncrasy was explored as a control variable, because it was suspected of masking the effects of photograph 
presentation order on acceptability ratings. Tukey's median polish row values were used to represent individual idiosyncrasies (Mosteller \& Tukey, 1977). The median polish operation creates a median polish value for each row and column in the data table through an iterative process of subtracting the row and column median from each value in a data table. The process continues until each value in the table is 0 , and the medians from each iteration are summed resulting in row effect values and column effect values.

When applied to the data used in this paper, median polish values were obtained for each individual (row effect) and each photograph (column effect). The median polish values for each individual represented the individual differences in photograph evaluation without using an exorbitant number of degrees of freedom. The median polish operation did not use very many degrees of freedom because the median polish operation grouped people with like evaluation trends.

Accounting for individual differences allowed Model 3 to better predict acceptability scores than Model 2 by accounting for the variance unexplained by photo number and photo presentation order. Results from Model $3, F(5,736)=$ 16.49, $p$-value $<.001$, indicated photograph presentation order predicted about $2 \%$ of the variance in photograph acceptability ratings.

A post-hoc test of Model 3 investigating the individual effects of the various photograph presentation orders determined there were significant differences for Order 2, coefficient $=-1.0, t=6.80, p$-value $<.001$, and Order 5, 
coefficient $=-0.7, t=4.88, p$-value $<.001$. These results indicate that for Photographs 2 through 5, Order 2 values are estimated to be 1.0 units of acceptability lower than Order 1 values, and Order 5 values are estimated to be 0.7 units of acceptability lower than Order 1 values. Comparatively, the coefficients related to $\log _{10}$ PAOT range from -1.34 to -1.94 . Comparing the coefficients for $\log _{10}$ PAOT and photograph presentation order indicates that the effect of photograph presentation order on acceptability ratings is less than the effect of PAOT on acceptability ratings (Figure 2-5).

Coefficient values are displayed for each photograph by presentation order in Figure 2-5 as a graphical representation of acceptability curves. Figure 2-4 shows that acceptability ratings from Order 2 and Order 5 are shifted down about one unit of acceptability as a result of the effect of presentation order.

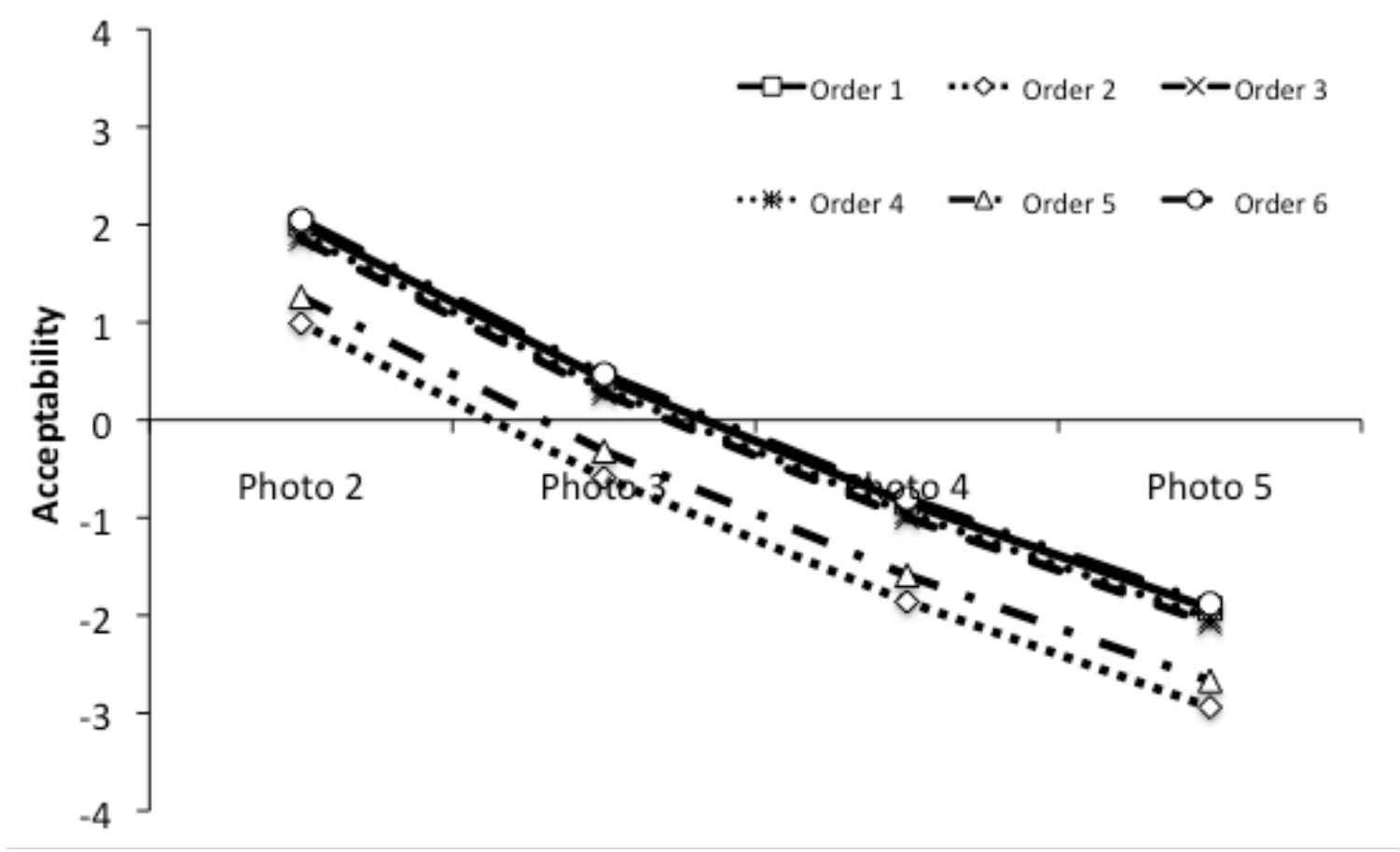

Figure 2-5: Plot of coefficient values as acceptability curves. 
Results from Model 3 are depicted in Figure 2-6. This figure shows how the hypothesized monotonic relationship between PAOT and acceptability becomes much more pronounced when the model accounts for the effects of PAOT, individual differences, and presentation order. This figure summarizes the ability of Model 3 to account for variations in acceptability ratings, by displaying predicted acceptability ratings. 

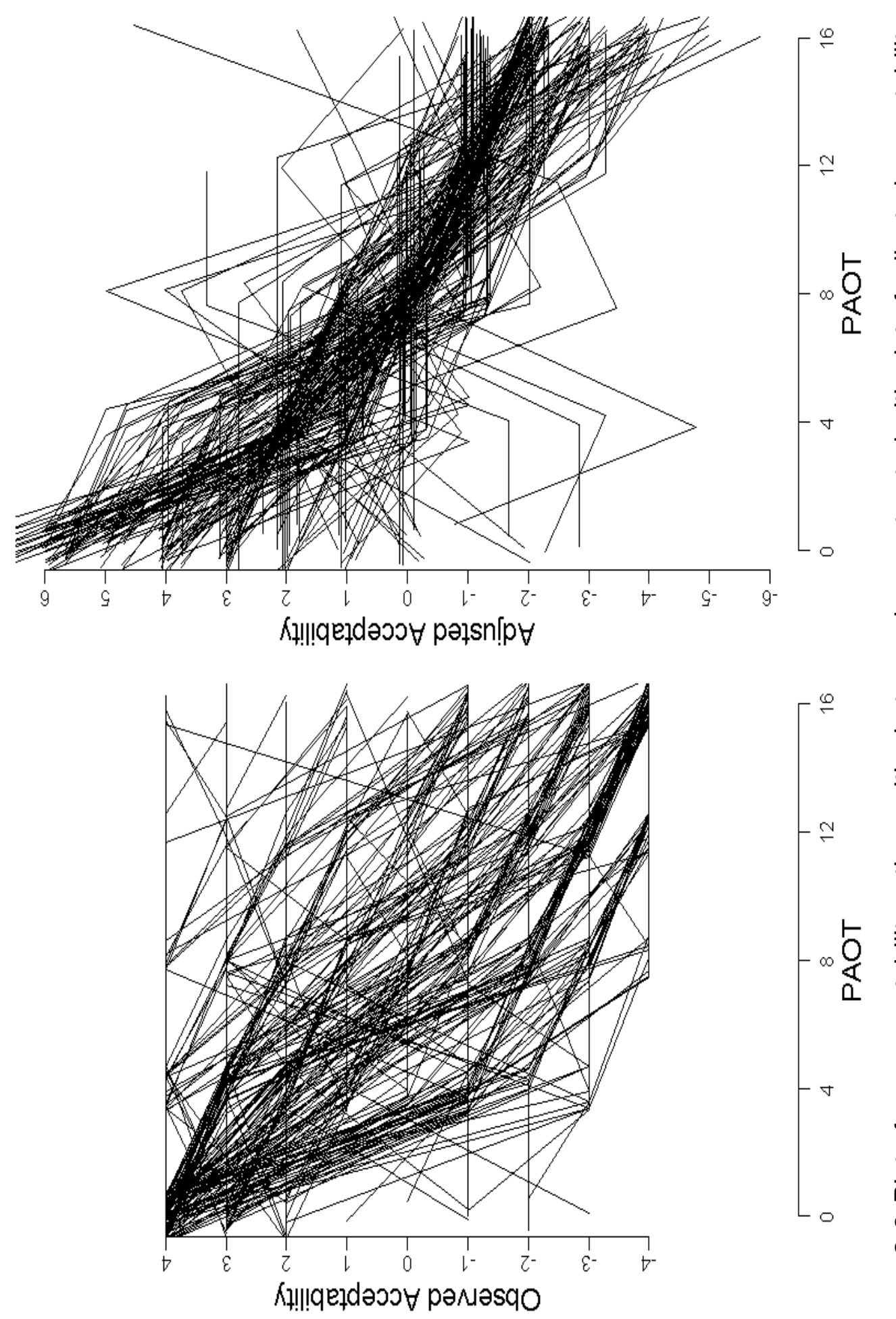

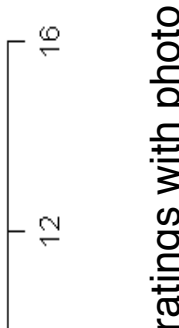

$\leftarrow$
$-\infty$
$\frac{1}{\square}$

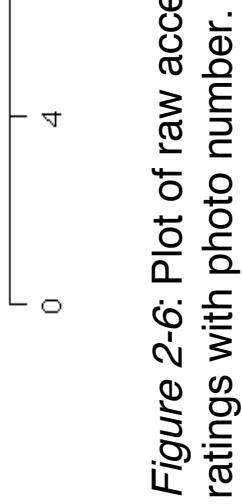




\section{Discussion}

The purpose of this chapter was to investigate the effect photograph presentation order had on photograph acceptability ratings in visual-based outdoor recreation research methods. According to the one-way ANOVAs, presentation order did not significantly affect photograph acceptability ratings (Table 2-3). These results implied that acceptability ratings obtained from multiple photograph presentation orders were not significantly different. However, the distribution of non-monotonic values and linear models tell a different story.

\section{Non-Monotonic Values}

Results from Table 2-4 indicated a disproportionate amount of nonmonotonic values occurred in the random photograph presentation orders. As stated earlier, the chances of 36 out of 37 non-monotonic values occurring in the random sequences is about 1 in 350,000 . This low level of probability ( $p$-value $<$ .001) suggests that while the mean acceptability ratings of each photo may not be significantly different, there may be differences in the overall evaluation trends associated with each order.

\section{Linear Models}

Linear models showed that presentation order significantly affected photograph acceptability ratings. A one-way ANOVA was not able to detect differences in acceptability ratings, because the one-way ANOVA compared means within a segment of $1 / 6$ of the data, based on each photo presentation order. Comparatively, the linear models used the entire dataset to look for 
relationships between acceptability and the predictors. In other words, the linear models investigated differences in evaluation trends of the entire set of photographs, while the one-way ANOVA compared a segmented set of means.

Results from the final linear model (Model 3) indicated that when the effects of PAOT and individual differences are controlled, photograph presentation order explains about $2 \%$ of the variance in acceptability ratings for Photos $2-5$. Results also indicated there were only significant differences for presentation Order 2 and Order 5, where the coefficients were -1.0 and -0.7. A 1.0 coefficient corresponds to a "shift" in the acceptability curve down one unit of acceptability (Figure 2-5). These results are unique because they suggest that the evaluation of photographs in terms of the acceptability of people at one time is influenced by the order in which photographs were presented.

\section{Limitations}

One limitation of the final linear model concerns the effect size of the photograph presentation order variable. In the final linear model, photograph presentation order was only able to explain $2 \%$ of the variance in acceptability ratings. While the effect of presentation order on acceptability ratings is significant, it is not large. The number of people depicted in the photos $\left(\log _{10}\right.$ of PAOT) was still a much better predictor of acceptability than presentation order.

This investigation of photograph presentation order was also conducted in a lab setting. Visual methods are generally used in the field where the respondent is assumed to have firsthand knowledge of the site pictured in the 
photographs. This assumption was not met in this study, and it is unknown what effect this may have had on the results.

\section{Conclusion}

There were significant differences in acceptability ratings of the photographs when multiple photograph presentation orders were used, and to some extent those differences were a function of photograph presentation order. However, the question of whether or not those differences are large enough to matter may not be for the researcher to decide. When reviewing the results of this investigation of order effect, it is important to keep in mind that social evaluative standards do not exist in a vacuum. Management decisions regarding appropriate use levels are never exclusively derived from the results of visitor questionnaires. Management decisions are the product of social, environmental, and managerial variables, and are largely directed by management objectives (Manning, 2007). Even with the existence of order effects, visual based data might still be useful for managers.

Moore (2002) contends "the rotation of questions and identification of these effects remind us of how variable are the measures of such opinions" (p. 89). Use of pseudo-random photograph presentation orders have the potential to yield more robust measures of evaluative standards by controlling for the bias of order effect. However, order effect is not the only potential bias of the visual method. The next chapter investigates how PAOT range may affect acceptability ratings by presenting respondents with multiple PAOT ranges and comparing 
acceptability rating results in similar fashion as was used to investigate order effect bias. Furthermore, results from the investigation of order effect bias will be combined with an investigation of range effect bias in Chapter IV to recommend revisions of visual based methods of outdoor recreation research. 


\section{CHAPTER III}

\section{An Investigation of Photograph Range Effects in Visual Based Outdoor \\ Recreation Research}

\section{Introduction}

This chapter continues the investigation of visual methods by testing a bias referred to as range effect. For the purposes of this dissertation, "PAOT range" is a catch-all term referring to the following characteristics of the PAOT range: difference between the maximum and minimum PAOT depicted in a group of photographs, incremental increase of PAOT with each photograph, and starting and ending PAOT. Range effect bias is the potential effect various PAOT ranges of photograph sets may have on acceptability ratings assigned to photographs used in the visual method. As previously discussed, photographs are commonly used to inform evaluative use-level standards for recreation areas. Depending on the PAOT range chosen for a set of photographs, results from visual based studies may suggest multiple use-level standards.

\section{Purpose}

The purpose of this chapter was to investigate the issue of range effect in visual based methods. Photograph sets depicting various PAOT ranges were presented to lab respondents, and respondents rated photographs in terms of acceptability of depicted PAOT. The effect of PAOT range on acceptability ratings was investigated through the creation of linear models predicting acceptability ratings of photographs. Various models were created using $\log _{10}$ 
PAOT, range group, and photograph sequence number (i.e., whether a photograph was presented $1^{\text {st }}, 2^{\text {nd }}, 3^{\text {rd }}, 4^{\text {th }}$, or $\left.5^{\text {th }}\right)$. Models were compared based on statistical characteristics such as degrees of freedom, significance level, and amount of variance explained.

\section{Methods}

\section{Participants}

The effect of PAOT range on photo acceptability ratings was tested in a lab setting for a total of 305 undergraduate students from Colorado State University. Students participated in the project as partial fulfillment of a research requirement in their Introductory Psychology course in the spring of 2010.

Students were randomly assigned to one of seven different PAOT range groups (described in the Procedure section).

\section{Apparatus}

The research procedure was intended to replicate the protocol of previous research using the visual method, with the exception that this project was conducted in a lab setting. The experiment took place in a room measuring $18 \mathrm{x}$ $18 \mathrm{ft}(5.5 \mathrm{~m} \times 5.5 \mathrm{~m})$ with walls painted black to minimize reflection. Participants were run through the experimental procedures in groups of four and were seated $10 \mathrm{ft}(3 \mathrm{~m})$ away from a $6 \times 6 \mathrm{ft}(1.8 \mathrm{~m} \times 1.8 \mathrm{~m})$ screen. Photographs were presented on the full screen via computer projector. 


\section{Procedure}

Respondents were shown a series of five digitally edited photographs depicting a popular recreation destination in Rocky Mountain National Park where the numbers of people in each photo were digitally manipulated so that between 0 and 42 people appeared in each photo. The five photos shown to respondents were part of a larger series of 15 photographs ranging in PAOT from 0 to 42 . In the 15-photograph set, PAOT increased by 3 with each subsequent photograph (Figure 3-1). The PAOT range for each five-photograph series was experimentally controlled to represent one of seven different PAOT ranges (Table 3-1). 


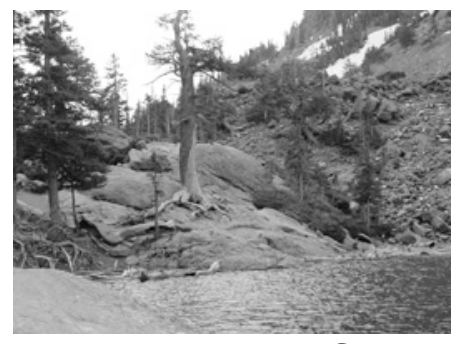

Photo 1 (0 PAOT)

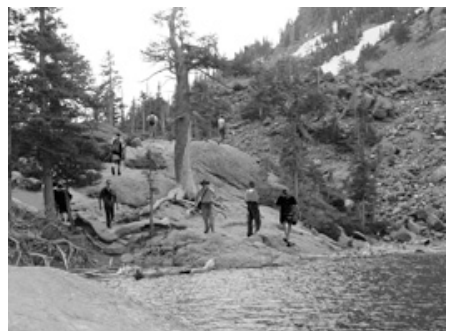

Photo 4 (9 PAOT)

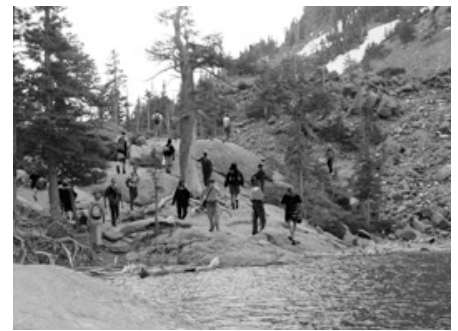

Photo 7 (18 PAOT)

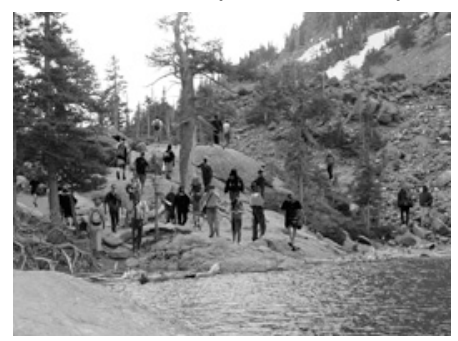

Photo 10 (27 PAOT)

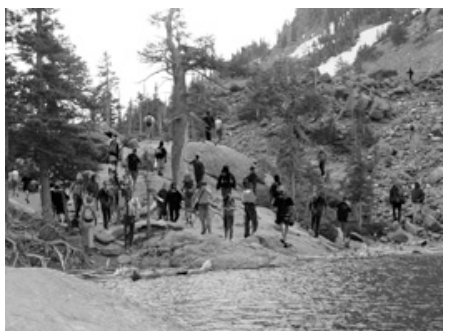

Photo 13 (36 PAOT)

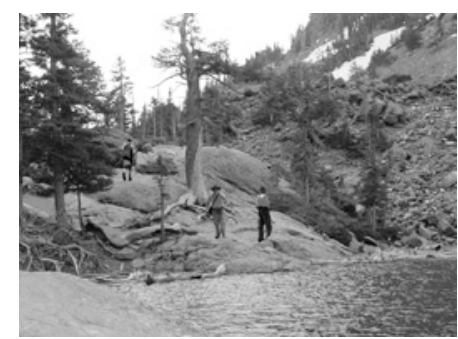

Photo 2 (3 PAOT)

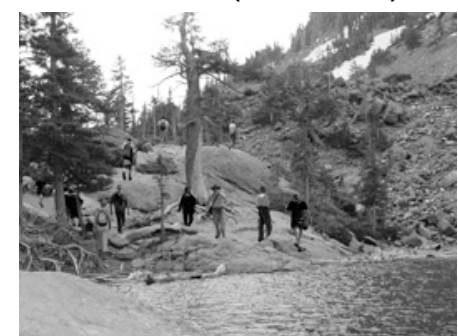

Photo 5 (12 PAOT)

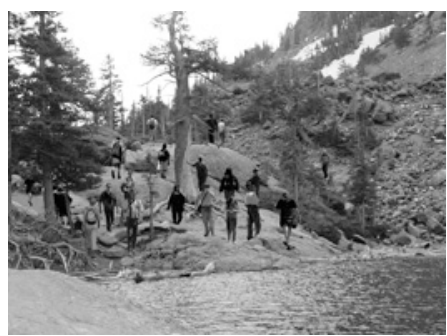

Photo 8 (21 PAOT)

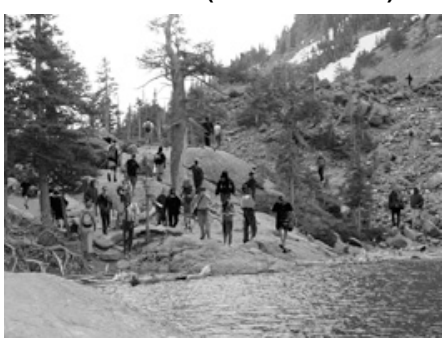

Photo 11 (30 PAOT)

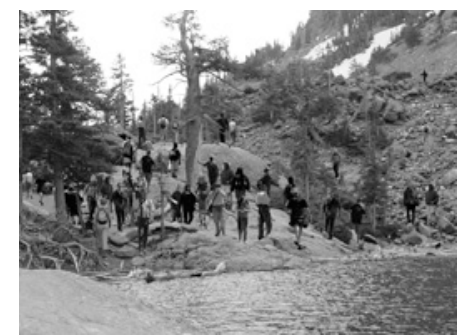

Photo 14 (39 PAOT)

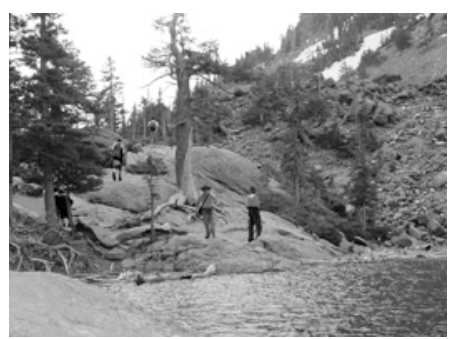

Photo 3 (6 PAOT)

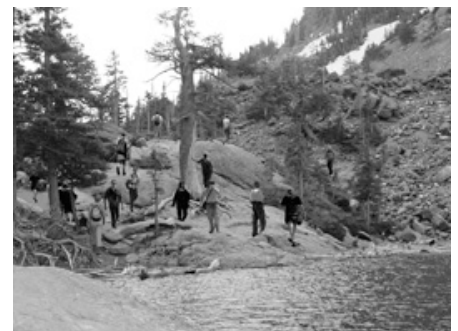

Photo 6 (15 PAOT)

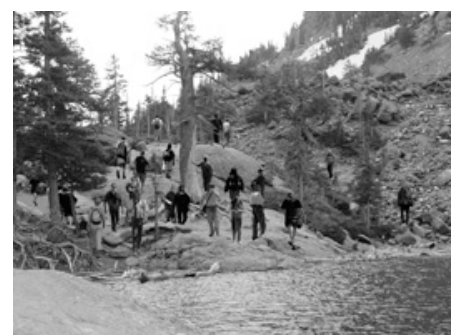

Photo 9 (24 PAOT)

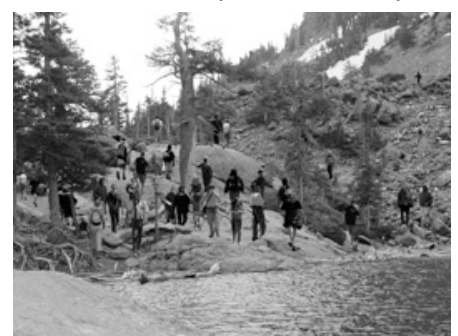

Photo 12 (33 PAOT)

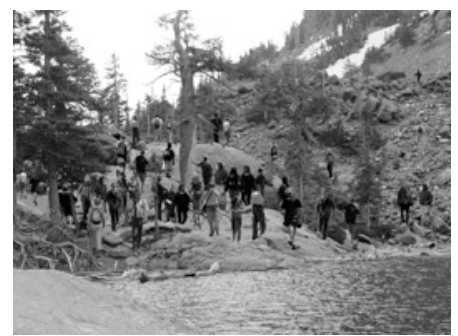

Photo 15 (42 PAOT)

Figure 3-1. Digitally edited photographs of Emerald Lake in Rocky Mountain National Park. 
We would like to know how many other people you think you could see at one time without feeling too crowded. To help us judge this, please rate each of the photographs by indicating how acceptable you find it based on the number of people in the photo.

\begin{tabular}{lccccccccc} 
& \multicolumn{3}{c}{ Very } & \multicolumn{1}{c}{ Neither } & \multicolumn{3}{c}{$\begin{array}{c}\text { Very } \\
\text { Unacceptable }\end{array}$} \\
\hline $1^{\text {st }}$ Photo & -4 & -3 & -2 & -1 & 0 & +1 & +2 & +3 & +4 \\
$2^{\text {nd }}$ Photo & -4 & -3 & -2 & -1 & 0 & +1 & +2 & +3 & +4 \\
$3^{\text {rd }}$ Photo & -4 & -3 & -2 & -1 & 0 & +1 & +2 & +3 & +4 \\
$4^{\text {th }}$ Photo & -4 & -3 & -2 & -1 & 0 & +1 & +2 & +3 & +4 \\
$5^{\text {th }}$ Photo & -4 & -3 & -2 & -1 & 0 & +1 & +2 & +3 & +4 \\
\hline
\end{tabular}

Figure 3-2. Question wording and format.

Table 3-1

\begin{tabular}{lcc}
\multicolumn{3}{l}{$\begin{array}{l}\text { Summary of PAOT Range Groups and Corresponding Photo Sequences and } \\
\text { PAOT Ranges }\end{array}$} \\
\hline Range Group & Photograph Number & PAOT Range \\
\hline 1 & $1,2,3,4,5$ & $0-12$ \\
2 & $6,7,8,9,10$ & $15-27$ \\
3 & $11,12,13,14,15$ & $30-42$ \\
4 & $1,4,7,10,13$ & $0-36$ \\
5 & $3,6,9,12,15$ & $6-42$ \\
6 & $1,3,5,7,9$ & $0-24$ \\
7 & $7,9,11,13,15$ & $18-42$ \\
\hline
\end{tabular}




\section{Analyses}

Data were analyzed using the statistical software "R". In order to deeply explore the relationship between PAOT and acceptability in the context of PAOT range, linear models were generated. Three linear models were produced predicting acceptability ratings. Model 1 predicted acceptability using $\log _{10}$ PAOT. Model 2 predicted acceptability using $\log _{10} \mathrm{PAOT}$, and a categorical variable representing photograph range group (Table 3-1). Finally, Model 3 predicted acceptability using $\log _{10}$ PAOT and photo presentation sequence number.

\section{Results}

The respondent sample included 305 subjects where 190 were females and 115 were males, and the average age was 20 years old $(M=20.02, S D=$ 2.81). The 305 subjects were divided into seven PAOT range groups with 100 subjects for PAOT Range 1, and between 32 and 37 subjects per group for PAOT ranges $2-7$. Table 3-2 displays the mean acceptability ratings for each range group by photo number. 
Table 3-2

Summary of Mean Photo Acceptability Ratings by PAOT Range Group

\begin{tabular}{lccccccc} 
& Range 1 & Range 2 & Range 3 & Range 4 & Range 5 & Range 6 & Range 7 \\
\cline { 2 - 8 } Photo & $M$ & $M$ & $M$ & $M$ & $M$ & $M$ & $M$ \\
\hline 1 & 3.82 & -- & -- & 3.97 & -- & 3.82 & -- \\
2 & 2.83 & -- & -- & -- & -- & -- & -- \\
3 & 1.98 & -- & -- & -- & 2.57 & 2.48 & -- \\
4 & .53 & -- & -- & 1.08 & -- & -- & -- \\
$5^{\star}$ & $-.93^{\mathrm{a}}$ & -- & -- & -- & -- & $.39^{\mathrm{b}}$ & -- \\
6 & -- & .44 & -- & -- & -51 & -- & -- \\
$7^{\star}$ & -- & $-.17^{\mathrm{a}, \mathrm{b}}$ & -- & $-.61^{\mathrm{a}, \mathrm{b}}$ & -- & $-1.39^{\mathrm{a}}$ & $.06^{\mathrm{b}}$ \\
8 & -- & -.61 & -- & -- & -- & -- & -- \\
$9^{*}$ & -- & $-1.17^{\mathrm{a}}$ & -- & -- & $-1.14^{\mathrm{a}}$ & $-2.61^{\mathrm{b}}$ & $-.59^{\mathrm{a}}$ \\
10 & -- & -1.94 & -- & -2.22 & -- & -- & -- \\
11 & -- & -- & -.89 & -- & -- & -- & -1.25 \\
12 & -- & -- & -1.57 & -- & -2.23 & -- & -- \\
13 & -- & -- & -2.03 & -2.89 & -- & -- & -2.16 \\
14 & -- & -- & -2.32 & -- & -- & -- & -- \\
15 & -- & -- & -2.62 & -- & -3.00 & -- & -2.72 \\
\hline
\end{tabular}

*Significant differences exist at $p<.05$

Figure 3-3 displays the raw photo acceptability ratings for each individual. PAOT is located on the x-axis, while acceptability is located on the $y$-axis. Figure 3-3 appears chaotic, but a few trends are observable from these data. For example, a large majority of individuals evaluated 0 PAOT as highly acceptable. There is also a general downward trend (top left to bottom right) in acceptability ratings from 0 PAOT to 42 PAOT. Finally, above 30 PAOT many evaluations are rated as Highly Unacceptable. Given the wide range of responses for any PAOT level, the results from Figure 3-3 suggest that range affected acceptability ratings 
to some degree, but the actual extent cannot be determined from a summary of means or a plot of the raw data.

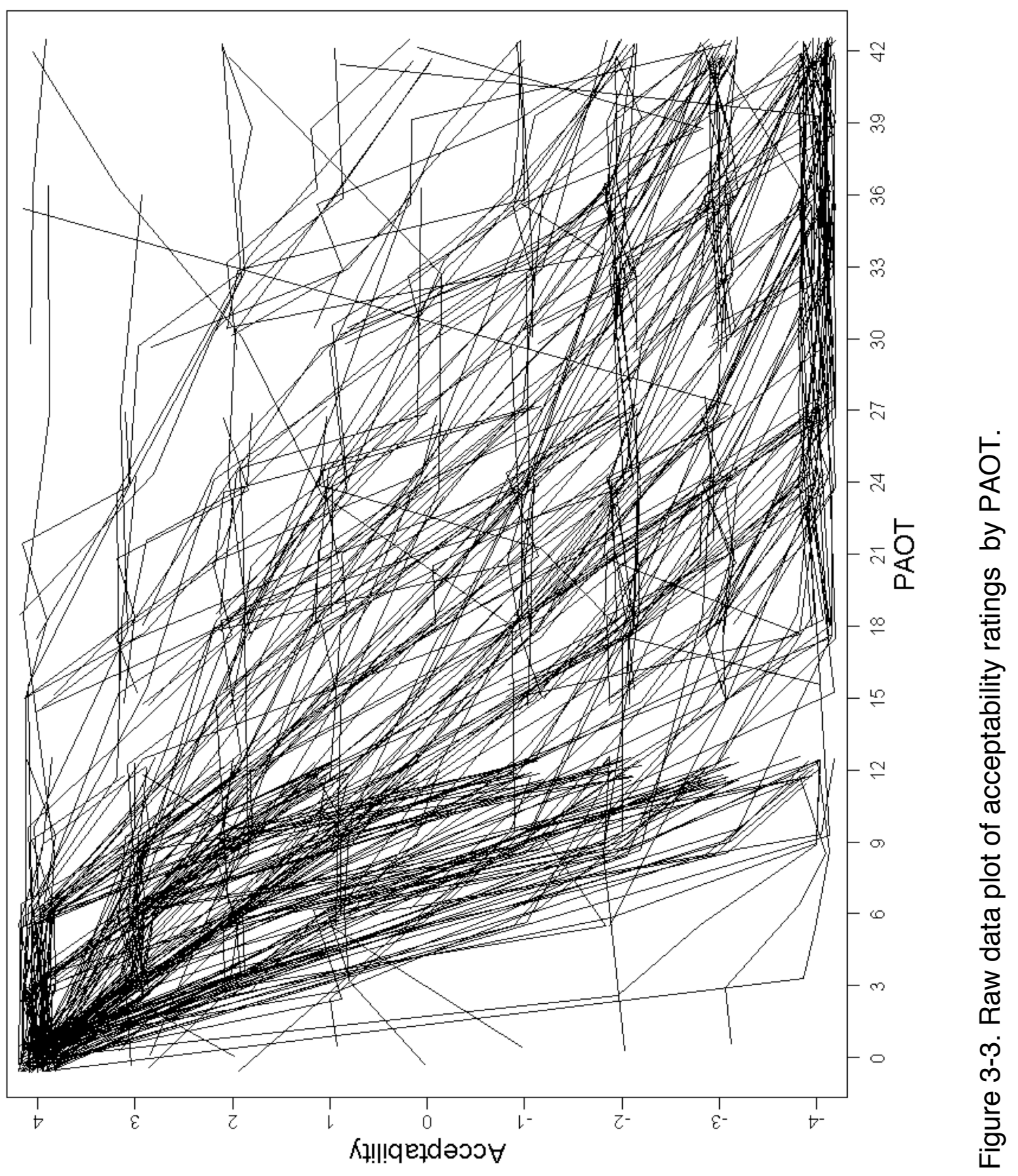




\section{Linear Models}

Linear models were utilized to investigate the extent to which range affected acceptability ratings. The following models compared differences in evaluation trends based on PAOT range. Three models were generated predicting photograph acceptability ratings.

Model 1 - Photograph Acceptability Ratings and $\log _{10}$ of PAOT

Model 1 used $\log _{10}$ PAOT to predict photograph acceptability ratings with the help of a univariate regression. Results from Model 1 indicated the model significantly predicted photograph acceptability ratings, $F(1,1368)=76.85, p$ value $<.001$, where $\log _{10}$ PAOT explained approximately $69 \%$ of the variance in photograph acceptability ratings.

Model 2 - Photograph Acceptability and $\log _{10}$ of PAOT and Range Group

Model 2 predicted acceptability ratings from $\log _{10}$ PAOT and the categorical variable range group (Table 3-1) using a family of univariate linear regressions. Results indicated that range group significantly predicted photograph acceptability ratings, $F(6,1366)=5.59$, $p$-value $<.001$, where range group explained about $5 \%$ of the variance in acceptability ratings.

Model 3 - Photograph Acceptability and $\log _{10}$ of PAOT and Photograph

Sequence Number

Model 3 predicted acceptability from $\log _{10}$ PAOT and photograph presentation sequence number using a family of bivariate regressions. However, the variable of interest in Model 3 was photograph sequence number, and not the 
photograph number corresponding to PAOT. In other words, photograph presentation sequence number categorically referred to the order in which each photograph was presented to the respondent. Each respondent saw five photographs, so this value ranged from 1 to 5 .

Model 3 significantly predicted acceptability, where sequence number, $F(4,1368)=9.13, p$-value $<.001$, predicted about $6 \%$ of the variance in acceptability ratings. Results from a post-hoc test indicated that all sequence numbers significantly predicted acceptability ratings at $p$-value $<.001$. Table 3-3 summarizes the coefficients associated with each sequence number. Table 3-3 indicates that, for a given PAOT level, photographs evaluated $5^{\text {th }}$ were typically 1.61 units of acceptability lower than photographs evaluated $1^{\text {st }}$. Figure $3-4$ graphically displays the effect of photograph presentation sequence on acceptability ratings.

Figure 3-4 suggests that for a given PAOT value, the acceptability rating depends on the $\log _{10}$ PAOT, but whether the PAOT value was at the low end of the range or the high end of the range. Figure 3-4 shows there is a subtractive effect in the number of photographs evaluated. In other words, when PAOT is held constant, if the photograph depicts a PAOT value at the high end of the range it will be evaluated as less acceptable than if the photograph depicts a PAOT value at the low end of the range. 


\section{Table 3-3}

Summary of Photograph Sequence Coefficients for Model 2

\begin{tabular}{lc}
\hline Photograph Sequence & Coefficient \\
\hline${\text { Intercept }\left(\text { Presented } 1^{\text {st }}\right)}^{\text {Presented } 2^{\text {nd }}}$ & 5.63 \\
Presented $3^{\text {rd }}$ & -.41 \\
Presented $4^{\text {th }}$ & -.55 \\
${\text { Presented } 5^{\text {th }}}^{\text {Pr }}$ & -1.14 \\
\hline
\end{tabular}




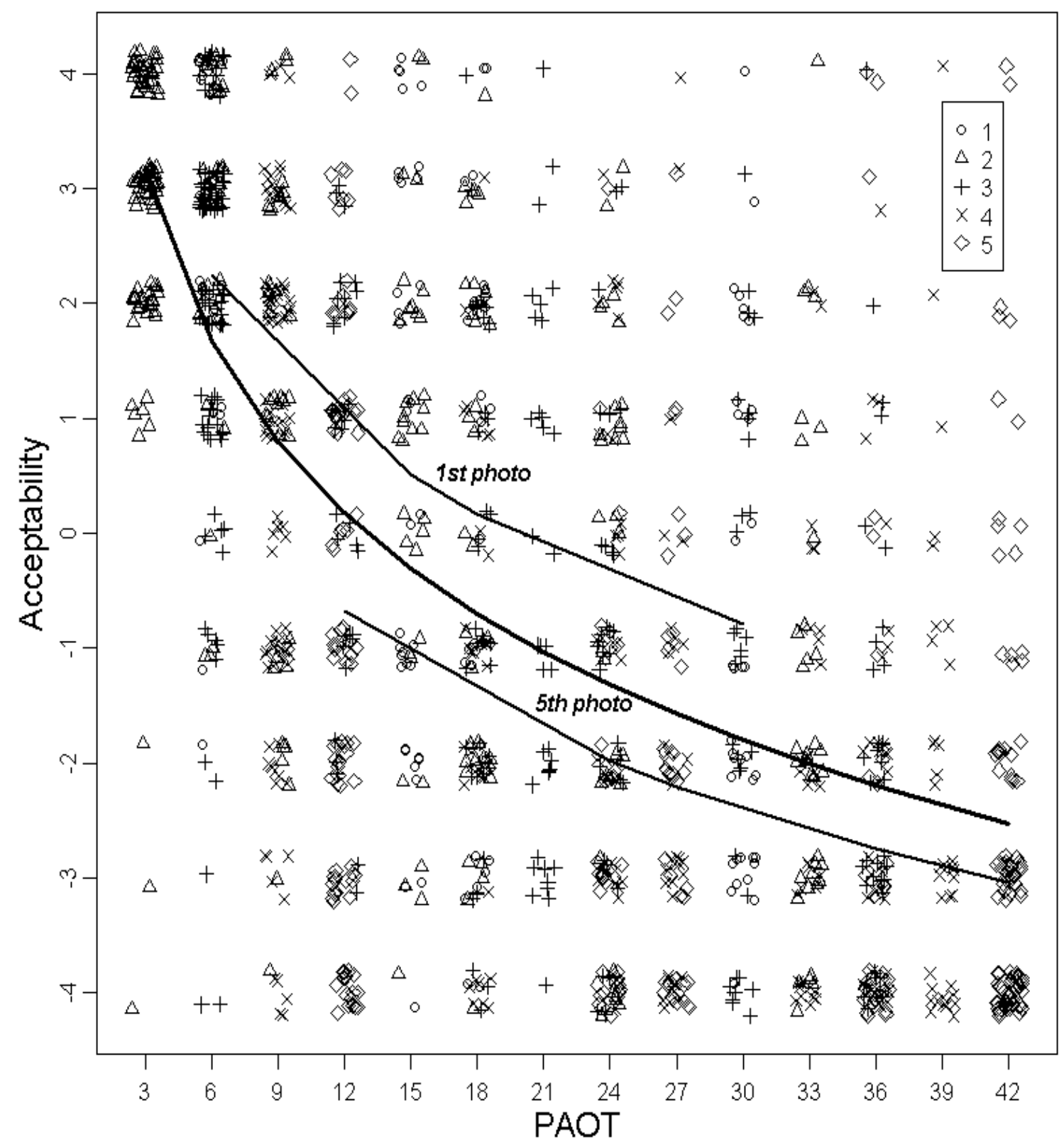

Figure 3-4. Plot of raw data comparing acceptability and PAOT with trend lines for $\log _{10}$ PAOT (middle line), $\log _{10}$ PAOT and $1^{\text {st }}$ photo presented (top line), and $\log _{10}$ PAOT and $5^{\text {th }}$ photo presented (bottom line). 


\section{Discussion}

The purpose of this chapter was to investigate the effect PAOT range had on acceptability ratings of PAOT when using visual based outdoor recreation research methods. A raw data plot (Figure 3-3) and a comparison of mean acceptability ratings (Table 3-3) suggest range may have affected acceptability ratings, but they do little to inform how or exactly how much.

\section{Linear Models}

Results from Model 2 and Model 3 indicated that both range group and photograph sequence number significantly predicted acceptability ratings. However, results indicated that photograph sequence number was a better predictor of acceptability ratings, with a slightly higher $F$ statistic and fewer degrees of freedom than range group.

Photograph sequence number and range group are very closely related variables, and both variables are related to the larger concept of range effect. Range group relates to an overall trend among the 5-photograph set, while photograph sequence number refers to each individual photograph in the sequence. For example, coefficients related to range group refer to a shift in acceptability ratings for the entire range group set. On the other hand, coefficients related to sequence number refer to each individual photograph in the sequence.

Results indicated that where a photograph occurs in the sequence is more important than the overall PAOT range in terms of the ability to predict 
acceptability ratings. In other words, in terms of predicting acceptability ratings, it is more important to know when in the sequence a photograph of 30 PAOT was shown to a respondent than in which range group the respondent belonged. However, just because photograph sequence number was a better predictor than range group does not mean that PAOT range did not affect acceptability ratings.

Photograph sequence number is a representation of range. For example, suppose we are interested in the evaluation of 20 PAOT for a particular area. These results suggest that, all other things being equal, a five-photograph PAOT sequence depicting $0,5,10,15$, and 20 (Sequence $A$ ) will result in a lower acceptability rating for 20 PAOT than a five-photograph sequence depicting 10 , 20, 30, 40, and 50 (Sequence B). In Sequence A, 20 PAOT are evaluated after evaluating 4 other photographs and, so 20 PAOT represents the high end of the range. However, in Sequence B only one photograph was evaluated before the photograph depicting 20 PAOT, so 20 PAOT represents the low end of the range. Sequence B has a larger range, but the subtractive effect of photograph sequence number on acceptability ratings causes the evaluation of 20 PAOT in Sequence A to be lower than Sequence B.

\section{Conclusion}

Similar to the investigation of order effect, an investigation of range effect indicated that PAOT range did significantly affect acceptability ratings of photographs used in visual methods. The manifestation of range effect occurs through photograph presentation sequence. Results showed there was an 
indirect relationship between when in the sequence a photograph was presented (i.e., $1^{\text {st }}, 2^{\text {nd }}, 3^{\text {rd }}, 4^{\text {th }}$, or $5^{\text {th }}$ ) and acceptability ratings. Furthermore, the subtractive effect of sequence on acceptability ratings existed even when controlling for PAOT.

Photograph sequence number only explained $6 \%$ of the variance in acceptability ratings. The amount of variance in acceptability ratings explained by photograph sequence number $(6 \%)$ does not sound like a lot, and it's true, $\log _{10}$ explains far more of the variance in acceptability ratings. Nevertheless, the implication of the coefficients associated with sequence number offer a different perspective. Depending on whether a photograph was presented first or fifth (i.e., represented the low or high PAOT level of the range), acceptability ratings could have differed by nearly two units of acceptability for the same level of PAOT (Table 3-3). Two units of acceptability is more than $1 / 5$ of the 9 -point acceptability scale.

PAOT provided information about the overall trend in acceptability ratings for all 15 photographs. Photograph acceptability ratings provided information about shifts in acceptability ratings depending on when in the five-photograph set the photograph was presented. Like the investigation of photograph presentation order, an investigation of range effect gives us better insight into the variance of photograph acceptability ratings. Not only does acceptability of PAOT vary due to individual preferences associated with PAOT, investigations of order and range effects showed acceptability of PAOT varied due to the order in which 
photographs were presented and when in the sequence a photograph was presented.

The following chapter (Chapter IV) seeks to provide principles that can be used in future visual based research to minimize unwanted variation in acceptability ratings due to the biases of order and range effect. Chapter IV will also outline future research to answer additional questions raised through the investigation of order and range effect. 


\section{CHAPTER IV}

\section{Future Visual Based Outdoor Recreation Research}

\section{Summary of Findings}

Since its inception, the practice of natural resource management has benefited greatly from scientific advancement. Many social science frameworks and methodologies have emerged throughout the history of natural resource management. Those methodologies that proved sound have stood the test of time, while others have fallen into disuse. Still other methodologies continue to be modified and improved upon as scientists continue to test their merits. Thus is the role of science in relation to the management of natural resources.

With the idea of continual scientific improvement in mind, the purpose of this dissertation was to investigate a common method of measuring the social acceptability of use-level in recreation areas. The method in question is known as the visual based method of outdoor recreation research.

Despite the extent to which visual based methods have been researched, two remaining biases of the visual method were introduced and investigated. The first, order effect bias, refers to the potential effect photograph presentation order may have on photograph acceptability ratings. The second, range effect bias, refers to the potential effect the range of resource conditions depicted may have on photograph acceptability ratings. Both potential biases were investigated within the context of acceptability of people at one time (PAOT). 
Investigation of order effect bias indicated that photograph presentation order significantly affected photograph acceptability ratings. Pseudo-random presentation orders yielded a significantly higher amount of non-monotonic values (values that violate the theoretical relationship between PAOT and acceptability as described in Chapter I). Pseudo-random presentation orders also resulted in up to a 1-unit change in acceptability ratings on a 9-point scale.

Investigation of range effect bias indicated that photograph sequence number (i.e., whether a photo was presented $1^{\text {st }}, 2^{\text {nd }}, 3^{\text {rd }}, 4^{\text {th }}$, or $5^{\text {th }}$ ) also significantly affected photograph acceptability ratings. Sequence number was responsible for an almost 2-unit change in acceptability ratings, meaning that the same level of PAOT could be evaluated 2 units of acceptability lower or higher depending on when in the sequence the photograph was evaluated.

\section{Implications}

The investigations of order and range effects suggest that previous visual based outdoor recreation studies may have yielded significantly different results had those studies utilized pseudo-random presentation sequences or presented respondents with a different range of resource conditions. This claim brings into question the function of evaluative standards (i.e., norms) within the context of outdoor recreation, which ultimately leads to questions regarding the existence of recreation norms. However, whether or not evaluative standards used in outdoor recreation research are actually norms should not be confused with whether or not evaluative standards are useful to managers. 
The advocates for recreation norms tend to rely on arguments of utility when challenged on the shortcomings of the recreation norm definition.

Normative data can still be informative even if they do not indicate the existence of a norm. In fact, the norm (i.e., the consensus of evaluations) may actually be of little value to recreation researchers and managers. For example, there may be a great deal of consensus that 100 people is evaluated unfavorably or 0 people is evaluated favorably, but this doesn't tell us a great deal about how much use can be allowed before people begin feeling too crowded.

Recreation researchers use a normative approach (i.e., asking about attitudes and preferences and then aggregating the data) for measuring evaluative standards, but they may not necessarily be interested in what the actual norms are. Consequently, decisions are often made regarding levels of use for which a norm doesn't clearly exist. Instead, recreation researchers determine the range of acceptable conditions and managers make decisions based on those results. In the end, normative research informs decisions, it does not make them.

\section{Suggested Methodological Improvements}

The purpose of investigating photograph order and range effect within visual based methods was to explore how visual based methods could be improved upon by testing for the presence of photograph presentation order and range effects. In exploring the nuances of the relationship between acceptability and PAOT within the context of photograph presentation order and PAOT range, 
several aspects of the visual method emerged that could potentially be improved upon. Results from investigations of order and range effects suggest a number of methodological modifications that merit further investigation. The results from Chapter II \& III suggest the following methodological improvements:

1. Exclude the photograph depicting 0 PAOT.

When designing survey instruments, social scientists continually strive to reduce the burden on respondents in terms of time required to complete a given questionnaire. The consistency of evaluative responses associated with photographs depicting 0 PAOT are such they negate the need for them. Photographs containing 0 PAOT also somewhat conflict with the evaluative question generally associated with the photograph (i.e., "Please rate the photographs in terms of the acceptability of people at one time"). Evaluating the absence of people may be more difficult for respondents to do than evaluating photographs containing people. Photographs with 0 PAOT also may not reflect management objectives. Recreation areas exist, at least partially, as a place for people to recreate. Lastly, 0 PAOT photographs make comparisons with other photographs problematic as was experienced when attempting to take the $\log _{10}$ PAOT. As shown in Chapter II and Chapter III, $\log _{10}$ PAOT was an important predictor of acceptability.

2. Present photographs in random presentation orders.

While it's true that the investigation of order effect bias in Chapter II indicated presentation order only slightly affected acceptability ratings (e.g., 
photograph presentation order explained about $2 \%$ of the variance in acceptability ratings), the potential for a larger effect exists. It may seem logical to avoid random photograph presentation orders because random presentation order can affect acceptability ratings, but that is exactly the reason why random orders should be utilized. Random photograph presentation orders can provide additional information about the existence of an evaluative standard. For example, if presentation order has little or no effect, further support for the standard is provided, and vice versa.

3. Select a broad PAOT range and present photographs depicting subsets of PAOT ranges.

Similar to the suggestion above, range was shown to significantly affect photograph acceptability ratings. Presenting subsets of the larger PAOT range can provide information about the existence of valid evaluative standard.

4. When analyzing acceptability curves, incorporate the use of raw data plots. Raw data plots can be informative concerning the relationship between PAOT and acceptability. In Chapter II, ANOVA analyses indicated no significant differences in mean evaluations between photograph presentation orders within each photograph group. However, a plot of the raw data (Figure 2-2, p. 27) belied the conclusion that order had no effect on acceptability ratings. Additional analyses indicated there was a main effect of photograph presentation order across photograph evaluation trends. 
Visual based studies tend to look at a relatively small section of data (i.e., mean acceptability ratings), but a more comprehensive investigation of results can yield additional insights into the relationship between PAOT and acceptability.

5. Compare photograph evaluation trends rather than mean acceptability ratings for each photograph.

As was shown in Chapter II, ANOVAs comparing mean acceptability ratings may not be robust enough to detect differences in evaluation trends between photograph presentation orders. Linear analyses comparing evaluation trends between the entire set of photographs may be better able to detect the effects of photograph presentation order.

\section{Limitations and Future Research}

The work contained in this dissertation adds to the growing body of recreation research on the measurement and conceptualization of evaluative use standards. However, this dissertation is by no means an exhaustive investigation of potential biases within the visual based method of outdoor recreation research. The limitations of the work described here suggest future avenues of research.

The investigations of order and range effect biases described in Chapters II and III both took place in a laboratory setting. Replication of both of these studies under field conditions may add further credence to their conclusions. On the other hand, first-hand knowledge of the site depicted in the study photographs may drastically alter the results. After all, the inherent nature of 
norms is that they are site-specific (i.e., while norms are shared among individuals, they depend on specific situational, and managerial variables and may not be generalizable to other recreation sites).

Visual based methods can be changed to acoustical based methods by replacing photographs with audio clips (Pilcher, Newman \& Manning, 2009). In these studies, sound clips are played back which contain varying amounts of a particular sound (e.g., aircraft, people, automobiles), or varying levels of a sound (i.e., dBA). It is currently unknown whether order or range may affect evaluations of sound clips. Replicating these studies with the use of sound clips instead of photographs could form the basis of investigations of order and range effects in acoustical based recreation research.

\section{Concluding Remarks}

The results from research contained in this dissertation answer some questions pertaining to the relationship between acceptability and PAOT, but many other questions are also raised. As use of national parks continues to rise, the relevance of use-level related research will continue to increase. While we may never fully understand the nature of recreation experiences, the learning process of science will continue to yield significant contributions toward a more comprehensive understanding. As the statistician George Box once said, "All models are wrong, but some are useful." In other words, while the current models describing the recreation experience may be incomplete, they can continue to provide useful information on use-related recreation resource issues. 


\section{LITERATURE CITED}

Bateson, J., \& Hui, M. (1992). The ecological validity of photographic slides and videotapes in simulating the service setting. Journal of Consumer Research 19, 271-81.

Bosselmann, P. \& Craik, K. H. (1987). Perceptual simulations of environments. Methods in Environmental and Behavioral Research. Van Nostrand: New York, 162-190.

Bradburn, N. M., \& Mason, W. M. (1964). The effect of question order on responses. Journal of Marketing Research, 1(4), 57-61.

Chenoweth, R. (1990). Image capture technology and aesthetic regulation of landscapes adjacent to public lands. Managing America's enduring wilderness resource. St. Paul: University of Minnesota, 563-68.

Fishbein, M., \& Ajzen, I. (1975). Belief, attitude, intention, and behavior: An introduction to theory and research. Reading, MA: Addison-Wesley.

Gibbs, K. (1977). Economics and administrative regulations of outdoor recreation use. Outdoor Recreation: Advances in Application of Economics. USDA Forest Service General Technical Report WO-2, 98-104.

Hall, T. E., \& Roggenbuck, J. W. (2002). Response format effects in questions about norms: Implications for the reliability and validity of the normative approach. Leisure Science, 24, 325-37.

Heberlein, T. (1977). Density, crowding and satisfaction: Sociological studies for determining carrying capacitates. Proceedings: River Recreation Management and Research Symposium. General Technical Report. NC28. St. Paul, MN: USDA Forest Service, 67-76.

Heywood, J. (1996). Conventions, emerging norms, and norms in outdoor recreation. Leisure Sciences 18, 355-63.

Heywood, J. L. (2000) Current approaches to norms research. (pp. 260-264) In Cole, D. N., McCool, S. F., Borrie, W. T., O'Loughlin, J. (Eds.), Wilderness science in a time of change conference - Volume 4: Wilderness visitors, experiences, and visitor management; 1999 May 23-27, Missoula, MT. Proceedings RMS-P-15-VOL-4. Ogden, UT: US Department of Agriculture, Forest Service, Rocky Mountain Research Station. 
Hershberger, R., \& Cass, R. (1974). Predicting user responses to building. Manenvironment interactions: Evaluations and applications. Stroudsbury, PA: Dowden, Hutchinson \& Ross, 117-34.

Homans, G. C. (1950). The human group. New York: Hardcourt Brace.

Jackson, J. (1965). Structural characteristics of norms. Current studies in social psychology. New York, NY: Holt, Rinehart and Winston, Inc., 301-9.

Jacobi, C., \& Manning, R. (1999). Crowding and conflict on the carriage roads of Acadia National Park: An application of the Visitor Experience and Resource Protection framework, Park Science, 19, 22-26.

Mascia, M. B., Brosius, J. P., Dobson, T., A., Forbes, B. C., Horowitz, L., McKean, M. A., \& Turner, N. J. (2003). Conservation and the social sciences. Conservation Biology, 17(3), 649-650.

Manning, R. E. (1985). Crowding norms in backcountry settings: A review and synthesis. Journal of Leisure Research, 17(2), 75-89.

Manning, R. E. (1999). Studies in Outdoor Recreation: Search and Research for Satisfaction. Corvallis, OR: Oregon State University Press. Manning, R.E., \& Lawson, S. (2002). Carrying capacity as "informed judgment": The values of science and the science of values. Environmental Management, 30(2), 157-168.

Manning, R. E. (2007). Parks and carrying capacity: Commons without tragedy. Washington, DC: Island Press.

Manning, R. E., \& Freidmund, W.A. (2004). Use of visual methods to measure standards of quality for parks and outdoor recreation. Journal of Leisure Research, 36(4), 557-579.

Manning, R., Freimund, W., \& Marion, J. (2004). Research to Support Application of the Visitor Experience and Resource Protection (VERP) Framework to Backcountry Planning at Zion National Park. Burlington: University of Vermont, Park Studies Lab.

Manning, R. E., \& Lawson, S. (2002). Carrying capacity as "informed judgment": The values of science and the science of values. Environmental Management, 30(2), 157-168. 
Manning, R., Lawson, S., \& Frymier, L. (1999). Navigating the confluence of two streams of social research: Contingent valuation and normative standards. Research in Human Ecology, 6(1), 35-48.

Manning, R., Lawson, S., Newman, P., Laven, D., \& Valliere, W. (2002). Methodological issues in measuring crowding-related norms in outdoor recreation. Leisure Sciences, 24, 339-48.

Manning, R., Leung, Y., \& Budrul, M. (2005). Research to support management of visitor carrying capacity at Boston Harbor Islands. Northeastern Naturalist, 12, 201-20.

Manning, R. E., Lime, D. W., Friemund, W. A., \& Pitt, D. G. (1996). Crowding norms at frontcountry sites: A visual approach to setting standards of quality. Leisure Sciences, 18, 39-59.

Manning, R., Lime, D., Hof, M., \& Freimund, W. (1995). The carrying capacity of national parks: Theory and application. Proceedings of the Conference on Innovations and Challenges in the Management of Visitor Opportunities in Parks and Protected Areas. Waterloo, Canada: University of Waterloo, 921.

Manning, R., Valliere, W., Wang, B., Lawson, S., \& Newman, P. (2003). Estimating day use social carrying capacity in Yosemite National Park. Leisure: The Journal of the Canadian Association for Leisure Studies, 27, 77-102.

Manning, R., Valliere, B., Minteer, B., Wang, B., \& Jacobi, C. (2000). Crowding in parks and outdoor recreation: A theoretical, empirical, and managerial analysis. Journal of Parks and Recreation Administration, 18, 57-72.

Manning, R., Valliere, W., Wang, B., \& Jacobi, C. (1999). Crowding norms: Alternative measurement approaches. Leisure Sciences, 21, 219-229.

Manning, R., Valliere, W., Wang, B., Lawson, S., \& Newman, P. (2003). Estimating day use social carrying capacity in Yosemite National Park. Leisure: The Journal of the Canadian Association for Leisure Studies, 27, 77-102.

Manning, R., Wang, B., Valliere, W., Lawson, S., \& Newman, P. (2002). Research to estimate and manage carrying capacity of a tourist attraction: A study of Alcatraz Island. Journal of Sustainable Tourism, 10, 388-464.

McDonald, C. D. (1996). Normative perspective on outdoor recreation behavior: Introductory comments. Leisure Sciences, 18(1), 1-6. 
McFarland, S. G. (1981). Effects of question order on survey responses. The Public Opinion Quarterly, 45(2), 208-215.

McKechnie, G. E. (1977). Simulation techniques in environmental psychology. Perspectives on Environmental and Behavior: Theory, Research and Applications, ed. Daniel Stokols. New York: Plenum, 169-189.

Moore, d. W. (2002). Measuring new types of question-order effects: Additive and subtractive. The Public Opinion Quarterly, 66(1), 80-91.

Mosteller, F., \& Tukey, J. (1977). Data analysis and regression. Reading, MA: Addison-Wesley.

Parsons, T. (1951). The social system. Glencoe: Free Press.

Pilcher, E. J., Newman, P., \& Manning, R. E. (2009). Understanding and Managing Experiential Aspects of Sound at Muir Woods National Monument. Environmental Management, 28(3), 424-35.

Schwarz, N., Hippler, H. J., Deutsch, B., \& Strack, F. (1985). Response scales: Effects of category range on reported behavior and comparative judgments. Public Opinion Quarterly, 49, 388-395.

Shelby, B. (1980). Crowding models for backcountry recreation. Land Economics, 56, 43-55.

Shelby, B., \& Harris, R. (1985). Comparing methods for determining visitor evaluations of ecological impacts: Site visits, photographs, and written descriptions. Journal of Leisure Research, 17, 57-67.

Shelby, B., \& Heberlein, T. A. (1986). Carrying capacity in recreation settings. Corvallis, OR: Oregon State University Press.

Shelby, B., \& Shindler, B. (1992). Interest group standards for ecological impacts at wilderness campsites. Leisure Sciences, 14, 17-27.

Shelby, B., \& Vaske, J. (1991). Using normative data to develop evaluative standards for resource management: A comment on three recent papers. Journal of Leisure Research 23, 173-87.

Shelby, B., Vaske, J., \& Donnelly, M. (1996). Norms, standards and natural resources. Leisure Sciences, 18, 103-23. 
Thayer, M. A. (1981). Contingent valuation techniques for assessing environmental impacts: Further evidence. Journal of Environmental Economics and Management, 8, 27-44.

Valliere, W., \& Manning, R. (2003). Applying the Visitor Experience and Resource Protection (VERP) framework to cultural resources in national parks. Proceedings of the 2002 Northeastern Recreation Research Symposium. USDA Forest Service General Technical Report NE-302, 234-38.

Vaske, J., Donnelly, M., \& Shelby, B. (1993). Establishing management standards: Selected examples of the normative approach. Environmental Management, 17, 629-43.

Vaske, J., Graefe, A., Shelby, B., \& Heberlein, T. (1986). Backcountry encounter norms: Theory, method, and empirical evidence. Journal of Leisure Research 18, 137-53.

Zaller, J., \& Feldman, S. (1992). A simple theory of the survey response: Answering questions versus revealing preferences. American Journal of Political Science, 36(3), 579-616. 


\section{APPENDIX A: Order Effect Data}

ID = Identification number of respondent

Order $=$ Photograph presentation order $($ Table 2-1, p. 24)

Acceptability $=$ Acceptability rating $(-4$ to +4$)$

Photo \# = Photograph evaluated (Figure 2-1, p. 23)

\begin{tabular}{|c|c|c|c|}
\hline ID & Order & Acceptability & Photograph \# \\
\hline 1 & 1 & 3 & 1 \\
\hline 1 & 1 & 2 & 2 \\
\hline 1 & 1 & 0 & 3 \\
\hline 1 & 1 & -2 & 4 \\
\hline 1 & 1 & -4 & 5 \\
\hline 2 & 1 & 4 & 1 \\
\hline 2 & 1 & 2 & 2 \\
\hline 2 & 1 & 0 & 3 \\
\hline 2 & 1 & -2 & 4 \\
\hline 2 & 1 & -4 & 5 \\
\hline 3 & 1 & 4 & 1 \\
\hline 3 & 1 & 4 & 2 \\
\hline 3 & 1 & 3 & 3 \\
\hline 3 & 1 & 3 & 4 \\
\hline 3 & 1 & 2 & 5 \\
\hline 4 & 1 & 4 & 1 \\
\hline 4 & 1 & -1 & 2 \\
\hline 4 & 1 & -4 & 3 \\
\hline 4 & 1 & -4 & 4 \\
\hline 4 & 1 & -4 & 5 \\
\hline 5 & 1 & 2 & 1 \\
\hline 5 & 1 & 2 & 2 \\
\hline 5 & 1 & 2 & 3 \\
\hline 5 & 1 & 1 & 4 \\
\hline 5 & 1 & -1 & 5 \\
\hline 6 & 1 & 4 & 1 \\
\hline 6 & 1 & 3 & 2 \\
\hline 6 & 1 & 2 & 3 \\
\hline 6 & 1 & -1 & 4 \\
\hline
\end{tabular}




\begin{tabular}{|c|c|c|c|}
\hline 6 & 1 & -4 & 5 \\
\hline 7 & 1 & 4 & 1 \\
\hline 7 & 1 & -2 & 2 \\
\hline 7 & 1 & -3 & 3 \\
\hline 7 & 1 & -4 & 4 \\
\hline 7 & 1 & -4 & 5 \\
\hline 8 & 1 & 4 & 1 \\
\hline 8 & 1 & 3 & 2 \\
\hline 8 & 1 & 1 & 3 \\
\hline 8 & 1 & -2 & 4 \\
\hline 8 & 1 & -4 & 5 \\
\hline 9 & 1 & 4 & 1 \\
\hline 9 & 1 & 1 & 2 \\
\hline 9 & 1 & -2 & 3 \\
\hline 9 & 1 & -4 & 4 \\
\hline 9 & 1 & -4 & 5 \\
\hline 10 & 1 & 4 & 1 \\
\hline 10 & 1 & 2 & 2 \\
\hline 10 & 1 & 0 & 3 \\
\hline 10 & 1 & -1 & 4 \\
\hline 10 & 1 & -3 & 5 \\
\hline 11 & 1 & 4 & 1 \\
\hline 11 & 1 & 2 & 2 \\
\hline 11 & 1 & 1 & 3 \\
\hline 11 & 1 & -1 & 4 \\
\hline 11 & 1 & -3 & 5 \\
\hline 12 & 1 & 4 & 1 \\
\hline 12 & 1 & 3 & 2 \\
\hline 12 & 1 & -1 & 3 \\
\hline 12 & 1 & -1 & 4 \\
\hline 12 & 1 & -2 & 5 \\
\hline 13 & 1 & 2 & 1 \\
\hline 13 & 1 & 1 & 2 \\
\hline 13 & 1 & 0 & 3 \\
\hline 13 & 1 & 0 & 4 \\
\hline 13 & 1 & -1 & 5 \\
\hline 14 & 1 & 4 & 1 \\
\hline 14 & 1 & 0 & 2 \\
\hline 14 & 1 & 0 & 3 \\
\hline 14 & 1 & -3 & 4 \\
\hline 14 & 1 & -4 & 5 \\
\hline 15 & 1 & 4 & 1 \\
\hline 15 & 1 & 3 & 2 \\
\hline 15 & 1 & -1 & 3 \\
\hline 15 & 1 & -3 & 4 \\
\hline 15 & 1 & -4 & 5 \\
\hline 16 & 1 & 3 & 1 \\
\hline
\end{tabular}




\begin{tabular}{|c|c|c|c|}
\hline 16 & 1 & 0 & 2 \\
\hline 16 & 1 & -2 & 3 \\
\hline 16 & 1 & -3 & 4 \\
\hline 16 & 1 & -4 & 5 \\
\hline 17 & 1 & 3 & 1 \\
\hline 17 & 1 & 3 & 2 \\
\hline 17 & 1 & 1 & 3 \\
\hline 17 & 1 & 0 & 4 \\
\hline 17 & 1 & -1 & 5 \\
\hline 18 & 1 & 4 & 1 \\
\hline 18 & 1 & 2 & 2 \\
\hline 18 & 1 & 0 & 3 \\
\hline 18 & 1 & -2 & 4 \\
\hline 18 & 1 & -4 & 5 \\
\hline 19 & 1 & 3 & 1 \\
\hline 19 & 1 & 2 & 2 \\
\hline 19 & 1 & 2 & 3 \\
\hline 19 & 1 & 1 & 4 \\
\hline 19 & 1 & 0 & 5 \\
\hline 20 & 1 & 4 & 1 \\
\hline 20 & 1 & 4 & 2 \\
\hline 20 & 1 & 4 & 3 \\
\hline 20 & 1 & 4 & 4 \\
\hline 20 & 1 & 4 & 5 \\
\hline 21 & 1 & -3 & 1 \\
\hline 21 & 1 & 1 & 2 \\
\hline 21 & 1 & -1 & 3 \\
\hline 21 & 1 & -2 & 4 \\
\hline 21 & 1 & -4 & 5 \\
\hline 22 & 1 & 4 & 1 \\
\hline 22 & 1 & 4 & 2 \\
\hline 22 & 1 & 4 & 3 \\
\hline 22 & 1 & 2 & 4 \\
\hline 22 & 1 & -2 & 5 \\
\hline 23 & 1 & 4 & 1 \\
\hline 23 & 1 & -1 & 2 \\
\hline 23 & 1 & -4 & 3 \\
\hline 23 & 1 & -4 & 4 \\
\hline 23 & 1 & -4 & 5 \\
\hline 24 & 1 & 4 & 1 \\
\hline 24 & 1 & 3 & 2 \\
\hline 24 & 1 & 2 & 3 \\
\hline 24 & 1 & -1 & 4 \\
\hline 24 & 1 & -3 & 5 \\
\hline 25 & 1 & 4 & 1 \\
\hline 25 & 1 & 1 & 2 \\
\hline 25 & 1 & -2 & 3 \\
\hline
\end{tabular}




\begin{tabular}{|c|c|c|c|}
\hline 25 & 1 & -3 & 4 \\
\hline 25 & 1 & -4 & 5 \\
\hline 26 & 1 & 4 & 1 \\
\hline 26 & 1 & 1 & 2 \\
\hline 26 & 1 & -1 & 3 \\
\hline 26 & 1 & -3 & 4 \\
\hline 26 & 1 & -4 & 5 \\
\hline 27 & 1 & 2 & 1 \\
\hline 27 & 1 & 1 & 2 \\
\hline 27 & 1 & 0 & 3 \\
\hline 27 & 1 & -3 & 4 \\
\hline 27 & 1 & -4 & 5 \\
\hline 28 & 1 & 4 & 1 \\
\hline 28 & 1 & 4 & 2 \\
\hline 28 & 1 & 3 & 3 \\
\hline 28 & 1 & 2 & 4 \\
\hline 28 & 1 & 1 & 5 \\
\hline 29 & 1 & 2 & 1 \\
\hline 29 & 1 & 1 & 2 \\
\hline 29 & 1 & 1 & 3 \\
\hline 29 & 1 & -2 & 4 \\
\hline 29 & 1 & -3 & 5 \\
\hline 30 & 1 & 2 & 1 \\
\hline 30 & 1 & 1 & 2 \\
\hline 30 & 1 & 1 & 3 \\
\hline 30 & 1 & 0 & 4 \\
\hline 30 & 1 & -1 & 5 \\
\hline 31 & 1 & 3 & 1 \\
\hline 31 & 1 & 3 & 2 \\
\hline 31 & 1 & 2 & 3 \\
\hline 31 & 1 & 0 & 4 \\
\hline 31 & 1 & -1 & 5 \\
\hline 32 & 1 & 4 & 1 \\
\hline 32 & 1 & 2 & 2 \\
\hline 32 & 1 & -2 & 3 \\
\hline 32 & 1 & -4 & 4 \\
\hline 32 & 1 & -4 & 5 \\
\hline 33 & 1 & 3 & 1 \\
\hline 33 & 1 & -1 & 2 \\
\hline 33 & 1 & -3 & 3 \\
\hline 33 & 1 & -3 & 4 \\
\hline 33 & 1 & -4 & 5 \\
\hline 34 & 2 & 3 & 1 \\
\hline 34 & 2 & 1 & 2 \\
\hline 34 & 2 & 3 & 3 \\
\hline 34 & 2 & 2 & 4 \\
\hline 34 & 2 & 0 & 5 \\
\hline
\end{tabular}




\begin{tabular}{|c|c|c|c|}
\hline 35 & 2 & 4 & 1 \\
\hline 35 & 2 & 1 & 2 \\
\hline 35 & 2 & 1 & 3 \\
\hline 35 & 2 & -3 & 4 \\
\hline 35 & 2 & -4 & 5 \\
\hline 36 & 2 & 2 & 1 \\
\hline 36 & 2 & -3 & 2 \\
\hline 36 & 2 & 3 & 3 \\
\hline 36 & 2 & -4 & 4 \\
\hline 36 & 2 & -4 & 5 \\
\hline 37 & 2 & -1 & 1 \\
\hline 37 & 2 & 2 & 2 \\
\hline 37 & 2 & 1 & 3 \\
\hline 37 & 2 & 3 & 4 \\
\hline 37 & 2 & 1 & 5 \\
\hline 38 & 2 & & 1 \\
\hline 38 & 2 & 2 & 2 \\
\hline 38 & 2 & -4 & 3 \\
\hline 38 & 2 & -3 & 4 \\
\hline 38 & 2 & 4 & 5 \\
\hline 39 & 2 & 4 & 1 \\
\hline 39 & 2 & -1 & 2 \\
\hline 39 & 2 & -4 & 3 \\
\hline 39 & 2 & -4 & 4 \\
\hline 39 & 2 & -4 & 5 \\
\hline 40 & 2 & 4 & 1 \\
\hline 40 & 2 & 4 & 2 \\
\hline 40 & 2 & -1 & 3 \\
\hline 40 & 2 & 1 & 4 \\
\hline 40 & 2 & -4 & 5 \\
\hline 41 & 2 & 4 & 1 \\
\hline 41 & 2 & -2 & 2 \\
\hline 41 & 2 & -3 & 3 \\
\hline 41 & 2 & -4 & 4 \\
\hline 41 & 2 & -4 & 5 \\
\hline 42 & 2 & 4 & 1 \\
\hline 42 & 2 & 4 & 2 \\
\hline 42 & 2 & 3 & 3 \\
\hline 42 & 2 & 2 & 4 \\
\hline 42 & 2 & -1 & 5 \\
\hline 43 & 2 & 4 & 1 \\
\hline 43 & 2 & 3 & 2 \\
\hline 43 & 2 & -1 & 3 \\
\hline 43 & 2 & -2 & 4 \\
\hline 43 & 2 & -3 & 5 \\
\hline 44 & 2 & 4 & 1 \\
\hline 44 & 2 & -3 & 2 \\
\hline
\end{tabular}




\begin{tabular}{|c|c|c|c|}
\hline 44 & 2 & -1 & 3 \\
\hline 44 & 2 & -4 & 4 \\
\hline 44 & 2 & -4 & 5 \\
\hline 45 & 2 & 4 & 1 \\
\hline 45 & 2 & 1 & 2 \\
\hline 45 & 2 & -1 & 3 \\
\hline 45 & 2 & -2 & 4 \\
\hline 45 & 2 & -3 & 5 \\
\hline 46 & 2 & 4 & 1 \\
\hline 46 & 2 & 0 & 2 \\
\hline 46 & 2 & 3 & 3 \\
\hline 46 & 2 & -3 & 4 \\
\hline 46 & 2 & -4 & 5 \\
\hline 47 & 2 & 4 & 1 \\
\hline 47 & 2 & -1 & 2 \\
\hline 47 & 2 & -2 & 3 \\
\hline 47 & 2 & -4 & 4 \\
\hline 47 & 2 & -4 & 5 \\
\hline 48 & 2 & 4 & 1 \\
\hline 48 & 2 & -3 & 2 \\
\hline 48 & 2 & -2 & 3 \\
\hline 48 & 2 & -3 & 4 \\
\hline 48 & 2 & -4 & 5 \\
\hline 49 & 2 & 4 & 1 \\
\hline 49 & 2 & 3 & 2 \\
\hline 49 & 2 & 0 & 3 \\
\hline 49 & 2 & 1 & 4 \\
\hline 49 & 2 & -1 & 5 \\
\hline 50 & 2 & 3 & 1 \\
\hline 50 & 2 & 1 & 2 \\
\hline 50 & 2 & -1 & 3 \\
\hline 50 & 2 & -2 & 4 \\
\hline 50 & 2 & -3 & 5 \\
\hline 51 & 2 & 4 & 1 \\
\hline 51 & 2 & 3 & 2 \\
\hline 51 & 2 & -4 & 3 \\
\hline 51 & 2 & -4 & 4 \\
\hline 51 & 2 & -4 & 5 \\
\hline 52 & 2 & & 1 \\
\hline 52 & 2 & 4 & 2 \\
\hline 52 & 2 & & 3 \\
\hline 52 & 2 & 4 & 4 \\
\hline 52 & 2 & 3 & 5 \\
\hline 53 & 2 & 3 & 1 \\
\hline 53 & 2 & 4 & 2 \\
\hline 53 & 2 & 4 & 3 \\
\hline 53 & 2 & 2 & 4 \\
\hline
\end{tabular}




\begin{tabular}{|c|c|c|c|}
\hline 53 & 2 & 1 & 5 \\
\hline 54 & 2 & 2 & 1 \\
\hline 54 & 2 & -1 & 2 \\
\hline 54 & 2 & -1 & 3 \\
\hline 54 & 2 & -3 & 4 \\
\hline 54 & 2 & -4 & 5 \\
\hline 55 & 2 & 4 & 1 \\
\hline 55 & 2 & -1 & 2 \\
\hline 55 & 2 & -2 & 3 \\
\hline 55 & 2 & -3 & 4 \\
\hline 55 & 2 & -4 & 5 \\
\hline 56 & 2 & 3 & 1 \\
\hline 56 & 2 & -3 & 2 \\
\hline 56 & 2 & -3 & 3 \\
\hline 56 & 2 & -4 & 4 \\
\hline 56 & 2 & -4 & 5 \\
\hline 57 & 2 & 4 & 1 \\
\hline 57 & 2 & 1 & 2 \\
\hline 57 & 2 & 0 & 3 \\
\hline 57 & 2 & -1 & 4 \\
\hline 57 & 2 & -1 & 5 \\
\hline 58 & 2 & 4 & 1 \\
\hline 58 & 2 & -1 & 2 \\
\hline 58 & 2 & -2 & 3 \\
\hline 58 & 2 & -4 & 4 \\
\hline 58 & 2 & -4 & 5 \\
\hline 59 & 2 & 4 & 1 \\
\hline 59 & 2 & 3 & 2 \\
\hline 59 & 2 & 0 & 3 \\
\hline 59 & 2 & -3 & 4 \\
\hline 59 & 2 & -4 & 5 \\
\hline 60 & 2 & 3 & 1 \\
\hline 60 & 2 & 4 & 2 \\
\hline 60 & 2 & 4 & 3 \\
\hline 60 & 2 & 2 & 4 \\
\hline 60 & 2 & -1 & 5 \\
\hline 61 & 2 & 4 & 1 \\
\hline 61 & 2 & 3 & 2 \\
\hline 61 & 2 & -2 & 3 \\
\hline 61 & 2 & -4 & 4 \\
\hline 61 & 2 & -4 & 5 \\
\hline 62 & 2 & 4 & 1 \\
\hline 62 & 2 & 1 & 2 \\
\hline 62 & 2 & -2 & 3 \\
\hline 62 & 2 & -3 & 4 \\
\hline 62 & 2 & -4 & 5 \\
\hline 63 & 2 & 4 & 1 \\
\hline
\end{tabular}




\begin{tabular}{|c|c|c|c|}
\hline 63 & 2 & 2 & 2 \\
\hline 63 & 2 & 2 & 3 \\
\hline 63 & 2 & 1 & 4 \\
\hline 63 & 2 & 1 & 5 \\
\hline 64 & 2 & 4 & 1 \\
\hline 64 & 2 & 2 & 2 \\
\hline 64 & 2 & 0 & 3 \\
\hline 64 & 2 & -2 & 4 \\
\hline 64 & 2 & -4 & 5 \\
\hline 65 & 3 & & 1 \\
\hline 65 & 3 & 3 & 2 \\
\hline 65 & 3 & 2 & 3 \\
\hline 65 & 3 & 3 & 4 \\
\hline 65 & 3 & -1 & 5 \\
\hline 66 & 3 & 4 & 1 \\
\hline 66 & 3 & -3 & 2 \\
\hline 66 & 3 & -4 & 3 \\
\hline 66 & 3 & -4 & 4 \\
\hline 66 & 3 & -4 & 5 \\
\hline 67 & 3 & 4 & 1 \\
\hline 67 & 3 & 2 & 2 \\
\hline 67 & 3 & -3 & 3 \\
\hline 67 & 3 & -3 & 4 \\
\hline 67 & 3 & -4 & 5 \\
\hline 68 & 3 & 4 & 1 \\
\hline 68 & 3 & 3 & 2 \\
\hline 68 & 3 & 2 & 3 \\
\hline 68 & 3 & 1 & 4 \\
\hline 68 & 3 & 1 & 5 \\
\hline 69 & 3 & 2 & 1 \\
\hline 69 & 3 & 3 & 2 \\
\hline 69 & 3 & 2 & 3 \\
\hline 69 & 3 & 2 & 4 \\
\hline 69 & 3 & 1 & 5 \\
\hline 70 & 3 & 4 & 1 \\
\hline 70 & 3 & 3 & 2 \\
\hline 70 & 3 & -2 & 3 \\
\hline 70 & 3 & -3 & 4 \\
\hline 70 & 3 & -4 & 5 \\
\hline 71 & 3 & 4 & 1 \\
\hline 71 & 3 & 3 & 2 \\
\hline 71 & 3 & 2 & 3 \\
\hline 71 & 3 & -2 & 4 \\
\hline 71 & 3 & -3 & 5 \\
\hline 72 & 3 & 4 & 1 \\
\hline 72 & 3 & 4 & 2 \\
\hline 72 & 3 & 2 & 3 \\
\hline
\end{tabular}




\begin{tabular}{|c|c|c|c|}
\hline 72 & 3 & 2 & 4 \\
\hline 72 & 3 & -3 & 5 \\
\hline 73 & 3 & 1 & 1 \\
\hline 73 & 3 & -1 & 2 \\
\hline 73 & 3 & -4 & 3 \\
\hline 73 & 3 & -4 & 4 \\
\hline 73 & 3 & -4 & 5 \\
\hline 74 & 3 & 3 & 1 \\
\hline 74 & 3 & 4 & 2 \\
\hline 74 & 3 & 4 & 3 \\
\hline 74 & 3 & 3 & 4 \\
\hline 74 & 3 & 1 & 5 \\
\hline 75 & 3 & 4 & 1 \\
\hline 75 & 3 & 3 & 2 \\
\hline 75 & 3 & -2 & 3 \\
\hline 75 & 3 & -3 & 4 \\
\hline 75 & 3 & -4 & 5 \\
\hline 76 & 3 & 4 & 1 \\
\hline 76 & 3 & 2 & 2 \\
\hline 76 & 3 & 0 & 3 \\
\hline 76 & 3 & -2 & 4 \\
\hline 76 & 3 & -3 & 5 \\
\hline 77 & 3 & 4 & 1 \\
\hline 77 & 3 & 1 & 2 \\
\hline 77 & 3 & 0 & 3 \\
\hline 77 & 3 & 0 & 4 \\
\hline 77 & 3 & -2 & 5 \\
\hline 78 & 3 & 4 & 1 \\
\hline 78 & 3 & 4 & 2 \\
\hline 78 & 3 & 1 & 3 \\
\hline 78 & 3 & & 4 \\
\hline 78 & 3 & 2 & 5 \\
\hline 79 & 3 & 4 & 1 \\
\hline 79 & 3 & 4 & 2 \\
\hline 79 & 3 & 0 & 3 \\
\hline 79 & 3 & -3 & 4 \\
\hline 79 & 3 & -3 & 5 \\
\hline 80 & 3 & -2 & 1 \\
\hline 80 & 3 & -2 & 2 \\
\hline 80 & 3 & 3 & 3 \\
\hline 80 & 3 & 3 & 4 \\
\hline 80 & 3 & & 5 \\
\hline 81 & 3 & 3 & 1 \\
\hline 81 & 3 & 2 & 2 \\
\hline 81 & 3 & -1 & 3 \\
\hline 81 & 3 & -2 & 4 \\
\hline 81 & 3 & -3 & 5 \\
\hline
\end{tabular}




\begin{tabular}{|c|c|c|c|}
\hline 82 & 3 & 4 & 1 \\
\hline 82 & 3 & -2 & 2 \\
\hline 82 & 3 & -3 & 3 \\
\hline 82 & 3 & -3 & 4 \\
\hline 82 & 3 & -4 & 5 \\
\hline 83 & 3 & -2 & 1 \\
\hline 83 & 3 & -3 & 2 \\
\hline 83 & 3 & 2 & 3 \\
\hline 83 & 3 & -1 & 4 \\
\hline 83 & 3 & 0 & 5 \\
\hline 84 & 3 & 4 & 1 \\
\hline 84 & 3 & 1 & 2 \\
\hline 84 & 3 & 1 & 3 \\
\hline 84 & 3 & -1 & 4 \\
\hline 84 & 3 & -1 & 5 \\
\hline 85 & 3 & 4 & 1 \\
\hline 85 & 3 & 2 & 2 \\
\hline 85 & 3 & -3 & 3 \\
\hline 85 & 3 & -3 & 4 \\
\hline 85 & 3 & -4 & 5 \\
\hline 86 & 3 & 4 & 1 \\
\hline 86 & 3 & 3 & 2 \\
\hline 86 & 3 & -3 & 3 \\
\hline 86 & 3 & -3 & 4 \\
\hline 86 & 3 & -4 & 5 \\
\hline 87 & 3 & 2 & 1 \\
\hline 87 & 3 & 4 & 2 \\
\hline 87 & 3 & 2 & 3 \\
\hline 87 & 3 & 1 & 4 \\
\hline 87 & 3 & -2 & 5 \\
\hline 88 & 3 & 4 & 1 \\
\hline 88 & 3 & 1 & 2 \\
\hline 88 & 3 & -3 & 3 \\
\hline 88 & 3 & -4 & 4 \\
\hline 88 & 3 & -4 & 5 \\
\hline 89 & 3 & 4 & 1 \\
\hline 89 & 3 & -1 & 2 \\
\hline 89 & 3 & -1 & 3 \\
\hline 89 & 3 & -2 & 4 \\
\hline 89 & 3 & -3 & 5 \\
\hline 90 & 3 & 4 & 1 \\
\hline 90 & 3 & 3 & 2 \\
\hline 90 & 3 & -2 & 3 \\
\hline 90 & 3 & -2 & 4 \\
\hline 90 & 3 & -4 & 5 \\
\hline 91 & 3 & 4 & 1 \\
\hline 91 & 3 & 2 & 2 \\
\hline
\end{tabular}




\begin{tabular}{|c|c|c|c|}
\hline 91 & 3 & -3 & 3 \\
\hline 91 & 3 & -3 & 4 \\
\hline 91 & 3 & -4 & 5 \\
\hline 92 & 3 & 3 & 1 \\
\hline 92 & 3 & 2 & 2 \\
\hline 92 & 3 & 2 & 3 \\
\hline 92 & 3 & 0 & 4 \\
\hline 92 & 3 & 1 & 5 \\
\hline 93 & 3 & 4 & 1 \\
\hline 93 & 3 & 1 & 2 \\
\hline 93 & 3 & -3 & 3 \\
\hline 93 & 3 & -4 & 4 \\
\hline 93 & 3 & -4 & 5 \\
\hline 94 & 3 & 4 & 1 \\
\hline 94 & 3 & 3 & 2 \\
\hline 94 & 3 & -2 & 3 \\
\hline 94 & 3 & -3 & 4 \\
\hline 94 & 3 & -4 & 5 \\
\hline 95 & 3 & 3 & 1 \\
\hline 95 & 3 & 3 & 2 \\
\hline 95 & 3 & 0 & 3 \\
\hline 95 & 3 & 0 & 4 \\
\hline 95 & 3 & -1 & 5 \\
\hline 96 & 3 & 4 & 1 \\
\hline 96 & 3 & 4 & 2 \\
\hline 96 & 3 & 3 & 3 \\
\hline 96 & 3 & 2 & 4 \\
\hline 96 & 3 & -2 & 5 \\
\hline 97 & 4 & 3 & 1 \\
\hline 97 & 4 & -1 & 2 \\
\hline 97 & 4 & -3 & 3 \\
\hline 97 & 4 & -3 & 4 \\
\hline 97 & 4 & -4 & 5 \\
\hline 98 & 4 & 3 & 1 \\
\hline 98 & 4 & 2 & 2 \\
\hline 98 & 4 & 0 & 3 \\
\hline 98 & 4 & -1 & 4 \\
\hline 98 & 4 & -4 & 5 \\
\hline 99 & 4 & 4 & 1 \\
\hline 99 & 4 & 3 & 2 \\
\hline 99 & 4 & 2 & 3 \\
\hline 99 & 4 & 1 & 4 \\
\hline 99 & 4 & -2 & 5 \\
\hline 100 & 4 & 4 & 1 \\
\hline 100 & 4 & 2 & 2 \\
\hline 100 & 4 & -1 & 3 \\
\hline 100 & 4 & -3 & 4 \\
\hline
\end{tabular}




\begin{tabular}{|c|c|c|c|}
\hline 100 & 4 & -4 & 5 \\
\hline 101 & 4 & 4 & 1 \\
\hline 101 & 4 & -1 & 2 \\
\hline 101 & 4 & -2 & 3 \\
\hline 101 & 4 & -3 & 4 \\
\hline 101 & 4 & -4 & 5 \\
\hline 102 & 4 & 4 & 1 \\
\hline 102 & 4 & 3 & 2 \\
\hline 102 & 4 & 1 & 3 \\
\hline 102 & 4 & -1 & 4 \\
\hline 102 & 4 & -3 & 5 \\
\hline 103 & 4 & 4 & 1 \\
\hline 103 & 4 & 2 & 2 \\
\hline 103 & 4 & -1 & 3 \\
\hline 103 & 4 & 0 & 4 \\
\hline 103 & 4 & -3 & 5 \\
\hline 104 & 4 & 4 & 1 \\
\hline 104 & 4 & 1 & 2 \\
\hline 104 & 4 & -2 & 3 \\
\hline 104 & 4 & -3 & 4 \\
\hline 104 & 4 & -4 & 5 \\
\hline 105 & 4 & 4 & 1 \\
\hline 105 & 4 & 2 & 2 \\
\hline 105 & 4 & 1 & 3 \\
\hline 105 & 4 & -1 & 4 \\
\hline 105 & 4 & -3 & 5 \\
\hline 106 & 4 & 3 & 1 \\
\hline 106 & 4 & -1 & 2 \\
\hline 106 & 4 & -3 & 3 \\
\hline 106 & 4 & -4 & 4 \\
\hline 106 & 4 & -4 & 5 \\
\hline 107 & 4 & 4 & 1 \\
\hline 107 & 4 & -1 & 2 \\
\hline 107 & 4 & -3 & 3 \\
\hline 107 & 4 & -4 & 4 \\
\hline 107 & 4 & -4 & 5 \\
\hline 108 & 4 & 2 & 1 \\
\hline 108 & 4 & 2 & 2 \\
\hline 108 & 4 & 1 & 3 \\
\hline 108 & 4 & -1 & 4 \\
\hline 108 & 4 & -3 & 5 \\
\hline 109 & 4 & 4 & 1 \\
\hline 109 & 4 & 2 & 2 \\
\hline 109 & 4 & 0 & 3 \\
\hline 109 & 4 & -2 & 4 \\
\hline 109 & 4 & -4 & 5 \\
\hline 110 & 4 & 3 & 1 \\
\hline
\end{tabular}




\begin{tabular}{|c|c|c|c|}
\hline 110 & 4 & 4 & 2 \\
\hline 110 & 4 & 3 & 3 \\
\hline 110 & 4 & 1 & 4 \\
\hline 110 & 4 & -4 & 5 \\
\hline 111 & 4 & 4 & 1 \\
\hline 111 & 4 & 3 & 2 \\
\hline 111 & 4 & 2 & 3 \\
\hline 111 & 4 & 2 & 4 \\
\hline 111 & 4 & 1 & 5 \\
\hline 112 & 4 & 3 & 1 \\
\hline 112 & 4 & 2 & 2 \\
\hline 112 & 4 & -1 & 3 \\
\hline 112 & 4 & -3 & 4 \\
\hline 112 & 4 & -4 & 5 \\
\hline 113 & 4 & 4 & 1 \\
\hline 113 & 4 & -2 & 2 \\
\hline 113 & 4 & -3 & 3 \\
\hline 113 & 4 & -4 & 4 \\
\hline 113 & 4 & -4 & 5 \\
\hline 114 & 4 & 3 & 1 \\
\hline 114 & 4 & 1 & 2 \\
\hline 114 & 4 & 1 & 3 \\
\hline 114 & 4 & 1 & 4 \\
\hline 114 & 4 & -3 & 5 \\
\hline 115 & 4 & 4 & 1 \\
\hline 115 & 4 & 4 & 2 \\
\hline 115 & 4 & 4 & 3 \\
\hline 115 & 4 & 4 & 4 \\
\hline 115 & 4 & -3 & 5 \\
\hline 116 & 4 & 3 & 1 \\
\hline 116 & 4 & 2 & 2 \\
\hline 116 & 4 & -1 & 3 \\
\hline 116 & 4 & -2 & 4 \\
\hline 116 & 4 & -3 & 5 \\
\hline 117 & 4 & 3 & 1 \\
\hline 117 & 4 & -1 & 2 \\
\hline 117 & 4 & 4 & 3 \\
\hline 117 & 4 & 3 & 4 \\
\hline 117 & 4 & 4 & 5 \\
\hline 118 & 4 & 4 & 1 \\
\hline 118 & 4 & 0 & 2 \\
\hline 118 & 4 & -2 & 3 \\
\hline 118 & 4 & -3 & 4 \\
\hline 118 & 4 & -3 & 5 \\
\hline 119 & 4 & -1 & 1 \\
\hline 119 & 4 & -1 & 2 \\
\hline 119 & 4 & 3 & 3 \\
\hline
\end{tabular}




\begin{tabular}{|c|c|c|c|}
\hline 119 & 4 & 1 & 4 \\
\hline 119 & 4 & -1 & 5 \\
\hline 120 & 4 & 4 & 1 \\
\hline 120 & 4 & -1 & 2 \\
\hline 120 & 4 & -2 & 3 \\
\hline 120 & 4 & -4 & 4 \\
\hline 120 & 4 & -4 & 5 \\
\hline 121 & 4 & 4 & 1 \\
\hline 121 & 4 & 1 & 2 \\
\hline 121 & 4 & -1 & 3 \\
\hline 121 & 4 & -3 & 4 \\
\hline 121 & 4 & -4 & 5 \\
\hline 122 & 4 & 4 & 1 \\
\hline 122 & 4 & -3 & 2 \\
\hline 122 & 4 & -4 & 3 \\
\hline 122 & 4 & -4 & 4 \\
\hline 122 & 4 & -4 & 5 \\
\hline 123 & 4 & 4 & 1 \\
\hline 123 & 4 & 1 & 2 \\
\hline 123 & 4 & 2 & 3 \\
\hline 123 & 4 & 2 & 4 \\
\hline 123 & 4 & -1 & 5 \\
\hline 124 & 4 & 3 & 1 \\
\hline 124 & 4 & 2 & 2 \\
\hline 124 & 4 & -2 & 3 \\
\hline 124 & 4 & -2 & 4 \\
\hline 124 & 4 & -3 & 5 \\
\hline 125 & 4 & 4 & 1 \\
\hline 125 & 4 & -1 & 2 \\
\hline 125 & 4 & -3 & 3 \\
\hline 125 & 4 & -4 & 4 \\
\hline 125 & 4 & -4 & 5 \\
\hline 126 & 4 & 3 & 1 \\
\hline 126 & 4 & 3 & 2 \\
\hline 126 & 4 & 0 & 3 \\
\hline 126 & 4 & -2 & 4 \\
\hline 126 & 4 & -3 & 5 \\
\hline 127 & 4 & 4 & 1 \\
\hline 127 & 4 & 1 & 2 \\
\hline 127 & 4 & 0 & 3 \\
\hline 127 & 4 & -1 & 4 \\
\hline 127 & 4 & -4 & 5 \\
\hline 128 & 5 & 4 & 1 \\
\hline 128 & 5 & 4 & 2 \\
\hline 128 & 5 & 2 & 3 \\
\hline 128 & 5 & 1 & 4 \\
\hline 128 & 5 & -3 & 5 \\
\hline
\end{tabular}




\begin{tabular}{|c|c|c|c|}
\hline 129 & 5 & 4 & 1 \\
\hline 129 & 5 & -1 & 2 \\
\hline 129 & 5 & -2 & 3 \\
\hline 129 & 5 & -3 & 4 \\
\hline 129 & 5 & -3 & 5 \\
\hline 130 & 5 & 3 & 1 \\
\hline 130 & 5 & -1 & 2 \\
\hline 130 & 5 & 1 & 3 \\
\hline 130 & 5 & -3 & 4 \\
\hline 130 & 5 & -4 & 5 \\
\hline 131 & 5 & 4 & 1 \\
\hline 131 & 5 & 4 & 2 \\
\hline 131 & 5 & 4 & 3 \\
\hline 131 & 5 & 0 & 4 \\
\hline 131 & 5 & -1 & 5 \\
\hline 132 & 5 & 4 & 1 \\
\hline 132 & 5 & 2 & 2 \\
\hline 132 & 5 & 1 & 3 \\
\hline 132 & 5 & -2 & 4 \\
\hline 132 & 5 & -3 & 5 \\
\hline 133 & 5 & 4 & 1 \\
\hline 133 & 5 & 3 & 2 \\
\hline 133 & 5 & 3 & 3 \\
\hline 133 & 5 & 2 & 4 \\
\hline 133 & 5 & 2 & 5 \\
\hline 134 & 5 & 4 & 1 \\
\hline 134 & 5 & 2 & 2 \\
\hline 134 & 5 & -1 & 3 \\
\hline 134 & 5 & -2 & 4 \\
\hline 134 & 5 & -4 & 5 \\
\hline 135 & 5 & -1 & 1 \\
\hline 135 & 5 & 2 & 2 \\
\hline 135 & 5 & 3 & 3 \\
\hline 135 & 5 & -2 & 4 \\
\hline 135 & 5 & -3 & 5 \\
\hline 136 & 5 & 4 & 1 \\
\hline 136 & 5 & 4 & 2 \\
\hline 136 & 5 & 4 & 3 \\
\hline 136 & 5 & 4 & 4 \\
\hline 136 & 5 & 4 & 5 \\
\hline 137 & 5 & 4 & 1 \\
\hline 137 & 5 & 1 & 2 \\
\hline 137 & 5 & -2 & 3 \\
\hline 137 & 5 & -3 & 4 \\
\hline 137 & 5 & -4 & 5 \\
\hline 138 & 5 & 4 & 1 \\
\hline 138 & 5 & -2 & 2 \\
\hline
\end{tabular}




\begin{tabular}{|c|c|c|c|}
\hline 138 & 5 & -3 & 3 \\
\hline 138 & 5 & -4 & 4 \\
\hline 138 & 5 & -4 & 5 \\
\hline 139 & 5 & 4 & 1 \\
\hline 139 & 5 & 4 & 2 \\
\hline 139 & 5 & 1 & 3 \\
\hline 139 & 5 & 0 & 4 \\
\hline 139 & 5 & -1 & 5 \\
\hline 140 & 5 & 4 & 1 \\
\hline 140 & 5 & 2 & 2 \\
\hline 140 & 5 & 2 & 3 \\
\hline 140 & 5 & -1 & 4 \\
\hline 140 & 5 & -3 & 5 \\
\hline 141 & 5 & 4 & 1 \\
\hline 141 & 5 & -2 & 2 \\
\hline 141 & 5 & -3 & 3 \\
\hline 141 & 5 & -4 & 4 \\
\hline 141 & 5 & -4 & 5 \\
\hline 142 & 5 & 4 & 1 \\
\hline 142 & 5 & 2 & 2 \\
\hline 142 & 5 & -2 & 3 \\
\hline 142 & 5 & -3 & 4 \\
\hline 142 & 5 & -3 & 5 \\
\hline 143 & 5 & 4 & 1 \\
\hline 143 & 5 & 2 & 2 \\
\hline 143 & 5 & 0 & 3 \\
\hline 143 & 5 & -3 & 4 \\
\hline 143 & 5 & -4 & 5 \\
\hline 144 & 5 & 4 & 1 \\
\hline 144 & 5 & 0 & 2 \\
\hline 144 & 5 & -2 & 3 \\
\hline 144 & 5 & -3 & 4 \\
\hline 144 & 5 & -4 & 5 \\
\hline 145 & 5 & 4 & 1 \\
\hline 145 & 5 & -1 & 2 \\
\hline 145 & 5 & -3 & 3 \\
\hline 145 & 5 & -4 & 4 \\
\hline 145 & 5 & -4 & 5 \\
\hline 146 & 5 & 4 & 1 \\
\hline 146 & 5 & 2 & 2 \\
\hline 146 & 5 & -2 & 3 \\
\hline 146 & 5 & -3 & 4 \\
\hline 146 & 5 & -4 & 5 \\
\hline 147 & 5 & 4 & 1 \\
\hline 147 & 5 & -1 & 2 \\
\hline 147 & 5 & -2 & 3 \\
\hline 147 & 5 & -3 & 4 \\
\hline
\end{tabular}




\begin{tabular}{|c|c|c|c|}
\hline 147 & 5 & -4 & 5 \\
\hline 148 & 5 & 3 & 1 \\
\hline 148 & 5 & 1 & 2 \\
\hline 148 & 5 & -1 & 3 \\
\hline 148 & 5 & -1 & 4 \\
\hline 148 & 5 & & 5 \\
\hline 149 & 5 & 2 & 1 \\
\hline 149 & 5 & 4 & 2 \\
\hline 149 & 5 & 2 & 3 \\
\hline 149 & 5 & 3 & 4 \\
\hline 149 & 5 & 4 & 5 \\
\hline 150 & 5 & 3 & 1 \\
\hline 150 & 5 & 3 & 2 \\
\hline 150 & 5 & -1 & 3 \\
\hline 150 & 5 & 0 & 4 \\
\hline 150 & 5 & 0 & 5 \\
\hline 151 & 5 & 4 & 1 \\
\hline 151 & 5 & -1 & 2 \\
\hline 151 & 5 & -3 & 3 \\
\hline 151 & 5 & -4 & 4 \\
\hline 151 & 5 & -4 & 5 \\
\hline 152 & 5 & 4 & 1 \\
\hline 152 & 5 & 3 & 2 \\
\hline 152 & 5 & 1 & 3 \\
\hline 152 & 5 & -3 & 4 \\
\hline 152 & 5 & -4 & 5 \\
\hline 153 & 5 & -1 & 1 \\
\hline 153 & 5 & -3 & 2 \\
\hline 153 & 5 & -3 & 3 \\
\hline 153 & 5 & -4 & 4 \\
\hline 153 & 5 & -4 & 5 \\
\hline 154 & 5 & 4 & 1 \\
\hline 154 & 5 & 2 & 2 \\
\hline 154 & 5 & -2 & 3 \\
\hline 154 & 5 & -3 & 4 \\
\hline 154 & 5 & -3 & 5 \\
\hline 155 & 5 & 3 & 1 \\
\hline 155 & 5 & 1 & 2 \\
\hline 155 & 5 & -1 & 3 \\
\hline 155 & 5 & -2 & 4 \\
\hline 155 & 5 & -2 & 5 \\
\hline 156 & 5 & 2 & 1 \\
\hline 156 & 5 & 3 & 2 \\
\hline 156 & 5 & 1 & 3 \\
\hline 156 & 5 & 1 & 4 \\
\hline 156 & 5 & -1 & 5 \\
\hline 157 & 6 & 3 & 1 \\
\hline
\end{tabular}




\begin{tabular}{|c|c|c|c|}
\hline 157 & 6 & 0 & 2 \\
\hline 157 & 6 & -1 & 3 \\
\hline 157 & 6 & -1 & 4 \\
\hline 157 & 6 & -2 & 5 \\
\hline 158 & 6 & 4 & 1 \\
\hline 158 & 6 & 3 & 2 \\
\hline 158 & 6 & 2 & 3 \\
\hline 158 & 6 & 2 & 4 \\
\hline 158 & 6 & 1 & 5 \\
\hline 159 & 6 & 4 & 1 \\
\hline 159 & 6 & 3 & 2 \\
\hline 159 & 6 & 1 & 3 \\
\hline 159 & 6 & -2 & 4 \\
\hline 159 & 6 & -2 & 5 \\
\hline 160 & 6 & 4 & 1 \\
\hline 160 & 6 & -1 & 2 \\
\hline 160 & 6 & -2 & 3 \\
\hline 160 & 6 & -3 & 4 \\
\hline 160 & 6 & -4 & 5 \\
\hline 161 & 6 & 4 & 1 \\
\hline 161 & 6 & 2 & 2 \\
\hline 161 & 6 & 0 & 3 \\
\hline 161 & 6 & -1 & 4 \\
\hline 161 & 6 & -2 & 5 \\
\hline 162 & 6 & 2 & 1 \\
\hline 162 & 6 & 2 & 2 \\
\hline 162 & 6 & 1 & 3 \\
\hline 162 & 6 & -1 & 4 \\
\hline 162 & 6 & -2 & 5 \\
\hline 163 & 6 & 3 & 1 \\
\hline 163 & 6 & 1 & 2 \\
\hline 163 & 6 & 0 & 3 \\
\hline 163 & 6 & 0 & 4 \\
\hline 163 & 6 & -2 & 5 \\
\hline 164 & 6 & 0 & 1 \\
\hline 164 & 6 & -1 & 2 \\
\hline 164 & 6 & -1 & 3 \\
\hline 164 & 6 & -2 & 4 \\
\hline 164 & 6 & -2 & 5 \\
\hline 165 & 6 & 4 & 1 \\
\hline 165 & 6 & 2 & 2 \\
\hline 165 & 6 & 1 & 3 \\
\hline 165 & 6 & -1 & 4 \\
\hline 165 & 6 & -1 & 5 \\
\hline 166 & 6 & 4 & 1 \\
\hline 166 & 6 & 2 & 2 \\
\hline 166 & 6 & 1 & 3 \\
\hline
\end{tabular}




\begin{tabular}{|c|c|c|c|}
\hline 166 & 6 & -1 & 4 \\
\hline 166 & 6 & -2 & 5 \\
\hline 167 & 6 & 4 & 1 \\
\hline 167 & 6 & 1 & 2 \\
\hline 167 & 6 & -1 & 3 \\
\hline 167 & 6 & -2 & 4 \\
\hline 167 & 6 & -2 & 5 \\
\hline 168 & 6 & 4 & 1 \\
\hline 168 & 6 & 4 & 2 \\
\hline 168 & 6 & 4 & 3 \\
\hline 168 & 6 & 3 & 4 \\
\hline 168 & 6 & 3 & 5 \\
\hline 169 & 6 & 4 & 1 \\
\hline 169 & 6 & 3 & 2 \\
\hline 169 & 6 & 1 & 3 \\
\hline 169 & 6 & -2 & 4 \\
\hline 169 & 6 & -3 & 5 \\
\hline 170 & 6 & 4 & 1 \\
\hline 170 & 6 & 3 & 2 \\
\hline 170 & 6 & 2 & 3 \\
\hline 170 & 6 & 1 & 4 \\
\hline 170 & 6 & -1 & 5 \\
\hline 171 & 6 & 4 & 1 \\
\hline 171 & 6 & 1 & 2 \\
\hline 171 & 6 & -3 & 3 \\
\hline 171 & 6 & -4 & 4 \\
\hline 171 & 6 & -4 & 5 \\
\hline 172 & 6 & 4 & 1 \\
\hline 172 & 6 & 3 & 2 \\
\hline 172 & 6 & 3 & 3 \\
\hline 172 & 6 & 2 & 4 \\
\hline 172 & 6 & 2 & 5 \\
\hline 173 & 6 & 4 & 1 \\
\hline 173 & 6 & 3 & 2 \\
\hline 173 & 6 & 2 & 3 \\
\hline 173 & 6 & 0 & 4 \\
\hline 173 & 6 & -1 & 5 \\
\hline 174 & 6 & 2 & 1 \\
\hline 174 & 6 & -1 & 2 \\
\hline 174 & 6 & -1 & 3 \\
\hline 174 & 6 & -3 & 4 \\
\hline 174 & 6 & -3 & 5 \\
\hline 175 & 6 & 4 & 1 \\
\hline 175 & 6 & 2 & 2 \\
\hline 175 & 6 & -1 & 3 \\
\hline 175 & 6 & -2 & 4 \\
\hline 175 & 6 & -3 & 5 \\
\hline
\end{tabular}




\begin{tabular}{|c|c|c|c|}
\hline 176 & 6 & 3 & 1 \\
\hline 176 & 6 & 2 & 2 \\
\hline 176 & 6 & 0 & 3 \\
\hline 176 & 6 & -1 & 4 \\
\hline 176 & 6 & -2 & 5 \\
\hline 177 & 6 & 4 & 1 \\
\hline 177 & 6 & 2 & 2 \\
\hline 177 & 6 & -2 & 3 \\
\hline 177 & 6 & -3 & 4 \\
\hline 177 & 6 & -4 & 5 \\
\hline 178 & 6 & 4 & 1 \\
\hline 178 & 6 & 2 & 2 \\
\hline 178 & 6 & 1 & 3 \\
\hline 178 & 6 & -1 & 4 \\
\hline 178 & 6 & -2 & 5 \\
\hline 179 & 6 & 4 & 1 \\
\hline 179 & 6 & 4 & 2 \\
\hline 179 & 6 & 0 & 3 \\
\hline 179 & 6 & -2 & 4 \\
\hline 179 & 6 & -3 & 5 \\
\hline 180 & 6 & 4 & 1 \\
\hline 180 & 6 & 4 & 2 \\
\hline 180 & 6 & 0 & 3 \\
\hline 180 & 6 & -2 & 4 \\
\hline 180 & 6 & -4 & 5 \\
\hline 181 & 6 & 3 & 1 \\
\hline 181 & 6 & 3 & 2 \\
\hline 181 & 6 & 2 & 3 \\
\hline 181 & 6 & 0 & 4 \\
\hline 181 & 6 & -2 & 5 \\
\hline 182 & 6 & 4 & 1 \\
\hline 182 & 6 & 2 & 2 \\
\hline 182 & 6 & 1 & 3 \\
\hline 182 & 6 & 0 & 4 \\
\hline 182 & 6 & -1 & 5 \\
\hline 183 & 6 & 4 & 1 \\
\hline 183 & 6 & 4 & 2 \\
\hline 183 & 6 & 2 & 3 \\
\hline 183 & 6 & -1 & 4 \\
\hline 183 & 6 & -2 & 5 \\
\hline 184 & 6 & 4 & 1 \\
\hline 184 & 6 & 3 & 2 \\
\hline 184 & 6 & 2 & 3 \\
\hline 184 & 6 & -1 & 4 \\
\hline 184 & 6 & -2 & 5 \\
\hline 185 & 6 & 4 & 1 \\
\hline 185 & 6 & 3 & 2 \\
\hline
\end{tabular}




\begin{tabular}{|c|c|c|c|}
\hline 185 & 6 & 1 & 3 \\
\hline 185 & 6 & 0 & 4 \\
\hline 185 & 6 & -2 & 5 \\
\hline 186 & 6 & 4 & 1 \\
\hline 186 & 6 & 3 & 2 \\
\hline 186 & 6 & 1 & 3 \\
\hline 186 & 6 & -1 & 4 \\
\hline 186 & 6 & -1 & 5 \\
\hline 187 & 6 & 3 & 1 \\
\hline 187 & 6 & 1 & 2 \\
\hline 187 & 6 & 0 & 3 \\
\hline 187 & 6 & 0 & 4 \\
\hline 187 & 6 & -2 & 5 \\
\hline
\end{tabular}




\section{APPENDIX B: Order Effect R Script}

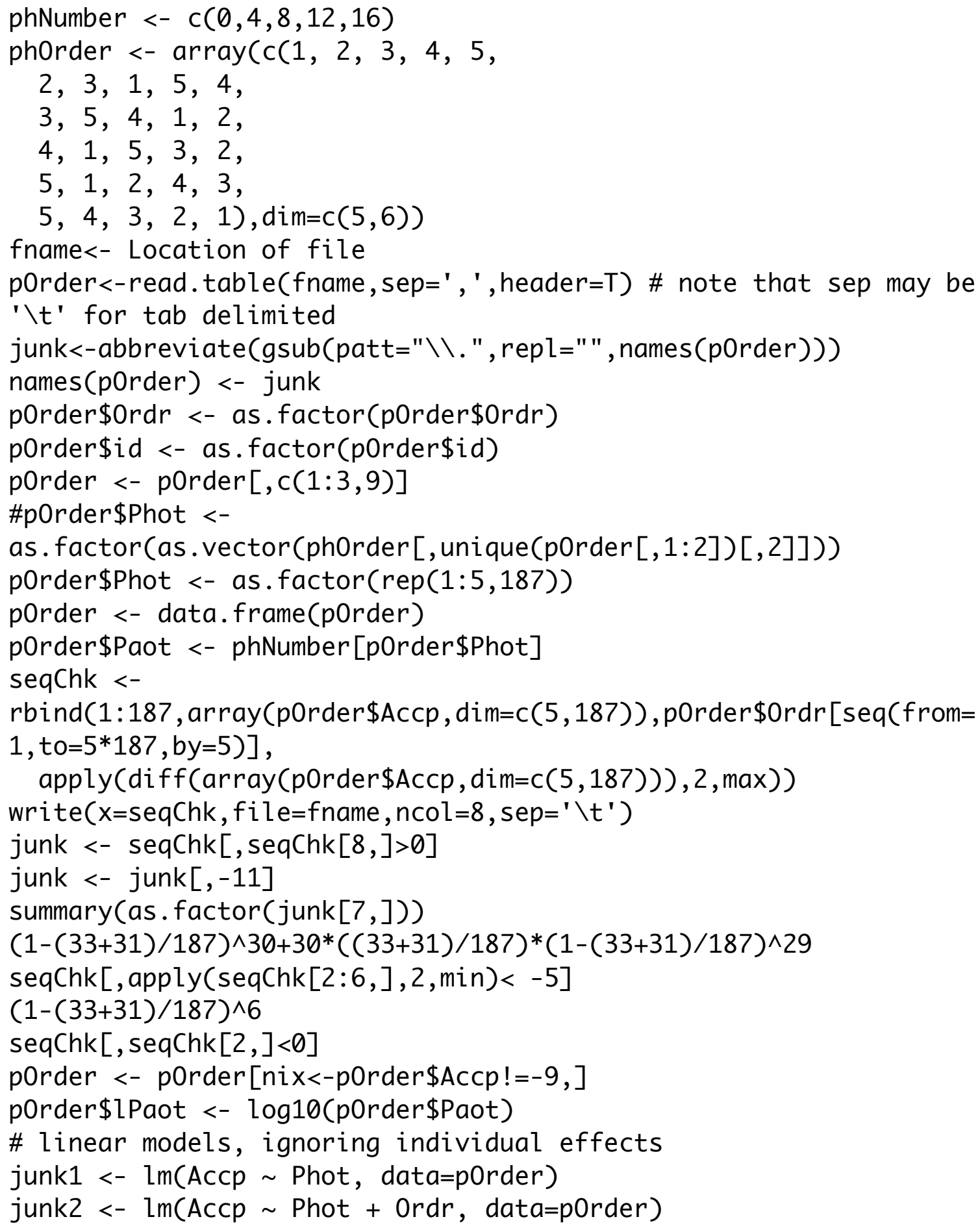




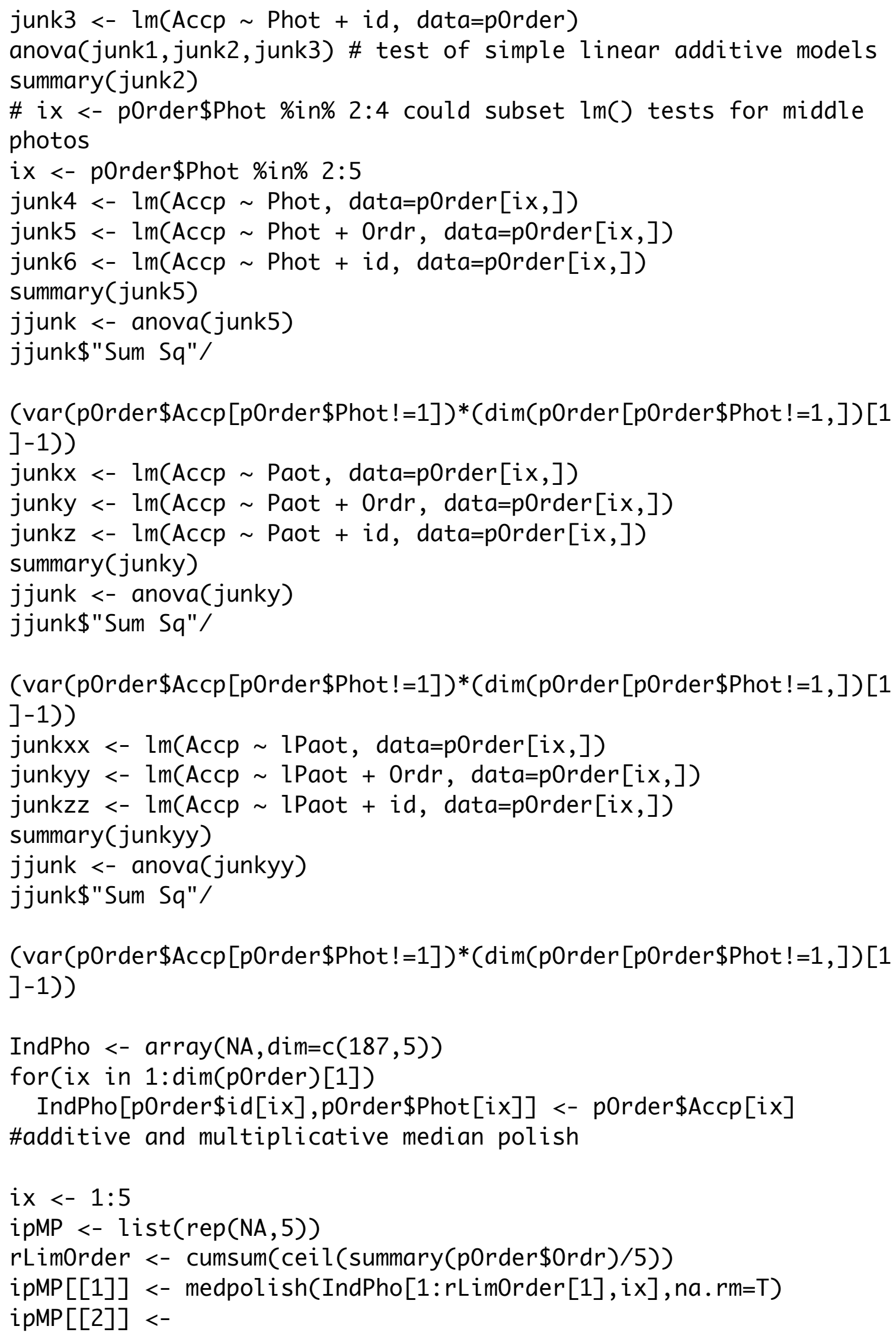


medpolish(IndPho[(rLim0rder[1]+1): rLim0rder[2], ix], na. rm=T) ipMP $[[3]]<-$ medpolish(IndPho[(rLim0rder [2]+1):rLim0rder [3], ix], na.rm=T) ipMP $[[4]]<-$ medpolish(IndPho[(rLim0rder[3]+1):rLim0rder [4], ix], na.rm=T) ipMP $[[5]]<-$ medpolish(IndPho[(rLim0rder [4]+1):rLim0rder [5], ix], na.rm=T) ipMP $[[6]]<-$ medpol ish(IndPho[(rLim0rder [5]+1):rLim0rder [6], ix] , na.rm=T) sapply (ipMP, "[", "col") sapply (ipMP, " [", "row") nCurves <- $\operatorname{array}($ unlist(sapply(ipMP, "[", "col")), dim=c(5,6))\#+ nCurves2 <array (sapply (split (p0rder $\$ A c c p$, paste (p0rder $\$ 0 r d r$, p0rder $\$$ Phot, sep= "")), mean), $\operatorname{dim}=c(5,6))$

\# sapply(sapply(sapply(ipMP, "[", "row"), mean), rep , 5)

plot (apply (nCurves, 1, mean), yl im=c (-

4.5,4.5), xlab="Photo",ylab="Acceptability", $x \lim =c(0.5,6), x a x s={ }^{\prime} i$ ' , type $=$ 'n') polygon( $\mathrm{x}=\operatorname{par}() \$ \operatorname{usr}[\mathrm{c}(1: 2,2: 1,1)], \mathrm{y}=\operatorname{par}() \$ \operatorname{usr}[\mathrm{c}(3,3,4,4,3)], \mathrm{col}="$ gray $70 ")$ points(apply(nCurves, 1 , mean), pch=' +' , cex=2)

for(iOrd in 1:dim(nCurves)[2]) \{ points (i0rd/12+(1:5), nCurves [, i0rd], col=clrs[i0rd]) lines( (1:5), nCurves2 [,i0rd], col=clrs[i0rd]) \}

legend $(x=1, y=-2, \operatorname{leg}=1: 6, \operatorname{col}=\operatorname{clrs}[1: 6], p c h=1)$

ord0ffset <- sapply(sapply(ipMP, "[", "row"), mean)

indOffset <- unlist(sapply(ipMP, "[", "row"))

p0rder\$Iofs <- ind0ffset[p0rder\$id]\#-ord0ffset[p0rder\$0rdr]

junk7 <- $\operatorname{lm}($ Accp $\sim$ Phot, data=p0rder $)$

junk8 <- Im(Accp Phot + Iofs, data=p0rder)

junk9 <- Im(Accp Phot + Iofs + Ordr, data=p0rder)

anova(junk 8$)$

summary (junk8)

accAdj <- junk[names(junk)=="Iofs"] * ind0ffset

accAdj <- accAdj +

$c(0$, junk [substring (names (junk) $, 1,4)==" 0 r d r "])$ [pOrder $\$ 0 r d r[$ ! dupl ic ated(p0rder\$id)]]

yRng <- -6:6

windows (hei=8.5, wid=11)

$\operatorname{par}(\mathrm{mfrow}=\mathrm{c}(1,2), \operatorname{mar}=c(3.5,4.5,0,0), \mathrm{oma}=c(0,0,2,2), \mathrm{mgp}=c(2,0.7,0)$ 


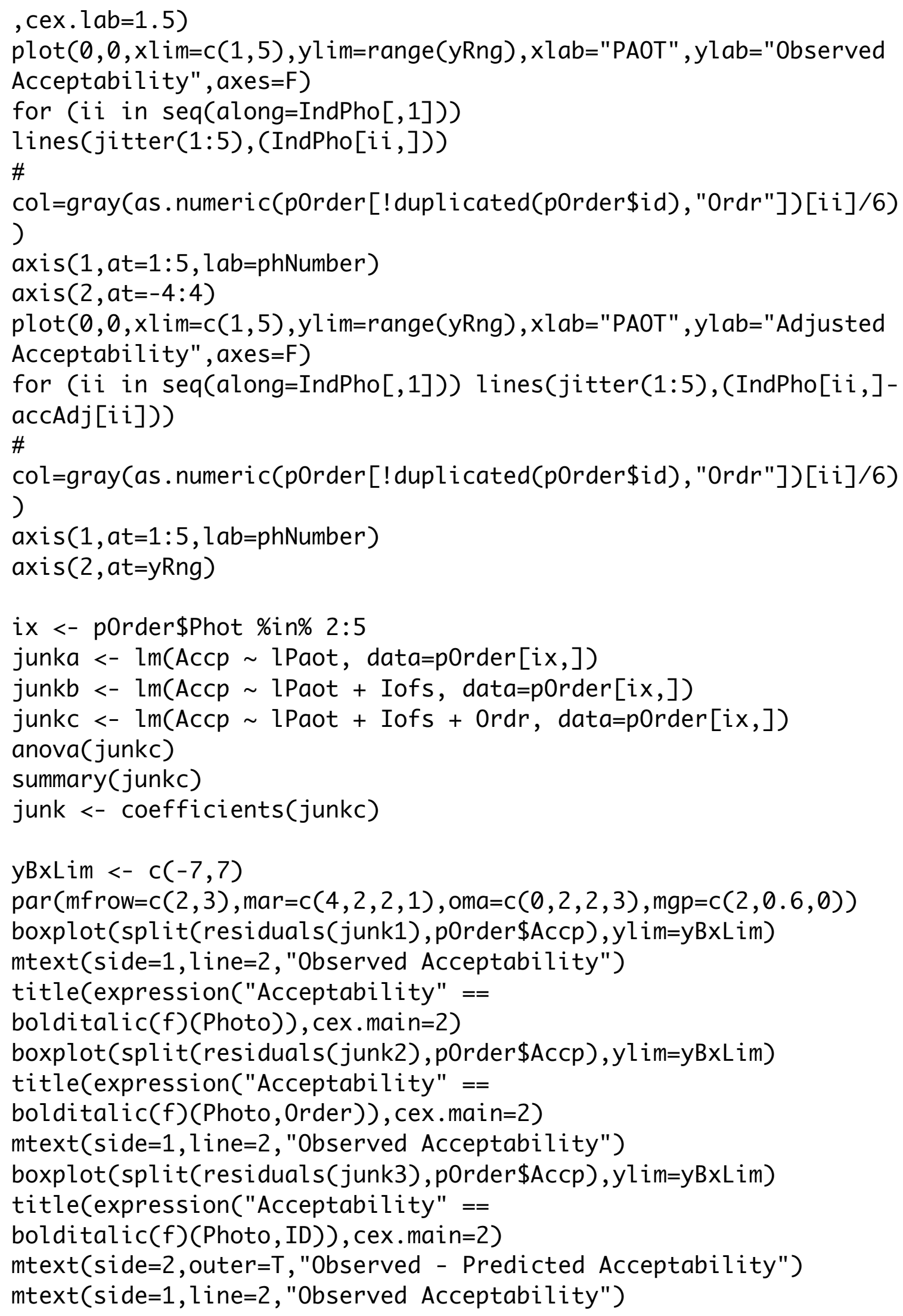


windows (wid $=2 * 5.5$, hei $=2 * 5.5 * 8.5 / 11)$

$\operatorname{par}(m f r o w=c(1,2), \operatorname{mar}=c(4,2,2,1)$, oma $=c(0,2,4,3), m g p=c(2,0.6,0)$, cex .axis=1.5)

boxplot(split(residuals(junk9), p0rder $\$ A c c p$ ), yl im=yBxLim)

mtext ( side=1, line=2, "Observed Acceptability", cex=1.5)

title("All Photos", cex.main=1.5)

boxplot(split(residuals(junkc),p0rder $\$ A c c p[i x]$ ), yl im=yBxLim)

title("Photos 2 - 5", cex.main=1.5)

title(expression("Acceptability" == bolditalic(f)(Photo, "Median

Polish Offset",Order)),

outer $=T$, cex . main $=2$ )

mtext ( side $=2$, outer $=\mathrm{T}$, "Observed - Predicted

Acceptability", cex=1.5)

mtext ( side $=1$, line=2, "Observed Acceptability" , cex=1.5)

dev.off() \# closes the window

junkd <- gam(Accp s(Paot, Ordr, $d f=8)+$ Iofs, data=pOrder[ix, ]) plot (p0rder\$Phot [ix], junkd\$smooth, pch=".")

\# ix <- which(p0rder\$Phot==ph0rder[1,p0rder\$0rdr]) \# focused on the first photo seen

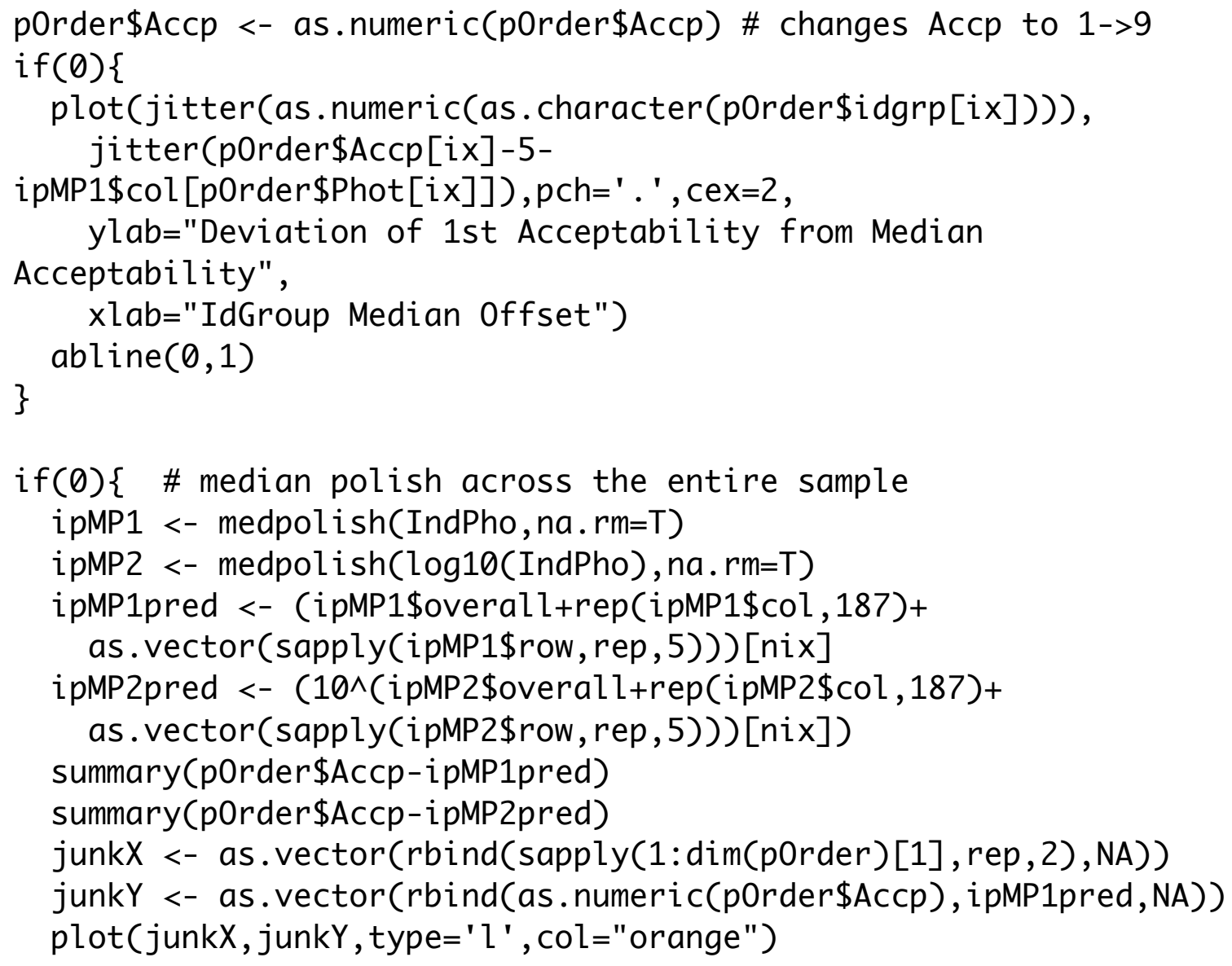


points (as . numeric(p0rder $\$$ Accp), cex $=0.5$ )

junkX <- as.vector(rbind(sapply(1: $\operatorname{dim}(p 0 r d e r)[1], r e p, 2), N A)$ )

junk $Y<-$ as.vector ( $r b i n d(a s . n u m e r i c(p 0 r d e r \$ A c c p)$, ipMP2pred, NA))

lines (junkX, junkY, type ='l' , col="light blue")

sum(abs (p0rder\$Accp-ipMP1pred)>abs (p0rder\$Accp-

ipMP2pred))/dim(p0rder)[1]

plot (ipMP1\$col)

\# pure additive is much better, create a row factor for

subsequent prediction

p0rder\$idgrp <-

as. factor (as.vector (sapply (as. factor (ipMP1\$row), rep, 5)) [nix])

\#shows that idgrp is not correlated with order

plot ( $j i t t e r(a s . n u m e r i c(p 0 r d e r \$ 0 r d r)$ ), jitter(as. numeric(p0rder $\$ i d g$

rp)), pch=' .',

xlab="Order",ylab="ID Group", cex=2)

ipMP1\$overall

ipMP1\$col

summary (as. factor(ipMP1\$row)) 


\section{Appendix C: Range Effect Data}

ID = Identification number of respondent

Range $=$ PAOT Range group (Table 3-1, p. 43)

Acceptability $=$ Acceptability rating $(-4$ to +4$)$

Photograph \# = Photograph evaluated (Figure 3-1, p. 42)

Sequence $=$ Evaluation order of photographs

\begin{tabular}{|c|c|c|c|c|}
\hline ID & Range & Acceptability & Photograph \# & Sequence \\
\hline 1 & 1 & 4 & 1 & 1 \\
\hline 1 & 1 & 2 & 2 & 2 \\
\hline 1 & 1 & 1 & 3 & 3 \\
\hline 1 & 1 & -1 & 4 & 4 \\
\hline 1 & 1 & -3 & 5 & 5 \\
\hline 2 & 1 & 4 & 1 & 1 \\
\hline 2 & 1 & -4 & 2 & 2 \\
\hline 2 & 1 & -4 & 3 & 3 \\
\hline 2 & 1 & -4 & 4 & 4 \\
\hline 2 & 1 & -4 & 5 & 5 \\
\hline 3 & 1 & 4 & 1 & 1 \\
\hline 3 & 1 & 4 & 2 & 2 \\
\hline 3 & 1 & 4 & 3 & 3 \\
\hline 3 & 1 & 4 & 4 & 4 \\
\hline 3 & 1 & 3 & 5 & 5 \\
\hline 4 & 1 & 4 & 1 & 1 \\
\hline 4 & 1 & 4 & 2 & 2 \\
\hline 4 & 1 & 4 & 3 & 3 \\
\hline 4 & 1 & 3 & 4 & 4 \\
\hline 4 & 1 & 3 & 5 & 5 \\
\hline 5 & 1 & 4 & 1 & 1 \\
\hline 5 & 1 & 2 & 2 & 2 \\
\hline 5 & 1 & 2 & 3 & 3 \\
\hline 5 & 1 & -2 & 4 & 4 \\
\hline 5 & 1 & -2 & 5 & 5 \\
\hline 6 & 1 & 4 & 1 & 1 \\
\hline 6 & 1 & 3 & 2 & 2 \\
\hline 6 & 1 & 3 & 3 & 3 \\
\hline 6 & 1 & -1 & 4 & 4 \\
\hline 6 & 1 & -3 & 5 & 5 \\
\hline
\end{tabular}




\begin{tabular}{|c|c|c|c|c|}
\hline 7 & 1 & 4 & 1 & 1 \\
\hline 7 & 1 & 3 & 2 & 2 \\
\hline 7 & 1 & 3 & 3 & 3 \\
\hline 7 & 1 & 2 & 4 & 4 \\
\hline 7 & 1 & 1 & 5 & 5 \\
\hline 8 & 1 & 4 & 1 & 1 \\
\hline 8 & 1 & 3 & 2 & 2 \\
\hline 8 & 1 & 2 & 3 & 3 \\
\hline 8 & 1 & 1 & 4 & 4 \\
\hline 8 & 1 & 1 & 5 & 5 \\
\hline 9 & 1 & 4 & 1 & 1 \\
\hline 9 & 1 & 4 & 2 & 2 \\
\hline 9 & 1 & 4 & 3 & 3 \\
\hline 9 & 1 & 2 & 4 & 4 \\
\hline 9 & 1 & 1 & 5 & 5 \\
\hline 10 & 1 & 4 & 1 & 1 \\
\hline 10 & 1 & 4 & 2 & 2 \\
\hline 10 & 1 & 3 & 3 & 3 \\
\hline 10 & 1 & 2 & 4 & 4 \\
\hline 10 & 1 & 1 & 5 & 5 \\
\hline 11 & 1 & 4 & 1 & 1 \\
\hline 11 & 1 & 4 & 2 & 2 \\
\hline 11 & 1 & 4 & 3 & 3 \\
\hline 11 & 1 & 2 & 4 & 4 \\
\hline 11 & 1 & 0 & 5 & 5 \\
\hline 12 & 1 & 4 & 1 & 1 \\
\hline 12 & 1 & 4 & 2 & 2 \\
\hline 12 & 1 & 3 & 3 & 3 \\
\hline 12 & 1 & -1 & 4 & 4 \\
\hline 12 & 1 & -2 & 5 & 5 \\
\hline 13 & 1 & 4 & 1 & 1 \\
\hline 13 & 1 & 3 & 2 & 2 \\
\hline 13 & 1 & 3 & 3 & 3 \\
\hline 13 & 1 & 2 & 4 & 4 \\
\hline 13 & 1 & 2 & 5 & 5 \\
\hline 14 & 1 & 4 & 1 & 1 \\
\hline 14 & 1 & 4 & 2 & 2 \\
\hline 14 & 1 & 3 & 3 & 3 \\
\hline 14 & 1 & 3 & 4 & 4 \\
\hline 14 & 1 & 1 & 5 & 5 \\
\hline 15 & 1 & 4 & 1 & 1 \\
\hline 15 & 1 & 3 & 2 & 2 \\
\hline 15 & 1 & 3 & 3 & 3 \\
\hline 15 & 1 & 2 & 4 & 4 \\
\hline 15 & 1 & 0 & 5 & 5 \\
\hline 16 & 1 & 4 & 1 & 1 \\
\hline 16 & 1 & 2 & 2 & 2 \\
\hline 16 & 1 & 1 & 3 & 3 \\
\hline 16 & 1 & -1 & 4 & 4 \\
\hline 16 & 1 & -3 & 5 & 5 \\
\hline 17 & 1 & 2 & 1 & 1 \\
\hline
\end{tabular}




\begin{tabular}{|c|c|c|c|c|}
\hline 17 & 1 & 3 & 2 & 2 \\
\hline 17 & 1 & 2 & 3 & 3 \\
\hline 17 & 1 & 2 & 4 & 4 \\
\hline 17 & 1 & 3 & 5 & 5 \\
\hline 18 & 1 & 4 & 1 & 1 \\
\hline 18 & 1 & 4 & 2 & 2 \\
\hline 18 & 1 & 4 & 3 & 3 \\
\hline 18 & 1 & 2 & 4 & 4 \\
\hline 18 & 1 & 1 & 5 & 5 \\
\hline 19 & 1 & 4 & 1 & 1 \\
\hline 19 & 1 & 3 & 2 & 2 \\
\hline 19 & 1 & 3 & 3 & 3 \\
\hline 19 & 1 & 1 & 4 & 4 \\
\hline 19 & 1 & -2 & 5 & 5 \\
\hline 20 & 1 & 4 & 1 & 1 \\
\hline 20 & 1 & 4 & 2 & 2 \\
\hline 20 & 1 & 4 & 3 & 3 \\
\hline 20 & 1 & 2 & 4 & 4 \\
\hline 20 & 1 & -1 & 5 & 5 \\
\hline 21 & 1 & 4 & 1 & 1 \\
\hline 21 & 1 & -2 & 2 & 2 \\
\hline 21 & 1 & -3 & 3 & 3 \\
\hline 21 & 1 & -4 & 4 & 4 \\
\hline 21 & 1 & -4 & 5 & 5 \\
\hline 22 & 1 & 4 & 1 & 1 \\
\hline 22 & 1 & 4 & 2 & 2 \\
\hline 22 & 1 & 3 & 3 & 3 \\
\hline 22 & 1 & 2 & 4 & 4 \\
\hline 22 & 1 & 2 & 5 & 5 \\
\hline 23 & 1 & 4 & 1 & 1 \\
\hline 23 & 1 & 4 & 2 & 2 \\
\hline 23 & 1 & 3 & 3 & 3 \\
\hline 23 & 1 & 3 & 4 & 4 \\
\hline 23 & 1 & 2 & 5 & 5 \\
\hline 24 & 1 & 4 & 1 & 1 \\
\hline 24 & 1 & 2 & 2 & 2 \\
\hline 24 & 1 & 1 & 3 & 3 \\
\hline 24 & 1 & 1 & 4 & 4 \\
\hline 24 & 1 & -2 & 5 & 5 \\
\hline 25 & 1 & 4 & 1 & 1 \\
\hline 25 & 1 & 3 & 2 & 2 \\
\hline 25 & 1 & 2 & 3 & 3 \\
\hline 25 & 1 & 1 & 4 & 4 \\
\hline 25 & 1 & 1 & 5 & 5 \\
\hline 26 & 1 & 4 & 1 & 1 \\
\hline 26 & 1 & 2 & 2 & 2 \\
\hline 26 & 1 & 1 & 3 & 3 \\
\hline 26 & 1 & 0 & 4 & 4 \\
\hline 26 & 1 & -2 & 5 & 5 \\
\hline 27 & 1 & 4 & 1 & 1 \\
\hline 27 & 1 & 4 & 2 & 2 \\
\hline
\end{tabular}




\begin{tabular}{|c|c|c|c|c|}
\hline 27 & 1 & 4 & 3 & 3 \\
\hline 27 & 1 & 3 & 4 & 4 \\
\hline 27 & 1 & 3 & 5 & 5 \\
\hline 28 & 1 & 4 & 1 & 1 \\
\hline 28 & 1 & 4 & 2 & 2 \\
\hline 28 & 1 & 2 & 3 & 3 \\
\hline 28 & 1 & 0 & 4 & 4 \\
\hline 28 & 1 & -3 & 5 & 5 \\
\hline 29 & 1 & 4 & 1 & 1 \\
\hline 29 & 1 & 4 & 2 & 2 \\
\hline 29 & 1 & 3 & 3 & 3 \\
\hline 29 & 1 & 3 & 4 & 4 \\
\hline 29 & 1 & 2 & 5 & 5 \\
\hline 30 & 1 & 4 & 1 & 1 \\
\hline 30 & 1 & 4 & 2 & 2 \\
\hline 30 & 1 & 4 & 3 & 3 \\
\hline 30 & 1 & 2 & 4 & 4 \\
\hline 30 & 1 & -1 & 5 & 5 \\
\hline 31 & 1 & 3 & 1 & 1 \\
\hline 31 & 1 & 2 & 2 & 2 \\
\hline 31 & 1 & -2 & 3 & 3 \\
\hline 31 & 1 & -1 & 4 & 4 \\
\hline 31 & 1 & -3 & 5 & 5 \\
\hline 32 & 1 & 4 & 1 & 1 \\
\hline 32 & 1 & 4 & 2 & 2 \\
\hline 32 & 1 & 2 & 3 & 3 \\
\hline 32 & 1 & -1 & 4 & 4 \\
\hline 32 & 1 & -4 & 5 & 5 \\
\hline 33 & 1 & 4 & 1 & 1 \\
\hline 33 & 1 & 3 & 2 & 2 \\
\hline 33 & 1 & 3 & 3 & 3 \\
\hline 33 & 1 & 3 & 4 & 4 \\
\hline 33 & 1 & 2 & 5 & 5 \\
\hline 34 & 1 & 4 & 1 & 1 \\
\hline 34 & 1 & 4 & 2 & 2 \\
\hline 34 & 1 & 2 & 3 & 3 \\
\hline 34 & 1 & 0 & 4 & 4 \\
\hline 34 & 1 & -1 & 5 & 5 \\
\hline 35 & 1 & 4 & 1 & 1 \\
\hline 35 & 1 & 3 & 2 & 2 \\
\hline 35 & 1 & 3 & 3 & 3 \\
\hline 35 & 1 & 2 & 4 & 4 \\
\hline 35 & 1 & 1 & 5 & 5 \\
\hline 36 & 1 & 4 & 1 & 1 \\
\hline 36 & 1 & 4 & 2 & 2 \\
\hline 36 & 1 & 4 & 3 & 3 \\
\hline 36 & 1 & 4 & 4 & 4 \\
\hline 36 & 1 & 4 & 5 & 5 \\
\hline 37 & 1 & 4 & 1 & 1 \\
\hline 37 & 1 & 2 & 2 & 2 \\
\hline 37 & 1 & -1 & 3 & 3 \\
\hline
\end{tabular}




\begin{tabular}{|c|c|c|c|c|}
\hline 37 & 1 & -3 & 4 & 4 \\
\hline 37 & 1 & -4 & 5 & 5 \\
\hline 38 & 1 & 4 & 1 & 1 \\
\hline 38 & 1 & 4 & 2 & 2 \\
\hline 38 & 1 & 3 & 3 & 3 \\
\hline 38 & 1 & 3 & 4 & 4 \\
\hline 38 & 1 & 1 & 5 & 5 \\
\hline 39 & 1 & 4 & 1 & 1 \\
\hline 39 & 1 & 2 & 2 & 2 \\
\hline 39 & 1 & 2 & 3 & 3 \\
\hline 39 & 1 & 0 & 4 & 4 \\
\hline 39 & 1 & 0 & 5 & 5 \\
\hline 40 & 1 & 4 & 1 & 1 \\
\hline 40 & 1 & 4 & 2 & 2 \\
\hline 40 & 1 & 4 & 3 & 3 \\
\hline 40 & 1 & 4 & 4 & 4 \\
\hline 40 & 1 & 4 & 5 & 5 \\
\hline 41 & 1 & 4 & 1 & 1 \\
\hline 41 & 1 & 4 & 2 & 2 \\
\hline 41 & 1 & 3 & 3 & 3 \\
\hline 41 & 1 & 3 & 4 & 4 \\
\hline 41 & 1 & 1 & 5 & 5 \\
\hline 42 & 1 & 4 & 1 & 1 \\
\hline 42 & 1 & 4 & 2 & 2 \\
\hline 42 & 1 & 3 & 3 & 3 \\
\hline 42 & 1 & 2 & 4 & 4 \\
\hline 42 & 1 & -1 & 5 & 5 \\
\hline 43 & 1 & 4 & 1 & 1 \\
\hline 43 & 1 & 3 & 2 & 2 \\
\hline 43 & 1 & 3 & 3 & 3 \\
\hline 43 & 1 & 3 & 4 & 4 \\
\hline 43 & 1 & 2 & 5 & 5 \\
\hline 44 & 1 & 4 & 1 & 1 \\
\hline 44 & 1 & 2 & 2 & 2 \\
\hline 44 & 1 & -2 & 3 & 3 \\
\hline 44 & 1 & -4 & 4 & 4 \\
\hline 44 & 1 & -4 & 5 & 5 \\
\hline 45 & 1 & 4 & 1 & 1 \\
\hline 45 & 1 & 2 & 2 & 2 \\
\hline 45 & 1 & 0 & 3 & 3 \\
\hline 45 & 1 & -3 & 4 & 4 \\
\hline 45 & 1 & -4 & 5 & 5 \\
\hline 46 & 1 & 4 & 1 & 1 \\
\hline 46 & 1 & 3 & 2 & 2 \\
\hline 46 & 1 & 3 & 3 & 3 \\
\hline 46 & 1 & 2 & 4 & 4 \\
\hline 46 & 1 & 1 & 5 & 5 \\
\hline 47 & 1 & 4 & 1 & 1 \\
\hline 47 & 1 & 4 & 2 & 2 \\
\hline 47 & 1 & 4 & 3 & 3 \\
\hline 47 & 1 & 4 & 4 & 4 \\
\hline
\end{tabular}




\begin{tabular}{|c|c|c|c|c|}
\hline 47 & 1 & 3 & 5 & 5 \\
\hline 48 & 1 & 4 & 1 & 1 \\
\hline 48 & 1 & 3 & 2 & 2 \\
\hline 48 & 1 & 1 & 3 & 3 \\
\hline 48 & 1 & -2 & 4 & 4 \\
\hline 48 & 1 & -3 & 5 & 5 \\
\hline 49 & 1 & 4 & 1 & 1 \\
\hline 49 & 1 & 2 & 2 & 2 \\
\hline 49 & 1 & 0 & 3 & 3 \\
\hline 49 & 1 & -1 & 4 & 4 \\
\hline 49 & 1 & -3 & 5 & 5 \\
\hline 50 & 1 & 4 & 1 & 1 \\
\hline 50 & 1 & 3 & 2 & 2 \\
\hline 50 & 1 & 1 & 3 & 3 \\
\hline 50 & 1 & -1 & 4 & 4 \\
\hline 50 & 1 & -3 & 5 & 5 \\
\hline 51 & 1 & 4 & 1 & 1 \\
\hline 51 & 1 & 3 & 2 & 2 \\
\hline 51 & 1 & 2 & 3 & 3 \\
\hline 51 & 1 & -2 & 4 & 4 \\
\hline 51 & 1 & -3 & 5 & 5 \\
\hline 52 & 1 & 4 & 1 & 1 \\
\hline 52 & 1 & 3 & 2 & 2 \\
\hline 52 & 1 & 3 & 3 & 3 \\
\hline 52 & 1 & 2 & 4 & 4 \\
\hline 52 & 1 & -1 & 5 & 5 \\
\hline 53 & 1 & 4 & 1 & 1 \\
\hline 53 & 1 & 2 & 2 & 2 \\
\hline 53 & 1 & 0 & 3 & 3 \\
\hline 53 & 1 & -2 & 4 & 4 \\
\hline 53 & 1 & -3 & 5 & 5 \\
\hline 54 & 1 & -3 & 1 & 1 \\
\hline 54 & 1 & -3 & 2 & 2 \\
\hline 54 & 1 & -4 & 3 & 3 \\
\hline 54 & 1 & -4 & 4 & 4 \\
\hline 54 & 1 & -4 & 5 & 5 \\
\hline 55 & 1 & 4 & 1 & 1 \\
\hline 55 & 1 & 3 & 2 & 2 \\
\hline 55 & 1 & 3 & 3 & 3 \\
\hline 55 & 1 & 2 & 4 & 4 \\
\hline 55 & 1 & -1 & 5 & 5 \\
\hline 56 & 1 & 4 & 1 & 1 \\
\hline 56 & 1 & 3 & 2 & 2 \\
\hline 56 & 1 & 1 & 3 & 3 \\
\hline 56 & 1 & -1 & 4 & 4 \\
\hline 56 & 1 & -2 & 5 & 5 \\
\hline 57 & 1 & 4 & 1 & 1 \\
\hline 57 & 1 & 2 & 2 & 2 \\
\hline 57 & 1 & 0 & 3 & 3 \\
\hline 57 & 1 & -2 & 4 & 4 \\
\hline 57 & 1 & -4 & 5 & 5 \\
\hline
\end{tabular}




\begin{tabular}{|c|c|c|c|c|}
\hline 58 & 1 & 4 & 1 & 1 \\
\hline 58 & 1 & 3 & 2 & 2 \\
\hline 58 & 1 & 2 & 3 & 3 \\
\hline 58 & 1 & 1 & 4 & 4 \\
\hline 58 & 1 & 0 & 5 & 5 \\
\hline 59 & 1 & 4 & 1 & 1 \\
\hline 59 & 1 & 4 & 2 & 2 \\
\hline 59 & 1 & 4 & 3 & 3 \\
\hline 59 & 1 & 3 & 4 & 4 \\
\hline 59 & 1 & 3 & 5 & 5 \\
\hline 60 & 1 & 4 & 1 & 1 \\
\hline 60 & 1 & 4 & 2 & 2 \\
\hline 60 & 1 & 4 & 3 & 3 \\
\hline 60 & 1 & 3 & 4 & 4 \\
\hline 60 & 1 & 2 & 5 & 5 \\
\hline 61 & 1 & 4 & 1 & 1 \\
\hline 61 & 1 & 2 & 2 & 2 \\
\hline 61 & 1 & 1 & 3 & 3 \\
\hline 61 & 1 & -2 & 4 & 4 \\
\hline 61 & 1 & -3 & 5 & 5 \\
\hline 62 & 1 & 3 & 1 & 1 \\
\hline 62 & 1 & 3 & 2 & 2 \\
\hline 62 & 1 & 3 & 3 & 3 \\
\hline 62 & 1 & 2 & 4 & 4 \\
\hline 62 & 1 & 1 & 5 & 5 \\
\hline 63 & 1 & 4 & 1 & 1 \\
\hline 63 & 1 & 3 & 2 & 2 \\
\hline 63 & 1 & 1 & 3 & 3 \\
\hline 63 & 1 & 0 & 4 & 4 \\
\hline 63 & 1 & -2 & 5 & 5 \\
\hline 64 & 1 & 4 & 1 & 1 \\
\hline 64 & 1 & 3 & 2 & 2 \\
\hline 64 & 1 & 2 & 3 & 3 \\
\hline 64 & 1 & -1 & 4 & 4 \\
\hline 64 & 1 & -2 & 5 & 5 \\
\hline 65 & 1 & 4 & 1 & 1 \\
\hline 65 & 1 & 3 & 2 & 2 \\
\hline 65 & 1 & 2 & 3 & 3 \\
\hline 65 & 1 & 1 & 4 & 4 \\
\hline 65 & 1 & -1 & 5 & 5 \\
\hline 66 & 1 & 4 & 1 & 1 \\
\hline 66 & 1 & 3 & 2 & 2 \\
\hline 66 & 1 & 2 & 3 & 3 \\
\hline 66 & 1 & -1 & 4 & 4 \\
\hline 66 & 1 & -4 & 5 & 5 \\
\hline 67 & 1 & 4 & 1 & 1 \\
\hline 67 & 1 & 4 & 2 & 2 \\
\hline 67 & 1 & 4 & 3 & 3 \\
\hline 67 & 1 & 3 & 4 & 4 \\
\hline 67 & 1 & -2 & 5 & 5 \\
\hline 68 & 1 & 4 & 1 & 1 \\
\hline
\end{tabular}




\begin{tabular}{|c|c|c|c|c|}
\hline 68 & 1 & 3 & 2 & 2 \\
\hline 68 & 1 & 1 & 3 & 3 \\
\hline 68 & 1 & -4 & 4 & 4 \\
\hline 68 & 1 & -4 & 5 & 5 \\
\hline 69 & 1 & 0 & 1 & 1 \\
\hline 69 & 1 & 1 & 2 & 2 \\
\hline 69 & 1 & 1 & 3 & 3 \\
\hline 69 & 1 & 1 & 4 & 4 \\
\hline 69 & 1 & 1 & 5 & 5 \\
\hline 70 & 1 & 4 & 1 & 1 \\
\hline 70 & 1 & 3 & 2 & 2 \\
\hline 70 & 1 & 3 & 3 & 3 \\
\hline 70 & 1 & 2 & 4 & 4 \\
\hline 70 & 1 & -1 & 5 & 5 \\
\hline 71 & 1 & 4 & 1 & 1 \\
\hline 71 & 1 & 2 & 2 & 2 \\
\hline 71 & 1 & 1 & 3 & 3 \\
\hline 71 & 1 & -1 & 4 & 4 \\
\hline 71 & 1 & -2 & 5 & 5 \\
\hline 72 & 1 & 4 & 1 & 1 \\
\hline 72 & 1 & 4 & 2 & 2 \\
\hline 72 & 1 & 3 & 3 & 3 \\
\hline 72 & 1 & 2 & 4 & 4 \\
\hline 72 & 1 & -1 & 5 & 5 \\
\hline 73 & 1 & 4 & 1 & 1 \\
\hline 73 & 1 & 2 & 2 & 2 \\
\hline 73 & 1 & -1 & 3 & 3 \\
\hline 73 & 1 & -3 & 4 & 4 \\
\hline 73 & 1 & -4 & 5 & 5 \\
\hline 74 & 1 & 4 & 1 & 1 \\
\hline 74 & 1 & 4 & 2 & 2 \\
\hline 74 & 1 & 4 & 3 & 3 \\
\hline 74 & 1 & 3 & 4 & 4 \\
\hline 74 & 1 & 3 & 5 & 5 \\
\hline 75 & 1 & 4 & 1 & 1 \\
\hline 75 & 1 & 2 & 2 & 2 \\
\hline 75 & 1 & -1 & 3 & 3 \\
\hline 75 & 1 & -3 & 4 & 4 \\
\hline 75 & 1 & -4 & 5 & 5 \\
\hline 76 & 1 & 4 & 1 & 1 \\
\hline 76 & 1 & 4 & 2 & 2 \\
\hline 76 & 1 & 3 & 3 & 3 \\
\hline 76 & 1 & 2 & 4 & 4 \\
\hline 76 & 1 & 2 & 5 & 5 \\
\hline 77 & 1 & 4 & 1 & 1 \\
\hline 77 & 1 & 2 & 2 & 2 \\
\hline 77 & 1 & 2 & 3 & 3 \\
\hline 77 & 1 & 1 & 4 & 4 \\
\hline 77 & 1 & -1 & 5 & 5 \\
\hline 78 & 1 & 4 & 1 & 1 \\
\hline 78 & 1 & 3 & 2 & 2 \\
\hline
\end{tabular}




\begin{tabular}{|c|c|c|c|c|}
\hline 78 & 1 & 3 & 3 & 3 \\
\hline 78 & 1 & 1 & 4 & 4 \\
\hline 78 & 1 & -1 & 5 & 5 \\
\hline 79 & 1 & 4 & 1 & 1 \\
\hline 79 & 1 & 3 & 2 & 2 \\
\hline 79 & 1 & 2 & 3 & 3 \\
\hline 79 & 1 & 1 & 4 & 4 \\
\hline 79 & 1 & -2 & 5 & 5 \\
\hline 80 & 1 & 4 & 1 & 1 \\
\hline 80 & 1 & 3 & 2 & 2 \\
\hline 80 & 1 & 3 & 3 & 3 \\
\hline 80 & 1 & 2 & 4 & 4 \\
\hline 80 & 1 & 1 & 5 & 5 \\
\hline 81 & 1 & 4 & 1 & 1 \\
\hline 81 & 1 & 4 & 2 & 2 \\
\hline 81 & 1 & 3 & 3 & 3 \\
\hline 81 & 1 & 1 & 4 & 4 \\
\hline 81 & 1 & 0 & 5 & 5 \\
\hline 82 & 1 & 4 & 1 & 1 \\
\hline 82 & 1 & 3 & 2 & 2 \\
\hline 82 & 1 & 2 & 3 & 3 \\
\hline 82 & 1 & 2 & 4 & 4 \\
\hline 82 & 1 & 1 & 5 & 5 \\
\hline 83 & 1 & 4 & 1 & 1 \\
\hline 83 & 1 & 4 & 2 & 2 \\
\hline 83 & 1 & 2 & 3 & 3 \\
\hline 83 & 1 & 0 & 4 & 4 \\
\hline 83 & 1 & -2 & 5 & 5 \\
\hline 84 & 1 & 4 & 1 & 1 \\
\hline 84 & 1 & 3 & 2 & 2 \\
\hline 84 & 1 & 3 & 3 & 3 \\
\hline 84 & 1 & 3 & 4 & 4 \\
\hline 84 & 1 & 2 & 5 & 5 \\
\hline 85 & 1 & 4 & 1 & 1 \\
\hline 85 & 1 & 4 & 2 & 2 \\
\hline 85 & 1 & 3 & 3 & 3 \\
\hline 85 & 1 & 2 & 4 & 4 \\
\hline 85 & 1 & -1 & 5 & 5 \\
\hline 86 & 1 & 4 & 1 & 1 \\
\hline 86 & 1 & 1 & 2 & 2 \\
\hline 86 & 1 & -1 & 3 & 3 \\
\hline 86 & 1 & -2 & 4 & 4 \\
\hline 86 & 1 & -4 & 5 & 5 \\
\hline 87 & 1 & 4 & 1 & 1 \\
\hline 87 & 1 & 4 & 2 & 2 \\
\hline 87 & 1 & 3 & 3 & 3 \\
\hline 87 & 1 & 2 & 4 & 4 \\
\hline 87 & 1 & -2 & 5 & 5 \\
\hline 88 & 1 & 4 & 1 & 1 \\
\hline 88 & 1 & 3 & 2 & 2 \\
\hline 88 & 1 & 3 & 3 & 3 \\
\hline
\end{tabular}




\begin{tabular}{|c|c|c|c|c|}
\hline 88 & 1 & 2 & 4 & 4 \\
\hline 88 & 1 & 0 & 5 & 5 \\
\hline 89 & 1 & 4 & 1 & 1 \\
\hline 89 & 1 & 3 & 2 & 2 \\
\hline 89 & 1 & 2 & 3 & 3 \\
\hline 89 & 1 & -1 & 4 & 4 \\
\hline 89 & 1 & -3 & 5 & 5 \\
\hline 90 & 1 & 4 & 1 & 1 \\
\hline 90 & 1 & 3 & 2 & 2 \\
\hline 90 & 1 & 2 & 3 & 3 \\
\hline 90 & 1 & -1 & 4 & 4 \\
\hline 90 & 1 & -4 & 5 & 5 \\
\hline 91 & 1 & 4 & 1 & 1 \\
\hline 91 & 1 & 1 & 2 & 2 \\
\hline 91 & 1 & -1 & 3 & 3 \\
\hline 91 & 1 & -3 & 4 & 4 \\
\hline 91 & 1 & -4 & 5 & 5 \\
\hline 92 & 1 & 4 & 1 & 1 \\
\hline 92 & 1 & 1 & 2 & 2 \\
\hline 92 & 1 & 1 & 3 & 3 \\
\hline 92 & 1 & -2 & 4 & 4 \\
\hline 92 & 1 & -4 & 5 & 5 \\
\hline 93 & 1 & 4 & 1 & 1 \\
\hline 93 & 1 & 1 & 2 & 2 \\
\hline 93 & 1 & 1 & 3 & 3 \\
\hline 93 & 1 & -2 & 4 & 4 \\
\hline 93 & 1 & -3 & 5 & 5 \\
\hline 94 & 1 & 1 & 1 & 1 \\
\hline 94 & 1 & 1 & 2 & 2 \\
\hline 94 & 1 & 0 & 3 & 3 \\
\hline 94 & 1 & -1 & 4 & 4 \\
\hline 94 & 1 & -1 & 5 & 5 \\
\hline 95 & 1 & 4 & 1 & 1 \\
\hline 95 & 1 & 2 & 2 & 2 \\
\hline 95 & 1 & 1 & 3 & 3 \\
\hline 95 & 1 & -1 & 4 & 4 \\
\hline 95 & 1 & -3 & 5 & 5 \\
\hline 96 & 1 & 4 & 1 & 1 \\
\hline 96 & 1 & 3 & 2 & 2 \\
\hline 96 & 1 & 3 & 3 & 3 \\
\hline 96 & 1 & 1 & 4 & 4 \\
\hline 96 & 1 & -2 & 5 & 5 \\
\hline 97 & 1 & 4 & 1 & 1 \\
\hline 97 & 1 & 2 & 2 & 2 \\
\hline 97 & 1 & 1 & 3 & 3 \\
\hline 97 & 1 & -1 & 4 & 4 \\
\hline 97 & 1 & -4 & 5 & 5 \\
\hline 98 & 1 & 4 & 1 & 1 \\
\hline 98 & 1 & 4 & 2 & 2 \\
\hline 98 & 1 & 3 & 3 & 3 \\
\hline 98 & 1 & 3 & 4 & 4 \\
\hline
\end{tabular}




\begin{tabular}{|c|c|c|c|c|}
\hline 98 & 1 & 1 & 5 & 5 \\
\hline 99 & 1 & 4 & 1 & 1 \\
\hline 99 & 1 & 3 & 2 & 2 \\
\hline 99 & 1 & 3 & 3 & 3 \\
\hline 99 & 1 & 1 & 4 & 4 \\
\hline 99 & 1 & -2 & 5 & 5 \\
\hline 100 & 1 & 4 & 1 & 1 \\
\hline 100 & 1 & 2 & 2 & 2 \\
\hline 100 & 1 & 2 & 3 & 3 \\
\hline 100 & 1 & -1 & 4 & 4 \\
\hline 100 & 1 & -3 & 5 & 5 \\
\hline 101 & 2 & -1 & 6 & 1 \\
\hline 101 & 2 & -2 & 7 & 2 \\
\hline 101 & 2 & -2 & 8 & 3 \\
\hline 101 & 2 & -2 & 9 & 4 \\
\hline 101 & 2 & -2 & 10 & 5 \\
\hline 102 & 2 & 3 & 6 & 1 \\
\hline 102 & 2 & 3 & 7 & 2 \\
\hline 102 & 2 & 2 & 8 & 3 \\
\hline 102 & 2 & 2 & 9 & 4 \\
\hline 102 & 2 & -1 & 10 & 5 \\
\hline 103 & 2 & -3 & 6 & 1 \\
\hline 103 & 2 & -3 & 7 & 2 \\
\hline 103 & 2 & -3 & 8 & 3 \\
\hline 103 & 2 & -4 & 9 & 4 \\
\hline 103 & 2 & -4 & 10 & 5 \\
\hline 104 & 2 & 3 & 6 & 1 \\
\hline 104 & 2 & 2 & 7 & 2 \\
\hline 104 & 2 & 1 & 8 & 3 \\
\hline 104 & 2 & 0 & 9 & 4 \\
\hline 104 & 2 & -1 & 10 & 5 \\
\hline 105 & 2 & 1 & 6 & 1 \\
\hline 105 & 2 & 0 & 7 & 2 \\
\hline 105 & 2 & -1 & 8 & 3 \\
\hline 105 & 2 & -1 & 9 & 4 \\
\hline 105 & 2 & -2 & 10 & 5 \\
\hline 106 & 2 & 4 & 6 & 1 \\
\hline 106 & 2 & 2 & 7 & 2 \\
\hline 106 & 2 & 1 & 8 & 3 \\
\hline 106 & 2 & 1 & 9 & 4 \\
\hline 106 & 2 & -1 & 10 & 5 \\
\hline 107 & 2 & 4 & 6 & 1 \\
\hline 107 & 2 & 3 & 7 & 2 \\
\hline 107 & 2 & 1 & 8 & 3 \\
\hline 107 & 2 & -1 & 9 & 4 \\
\hline 107 & 2 & -2 & 10 & 5 \\
\hline 108 & 2 & -1 & 6 & 1 \\
\hline 108 & 2 & -2 & 7 & 2 \\
\hline 108 & 2 & -3 & 8 & 3 \\
\hline 108 & 2 & -4 & 9 & 4 \\
\hline 108 & 2 & -4 & 10 & 5 \\
\hline
\end{tabular}




\begin{tabular}{|c|c|c|c|c|}
\hline 109 & 2 & -2 & 6 & 1 \\
\hline 109 & 2 & -2 & 7 & 2 \\
\hline 109 & 2 & -3 & 8 & 3 \\
\hline 109 & 2 & -4 & 9 & 4 \\
\hline 109 & 2 & -4 & 10 & 5 \\
\hline 110 & 2 & 2 & 6 & 1 \\
\hline 110 & 2 & 2 & 7 & 2 \\
\hline 110 & 2 & 2 & 8 & 3 \\
\hline 110 & 2 & 1 & 9 & 4 \\
\hline 110 & 2 & 0 & 10 & 5 \\
\hline 111 & 2 & -3 & 6 & 1 \\
\hline 111 & 2 & -3 & 7 & 2 \\
\hline 111 & 2 & -3 & 8 & 3 \\
\hline 111 & 2 & -3 & 9 & 4 \\
\hline 111 & 2 & -4 & 10 & 5 \\
\hline 112 & 2 & -2 & 6 & 1 \\
\hline 112 & 2 & -3 & 7 & 2 \\
\hline 112 & 2 & -4 & 8 & 3 \\
\hline 112 & 2 & -4 & 9 & 4 \\
\hline 112 & 2 & -4 & 10 & 5 \\
\hline 113 & 2 & -1 & 6 & 1 \\
\hline 113 & 2 & -2 & 7 & 2 \\
\hline 113 & 2 & -2 & 8 & 3 \\
\hline 113 & 2 & -3 & 9 & 4 \\
\hline 113 & 2 & -4 & 10 & 5 \\
\hline 114 & 2 & -1 & 6 & 1 \\
\hline 114 & 2 & -1 & 7 & 2 \\
\hline 114 & 2 & -2 & 8 & 3 \\
\hline 114 & 2 & -2 & 9 & 4 \\
\hline 114 & 2 & -3 & 10 & 5 \\
\hline 115 & 2 & 2 & 6 & 1 \\
\hline 115 & 2 & 1 & 7 & 2 \\
\hline 115 & 2 & 1 & 8 & 3 \\
\hline 115 & 2 & 0 & 9 & 4 \\
\hline 115 & 2 & -1 & 10 & 5 \\
\hline 116 & 2 & 0 & 6 & 1 \\
\hline 116 & 2 & -1 & 7 & 2 \\
\hline 116 & 2 & -2 & 8 & 3 \\
\hline 116 & 2 & -3 & 9 & 4 \\
\hline 116 & 2 & -4 & 10 & 5 \\
\hline 117 & 2 & -1 & 6 & 1 \\
\hline 117 & 2 & -2 & 7 & 2 \\
\hline 117 & 2 & -2 & 8 & 3 \\
\hline 117 & 2 & -3 & 9 & 4 \\
\hline 117 & 2 & -3 & 10 & 5 \\
\hline 118 & 2 & -2 & 6 & 1 \\
\hline 118 & 2 & -3 & 7 & 2 \\
\hline 118 & 2 & -3 & 8 & 3 \\
\hline 118 & 2 & -3 & 9 & 4 \\
\hline 118 & 2 & -4 & 10 & 5 \\
\hline 119 & 2 & -1 & 6 & 1 \\
\hline
\end{tabular}




\begin{tabular}{|c|c|c|c|c|}
\hline 119 & 2 & -4 & 7 & 2 \\
\hline 119 & 2 & -2 & 8 & 3 \\
\hline 119 & 2 & -2 & 9 & 4 \\
\hline 119 & 2 & -3 & 10 & 5 \\
\hline 120 & 2 & 2 & 6 & 1 \\
\hline 120 & 2 & 1 & 7 & 2 \\
\hline 120 & 2 & 1 & 8 & 3 \\
\hline 120 & 2 & 0 & 9 & 4 \\
\hline 120 & 2 & -2 & 10 & 5 \\
\hline 121 & 2 & -2 & 6 & 1 \\
\hline 121 & 2 & -2 & 7 & 2 \\
\hline 121 & 2 & -3 & 8 & 3 \\
\hline 121 & 2 & -3 & 9 & 4 \\
\hline 121 & 2 & -4 & 10 & 5 \\
\hline 122 & 2 & 1 & 6 & 1 \\
\hline 122 & 2 & 1 & 7 & 2 \\
\hline 122 & 2 & 0 & 8 & 3 \\
\hline 122 & 2 & -1 & 9 & 4 \\
\hline 122 & 2 & -2 & 10 & 5 \\
\hline 123 & 2 & 3 & 6 & 1 \\
\hline 123 & 2 & 2 & 7 & 2 \\
\hline 123 & 2 & 2 & 8 & 3 \\
\hline 123 & 2 & 0 & 9 & 4 \\
\hline 123 & 2 & 0 & 10 & 5 \\
\hline 124 & 2 & 3 & 6 & 1 \\
\hline 124 & 2 & 3 & 7 & 2 \\
\hline 124 & 2 & 3 & 8 & 3 \\
\hline 124 & 2 & 2 & 9 & 4 \\
\hline 124 & 2 & 2 & 10 & 5 \\
\hline 125 & 2 & 4 & 6 & 1 \\
\hline 125 & 2 & 3 & 7 & 2 \\
\hline 125 & 2 & 3 & 8 & 3 \\
\hline 125 & 2 & 2 & 9 & 4 \\
\hline 125 & 2 & 2 & 10 & 5 \\
\hline 126 & 2 & 3 & 6 & 1 \\
\hline 126 & 2 & 2 & 7 & 2 \\
\hline 126 & 2 & -1 & 8 & 3 \\
\hline 126 & 2 & -2 & 9 & 4 \\
\hline 126 & 2 & -2 & 10 & 5 \\
\hline 127 & 2 & 0 & 6 & 1 \\
\hline 127 & 2 & -1 & 7 & 2 \\
\hline 127 & 2 & -1 & 8 & 3 \\
\hline 127 & 2 & -1 & 9 & 4 \\
\hline 127 & 2 & -2 & 10 & 5 \\
\hline 128 & 2 & -2 & 6 & 1 \\
\hline 128 & 2 & -2 & 7 & 2 \\
\hline 128 & 2 & -3 & 8 & 3 \\
\hline 128 & 2 & -3 & 9 & 4 \\
\hline 128 & 2 & -4 & 10 & 5 \\
\hline 129 & 2 & 4 & 6 & 1 \\
\hline 129 & 2 & 3 & 7 & 2 \\
\hline
\end{tabular}




\begin{tabular}{|c|c|c|c|c|}
\hline 129 & 2 & 2 & 8 & 3 \\
\hline 129 & 2 & 2 & 9 & 4 \\
\hline 129 & 2 & 1 & 10 & 5 \\
\hline 130 & 2 & 2 & 6 & 1 \\
\hline 130 & 2 & 2 & 7 & 2 \\
\hline 130 & 2 & 2 & 8 & 3 \\
\hline 130 & 2 & 2 & 9 & 4 \\
\hline 130 & 2 & 0 & 10 & 5 \\
\hline 131 & 2 & -1 & 6 & 1 \\
\hline 131 & 2 & -2 & 7 & 2 \\
\hline 131 & 2 & -2 & 8 & 3 \\
\hline 131 & 2 & -3 & 9 & 4 \\
\hline 131 & 2 & -3 & 10 & 5 \\
\hline 132 & 2 & 1 & 6 & 1 \\
\hline 132 & 2 & 0 & 7 & 2 \\
\hline 132 & 2 & 0 & 8 & 3 \\
\hline 132 & 2 & -2 & 9 & 4 \\
\hline 132 & 2 & -3 & 10 & 5 \\
\hline 133 & 2 & 4 & 6 & 1 \\
\hline 133 & 2 & 4 & 7 & 2 \\
\hline 133 & 2 & 4 & 8 & 3 \\
\hline 133 & 2 & 3 & 9 & 4 \\
\hline 133 & 2 & 3 & 10 & 5 \\
\hline 134 & 2 & -1 & 6 & 1 \\
\hline 134 & 2 & -1 & 7 & 2 \\
\hline 134 & 2 & -1 & 8 & 3 \\
\hline 134 & 2 & -1 & 9 & 4 \\
\hline 134 & 2 & -2 & 10 & 5 \\
\hline 135 & 2 & -4 & 6 & 1 \\
\hline 135 & 2 & -2 & 7 & 2 \\
\hline 135 & 2 & -1 & 8 & 3 \\
\hline 135 & 2 & 1 & 9 & 4 \\
\hline 135 & 2 & 1 & 10 & 5 \\
\hline 136 & 2 & -2 & 6 & 1 \\
\hline 136 & 2 & -2 & 7 & 2 \\
\hline 136 & 2 & -3 & 8 & 3 \\
\hline 136 & 2 & -3 & 9 & 4 \\
\hline 136 & 2 & -4 & 10 & 5 \\
\hline 137 & 3 & 2 & 11 & 1 \\
\hline 137 & 3 & 2 & 12 & 2 \\
\hline 137 & 3 & 1 & 13 & 3 \\
\hline 137 & 3 & 1 & 14 & 4 \\
\hline 137 & 3 & 0 & 15 & 5 \\
\hline 138 & 3 & -2 & 11 & 1 \\
\hline 138 & 3 & -2 & 12 & 2 \\
\hline 138 & 3 & -3 & 13 & 3 \\
\hline 138 & 3 & -3 & 14 & 4 \\
\hline 138 & 3 & -4 & 15 & 5 \\
\hline 139 & 3 & -4 & 11 & 1 \\
\hline 139 & 3 & -4 & 12 & 2 \\
\hline 139 & 3 & -4 & 13 & 3 \\
\hline
\end{tabular}




\begin{tabular}{|c|c|c|c|c|}
\hline 139 & 3 & -4 & 14 & 4 \\
\hline 139 & 3 & -4 & 15 & 5 \\
\hline 140 & 3 & -3 & 11 & 1 \\
\hline 140 & 3 & -4 & 12 & 2 \\
\hline 140 & 3 & -4 & 13 & 3 \\
\hline 140 & 3 & -4 & 14 & 4 \\
\hline 140 & 3 & -4 & 15 & 5 \\
\hline 141 & 3 & 1 & 11 & 1 \\
\hline 141 & 3 & -1 & 12 & 2 \\
\hline 141 & 3 & -2 & 13 & 3 \\
\hline 141 & 3 & -3 & 14 & 4 \\
\hline 141 & 3 & -3 & 15 & 5 \\
\hline 142 & 3 & 2 & 11 & 1 \\
\hline 142 & 3 & 1 & 12 & 2 \\
\hline 142 & 3 & 0 & 13 & 3 \\
\hline 142 & 3 & 0 & 14 & 4 \\
\hline 142 & 3 & -1 & 15 & 5 \\
\hline 143 & 3 & 0 & 11 & 1 \\
\hline 143 & 3 & 0 & 12 & 2 \\
\hline 143 & 3 & -1 & 13 & 3 \\
\hline 143 & 3 & -1 & 14 & 4 \\
\hline 143 & 3 & -1 & 15 & 5 \\
\hline 144 & 3 & 1 & 11 & 1 \\
\hline 144 & 3 & 1 & 12 & 2 \\
\hline 144 & 3 & 0 & 13 & 3 \\
\hline 144 & 3 & 0 & 14 & 4 \\
\hline 144 & 3 & -3 & 15 & 5 \\
\hline 145 & 3 & 1 & 11 & 1 \\
\hline 145 & 3 & -1 & 12 & 2 \\
\hline 145 & 3 & -2 & 13 & 3 \\
\hline 145 & 3 & -2 & 14 & 4 \\
\hline 145 & 3 & -3 & 15 & 5 \\
\hline 146 & 3 & 0 & 11 & 1 \\
\hline 146 & 3 & -1 & 12 & 2 \\
\hline 146 & 3 & -2 & 13 & 3 \\
\hline 146 & 3 & -2 & 14 & 4 \\
\hline 146 & 3 & -2 & 15 & 5 \\
\hline 147 & 3 & 1 & 11 & 1 \\
\hline 147 & 3 & -1 & 12 & 2 \\
\hline 147 & 3 & -2 & 13 & 3 \\
\hline 147 & 3 & -3 & 14 & 4 \\
\hline 147 & 3 & -4 & 15 & 5 \\
\hline 148 & 3 & -3 & 11 & 1 \\
\hline 148 & 3 & -3 & 12 & 2 \\
\hline 148 & 3 & -3 & 13 & 3 \\
\hline 148 & 3 & -3 & 14 & 4 \\
\hline 148 & 3 & -3 & 15 & 5 \\
\hline 149 & 3 & -3 & 11 & 1 \\
\hline 149 & 3 & -3 & 12 & 2 \\
\hline 149 & 3 & -4 & 13 & 3 \\
\hline 149 & 3 & -4 & 14 & 4 \\
\hline
\end{tabular}




\begin{tabular}{|c|c|c|c|c|}
\hline 149 & 3 & -4 & 15 & 5 \\
\hline 150 & 3 & -2 & 11 & 1 \\
\hline 150 & 3 & -2 & 12 & 2 \\
\hline 150 & 3 & -3 & 13 & 3 \\
\hline 150 & 3 & -3 & 14 & 4 \\
\hline 150 & 3 & -3 & 15 & 5 \\
\hline 151 & 3 & -3 & 11 & 1 \\
\hline 151 & 3 & -3 & 12 & 2 \\
\hline 151 & 3 & -4 & 13 & 3 \\
\hline 151 & 3 & -4 & 14 & 4 \\
\hline 151 & 3 & -4 & 15 & 5 \\
\hline 152 & 3 & -2 & 11 & 1 \\
\hline 152 & 3 & -3 & 12 & 2 \\
\hline 152 & 3 & -3 & 13 & 3 \\
\hline 152 & 3 & -3 & 14 & 4 \\
\hline 152 & 3 & -4 & 15 & 5 \\
\hline 153 & 3 & -3 & 11 & 1 \\
\hline 153 & 3 & -4 & 12 & 2 \\
\hline 153 & 3 & -4 & 13 & 3 \\
\hline 153 & 3 & -4 & 14 & 4 \\
\hline 153 & 3 & -4 & 15 & 5 \\
\hline 154 & 3 & 3 & 11 & 1 \\
\hline 154 & 3 & 1 & 12 & 2 \\
\hline 154 & 3 & 1 & 13 & 3 \\
\hline 154 & 3 & -1 & 14 & 4 \\
\hline 154 & 3 & -2 & 15 & 5 \\
\hline 155 & 3 & -3 & 11 & 1 \\
\hline 155 & 3 & -3 & 12 & 2 \\
\hline 155 & 3 & -3 & 13 & 3 \\
\hline 155 & 3 & -4 & 14 & 4 \\
\hline 155 & 3 & -4 & 15 & 5 \\
\hline 156 & 3 & -3 & 11 & 1 \\
\hline 156 & 3 & -3 & 12 & 2 \\
\hline 156 & 3 & -3 & 13 & 3 \\
\hline 156 & 3 & -4 & 14 & 4 \\
\hline 156 & 3 & -4 & 15 & 5 \\
\hline 157 & 3 & -2 & 11 & 1 \\
\hline 157 & 3 & -2 & 12 & 2 \\
\hline 157 & 3 & -2 & 13 & 3 \\
\hline 157 & 3 & -2 & 14 & 4 \\
\hline 157 & 3 & -2 & 15 & 5 \\
\hline 158 & 3 & -3 & 11 & 1 \\
\hline 158 & 3 & -3 & 12 & 2 \\
\hline 158 & 3 & -4 & 13 & 3 \\
\hline 158 & 3 & -4 & 14 & 4 \\
\hline 158 & 3 & -4 & 15 & 5 \\
\hline 159 & 3 & -3 & 11 & 1 \\
\hline 159 & 3 & -4 & 12 & 2 \\
\hline 159 & 3 & -4 & 13 & 3 \\
\hline 159 & 3 & -4 & 14 & 4 \\
\hline 159 & 3 & -4 & 15 & 5 \\
\hline
\end{tabular}




\begin{tabular}{|c|c|c|c|c|}
\hline 160 & 3 & -2 & 11 & 1 \\
\hline 160 & 3 & -3 & 12 & 2 \\
\hline 160 & 3 & -3 & 13 & 3 \\
\hline 160 & 3 & -4 & 14 & 4 \\
\hline 160 & 3 & -4 & 15 & 5 \\
\hline 161 & 3 & 1 & 11 & 1 \\
\hline 161 & 3 & -1 & 12 & 2 \\
\hline 161 & 3 & -2 & 13 & 3 \\
\hline 161 & 3 & -3 & 14 & 4 \\
\hline 161 & 3 & -4 & 15 & 5 \\
\hline 162 & 3 & -1 & 11 & 1 \\
\hline 162 & 3 & -1 & 12 & 2 \\
\hline 162 & 3 & -1 & 13 & 3 \\
\hline 162 & 3 & -1 & 14 & 4 \\
\hline 162 & 3 & -2 & 15 & 5 \\
\hline 163 & 3 & -2 & 11 & 1 \\
\hline 163 & 3 & -2 & 12 & 2 \\
\hline 163 & 3 & -2 & 13 & 3 \\
\hline 163 & 3 & -2 & 14 & 4 \\
\hline 163 & 3 & -3 & 15 & 5 \\
\hline 164 & 3 & -1 & 11 & 1 \\
\hline 164 & 3 & -2 & 12 & 2 \\
\hline 164 & 3 & -2 & 13 & 3 \\
\hline 164 & 3 & -3 & 14 & 4 \\
\hline 164 & 3 & -3 & 15 & 5 \\
\hline 165 & 3 & 2 & 11 & 1 \\
\hline 165 & 3 & -2 & 12 & 2 \\
\hline 165 & 3 & -1 & 13 & 3 \\
\hline 165 & 3 & -3 & 14 & 4 \\
\hline 165 & 3 & 0 & 15 & 5 \\
\hline 166 & 3 & -2 & 11 & 1 \\
\hline 166 & 3 & -3 & 12 & 2 \\
\hline 166 & 3 & -3 & 13 & 3 \\
\hline 166 & 3 & -3 & 14 & 4 \\
\hline 166 & 3 & -3 & 15 & 5 \\
\hline 167 & 3 & 4 & 11 & 1 \\
\hline 167 & 3 & 4 & 12 & 2 \\
\hline 167 & 3 & 4 & 13 & 3 \\
\hline 167 & 3 & 4 & 14 & 4 \\
\hline 167 & 3 & 4 & 15 & 5 \\
\hline 168 & 3 & -2 & 11 & 1 \\
\hline 168 & 3 & -2 & 12 & 2 \\
\hline 168 & 3 & -2 & 13 & 3 \\
\hline 168 & 3 & -2 & 14 & 4 \\
\hline 168 & 3 & -3 & 15 & 5 \\
\hline 169 & 3 & -2 & 11 & 1 \\
\hline 169 & 3 & -2 & 12 & 2 \\
\hline 169 & 3 & -3 & 13 & 3 \\
\hline 169 & 3 & -3 & 14 & 4 \\
\hline 169 & 3 & -3 & 15 & 5 \\
\hline 170 & 3 & 2 & 11 & 1 \\
\hline
\end{tabular}




\begin{tabular}{|c|c|c|c|c|}
\hline 170 & 3 & 2 & 12 & 2 \\
\hline 170 & 3 & -1 & 13 & 3 \\
\hline 170 & 3 & -1 & 14 & 4 \\
\hline 170 & 3 & -1 & 15 & 5 \\
\hline 171 & 3 & 2 & 11 & 1 \\
\hline 171 & 3 & 2 & 12 & 2 \\
\hline 171 & 3 & 2 & 13 & 3 \\
\hline 171 & 3 & 2 & 14 & 4 \\
\hline 171 & 3 & 2 & 15 & 5 \\
\hline 172 & 3 & -3 & 11 & 1 \\
\hline 172 & 3 & -4 & 12 & 2 \\
\hline 172 & 3 & -4 & 13 & 3 \\
\hline 172 & 3 & -4 & 14 & 4 \\
\hline 172 & 3 & -4 & 15 & 5 \\
\hline 173 & 3 & -1 & 11 & 1 \\
\hline 173 & 3 & -2 & 12 & 2 \\
\hline 173 & 3 & -2 & 13 & 3 \\
\hline 173 & 3 & -2 & 14 & 4 \\
\hline 173 & 3 & -2 & 15 & 5 \\
\hline 174 & 4 & 4 & 1 & 1 \\
\hline 174 & 4 & 2 & 4 & 2 \\
\hline 174 & 4 & -2 & 7 & 3 \\
\hline 174 & 4 & -4 & 10 & 4 \\
\hline 174 & 4 & -4 & 13 & 5 \\
\hline 175 & 4 & 4 & 1 & 1 \\
\hline 175 & 4 & 3 & 4 & 2 \\
\hline 175 & 4 & 2 & 7 & 3 \\
\hline 175 & 4 & -1 & 10 & 4 \\
\hline 175 & 4 & -3 & 13 & 5 \\
\hline 176 & 4 & 4 & 1 & 1 \\
\hline 176 & 4 & -1 & 4 & 2 \\
\hline 176 & 4 & -3 & 7 & 3 \\
\hline 176 & 4 & -4 & 10 & 4 \\
\hline 176 & 4 & -4 & 13 & 5 \\
\hline 177 & 4 & 4 & 1 & 1 \\
\hline 177 & 4 & -1 & 4 & 2 \\
\hline 177 & 4 & -2 & 7 & 3 \\
\hline 177 & 4 & -4 & 10 & 4 \\
\hline 177 & 4 & -4 & 13 & 5 \\
\hline 178 & 4 & 4 & 1 & 1 \\
\hline 178 & 4 & 3 & 4 & 2 \\
\hline 178 & 4 & 2 & 7 & 3 \\
\hline 178 & 4 & 0 & 10 & 4 \\
\hline 178 & 4 & -2 & 13 & 5 \\
\hline 179 & 4 & 4 & 1 & 1 \\
\hline 179 & 4 & -3 & 4 & 2 \\
\hline 179 & 4 & -4 & 7 & 3 \\
\hline 179 & 4 & -4 & 10 & 4 \\
\hline 179 & 4 & -4 & 13 & 5 \\
\hline 180 & 4 & 4 & 1 & 1 \\
\hline 180 & 4 & 4 & 4 & 2 \\
\hline
\end{tabular}




\begin{tabular}{|c|c|c|c|c|}
\hline 180 & 4 & 4 & 7 & 3 \\
\hline 180 & 4 & 4 & 10 & 4 \\
\hline 180 & 4 & 4 & 13 & 5 \\
\hline 181 & 4 & 4 & 1 & 1 \\
\hline 181 & 4 & 4 & 4 & 2 \\
\hline 181 & 4 & 3 & 7 & 3 \\
\hline 181 & 4 & 3 & 10 & 4 \\
\hline 181 & 4 & 3 & 13 & 5 \\
\hline 182 & 4 & 4 & 1 & 1 \\
\hline 182 & 4 & -2 & 4 & 2 \\
\hline 182 & 4 & -2 & 7 & 3 \\
\hline 182 & 4 & -3 & 10 & 4 \\
\hline 182 & 4 & & 13 & 5 \\
\hline 183 & 4 & 4 & 1 & 1 \\
\hline 183 & 4 & 2 & 4 & 2 \\
\hline 183 & 4 & 0 & 7 & 3 \\
\hline 183 & 4 & -3 & 10 & 4 \\
\hline 183 & 4 & -4 & 13 & 5 \\
\hline 184 & 4 & 4 & 1 & 1 \\
\hline 184 & 4 & -2 & 4 & 2 \\
\hline 184 & 4 & -3 & 7 & 3 \\
\hline 184 & 4 & -3 & 10 & 4 \\
\hline 184 & 4 & -4 & 13 & 5 \\
\hline 185 & 4 & 4 & 1 & 1 \\
\hline 185 & 4 & 1 & 4 & 2 \\
\hline 185 & 4 & 0 & 7 & 3 \\
\hline 185 & 4 & -2 & 10 & 4 \\
\hline 185 & 4 & -2 & 13 & 5 \\
\hline 186 & 4 & 4 & 1 & 1 \\
\hline 186 & 4 & 1 & 4 & 2 \\
\hline 186 & 4 & -1 & 7 & 3 \\
\hline 186 & 4 & -3 & 10 & 4 \\
\hline 186 & 4 & -3 & 13 & 5 \\
\hline 187 & 4 & 4 & 1 & 1 \\
\hline 187 & 4 & -4 & 4 & 2 \\
\hline 187 & 4 & -4 & 7 & 3 \\
\hline 187 & 4 & -4 & 10 & 4 \\
\hline 187 & 4 & -4 & 13 & 5 \\
\hline 188 & 4 & 3 & 1 & 1 \\
\hline 188 & 4 & 2 & 4 & 2 \\
\hline 188 & 4 & 1 & 7 & 3 \\
\hline 188 & 4 & -1 & 10 & 4 \\
\hline 188 & 4 & -3 & 13 & 5 \\
\hline 189 & 4 & 4 & 1 & 1 \\
\hline 189 & 4 & 1 & 4 & 2 \\
\hline 189 & 4 & -3 & 7 & 3 \\
\hline 189 & 4 & -4 & 10 & 4 \\
\hline 189 & 4 & -4 & 13 & 5 \\
\hline 190 & 4 & 4 & 1 & 1 \\
\hline 190 & 4 & 3 & 4 & 2 \\
\hline 190 & 4 & 1 & 7 & 3 \\
\hline
\end{tabular}




\begin{tabular}{|c|c|c|c|c|}
\hline 190 & 4 & -2 & 10 & 4 \\
\hline 190 & 4 & -3 & 13 & 5 \\
\hline 191 & 4 & 4 & 1 & 1 \\
\hline 191 & 4 & 2 & 4 & 2 \\
\hline 191 & 4 & -1 & 7 & 3 \\
\hline 191 & 4 & -4 & 10 & 4 \\
\hline 191 & 4 & -4 & 13 & 5 \\
\hline 192 & 4 & 4 & 1 & 1 \\
\hline 192 & 4 & 1 & 4 & 2 \\
\hline 192 & 4 & -2 & 7 & 3 \\
\hline 192 & 4 & -3 & 10 & 4 \\
\hline 192 & 4 & -4 & 13 & 5 \\
\hline 193 & 4 & 4 & 1 & 1 \\
\hline 193 & 4 & 1 & 4 & 2 \\
\hline 193 & 4 & -2 & 7 & 3 \\
\hline 193 & 4 & -4 & 10 & 4 \\
\hline 193 & 4 & -4 & 13 & 5 \\
\hline 194 & 4 & 4 & 1 & 1 \\
\hline 194 & 4 & 3 & 4 & 2 \\
\hline 194 & 4 & 2 & 7 & 3 \\
\hline 194 & 4 & -2 & 10 & 4 \\
\hline 194 & 4 & -4 & 13 & 5 \\
\hline 195 & 4 & 4 & 1 & 1 \\
\hline 195 & 4 & 1 & 4 & 2 \\
\hline 195 & 4 & -1 & 7 & 3 \\
\hline 195 & 4 & -4 & 10 & 4 \\
\hline 195 & 4 & -4 & 13 & 5 \\
\hline 196 & 4 & 4 & 1 & 1 \\
\hline 196 & 4 & 2 & 4 & 2 \\
\hline 196 & 4 & -1 & 7 & 3 \\
\hline 196 & 4 & -1 & 10 & 4 \\
\hline 196 & 4 & -3 & 13 & 5 \\
\hline 197 & 4 & 4 & 1 & 1 \\
\hline 197 & 4 & -2 & 4 & 2 \\
\hline 197 & 4 & -3 & 7 & 3 \\
\hline 197 & 4 & -4 & 10 & 4 \\
\hline 197 & 4 & -4 & 13 & 5 \\
\hline 198 & 4 & 4 & 1 & 1 \\
\hline 198 & 4 & -2 & 4 & 2 \\
\hline 198 & 4 & -4 & 7 & 3 \\
\hline 198 & 4 & -4 & 10 & 4 \\
\hline 198 & 4 & -4 & 13 & 5 \\
\hline 199 & 4 & 4 & 1 & 1 \\
\hline 199 & 4 & -1 & 4 & 2 \\
\hline 199 & 4 & -2 & 7 & 3 \\
\hline 199 & 4 & -3 & 10 & 4 \\
\hline 199 & 4 & -4 & 13 & 5 \\
\hline 200 & 4 & 4 & 1 & 1 \\
\hline 200 & 4 & 3 & 4 & 2 \\
\hline 200 & 4 & 0 & 7 & 3 \\
\hline 200 & 4 & -1 & 10 & 4 \\
\hline
\end{tabular}




\begin{tabular}{|c|c|c|c|c|}
\hline 200 & 4 & -2 & 13 & 5 \\
\hline 201 & 4 & 4 & 1 & 1 \\
\hline 201 & 4 & 1 & 4 & 2 \\
\hline 201 & 4 & -1 & 7 & 3 \\
\hline 201 & 4 & -3 & 10 & 4 \\
\hline 201 & 4 & -4 & 13 & 5 \\
\hline 202 & 4 & 4 & 1 & 1 \\
\hline 202 & 4 & 2 & 4 & 2 \\
\hline 202 & 4 & 2 & 7 & 3 \\
\hline 202 & 4 & 1 & 10 & 4 \\
\hline 202 & 4 & 0 & 13 & 5 \\
\hline 203 & 4 & 4 & 1 & 1 \\
\hline 203 & 4 & 2 & 4 & 2 \\
\hline 203 & 4 & 1 & 7 & 3 \\
\hline 203 & 4 & -1 & 10 & 4 \\
\hline 203 & 4 & -3 & 13 & 5 \\
\hline 204 & 4 & 4 & 1 & 1 \\
\hline 204 & 4 & 1 & 4 & 2 \\
\hline 204 & 4 & -1 & 7 & 3 \\
\hline 204 & 4 & -3 & 10 & 4 \\
\hline 204 & 4 & -4 & 13 & 5 \\
\hline 205 & 4 & 4 & 1 & 1 \\
\hline 205 & 4 & 4 & 4 & 2 \\
\hline 205 & 4 & 2 & 7 & 3 \\
\hline 205 & 4 & -1 & 10 & 4 \\
\hline 205 & 4 & -1 & 13 & 5 \\
\hline 206 & 4 & 4 & 1 & 1 \\
\hline 206 & 4 & 1 & 4 & 2 \\
\hline 206 & 4 & -1 & 7 & 3 \\
\hline 206 & 4 & -3 & 10 & 4 \\
\hline 206 & 4 & -4 & 13 & 5 \\
\hline 207 & 4 & 4 & 1 & 1 \\
\hline 207 & 4 & 3 & 4 & 2 \\
\hline 207 & 4 & 0 & 7 & 3 \\
\hline 207 & 4 & -2 & 10 & 4 \\
\hline 207 & 4 & -3 & 13 & 5 \\
\hline 208 & 4 & 4 & 1 & 1 \\
\hline 208 & 4 & 3 & 4 & 2 \\
\hline 208 & 4 & 2 & 7 & 3 \\
\hline 208 & 4 & 0 & 10 & 4 \\
\hline 208 & 4 & 0 & 13 & 5 \\
\hline 209 & 4 & 4 & 1 & 1 \\
\hline 209 & 4 & 1 & 4 & 2 \\
\hline 209 & 4 & -1 & 7 & 3 \\
\hline 209 & 4 & -3 & 10 & 4 \\
\hline 209 & 4 & -4 & 13 & 5 \\
\hline 210 & 5 & 3 & 3 & 3 \\
\hline 210 & 5 & 1 & 6 & 1 \\
\hline 210 & 5 & -3 & 9 & -3 \\
\hline 210 & 5 & -4 & 12 & -4 \\
\hline 210 & 5 & -4 & 15 & 5 \\
\hline
\end{tabular}




\begin{tabular}{|c|c|c|c|c|}
\hline 211 & 5 & 3 & 3 & 1 \\
\hline 211 & 5 & -1 & 6 & 2 \\
\hline 211 & 5 & -3 & 9 & 3 \\
\hline 211 & 5 & -4 & 12 & 4 \\
\hline 211 & 5 & -4 & 15 & 5 \\
\hline 212 & 5 & 2 & 3 & 1 \\
\hline 212 & 5 & -3 & 6 & 2 \\
\hline 212 & 5 & -4 & 9 & 3 \\
\hline 212 & 5 & -4 & 12 & 4 \\
\hline 212 & 5 & -4 & 15 & 5 \\
\hline 213 & 5 & 4 & 3 & 1 \\
\hline 213 & 5 & 2 & 6 & 2 \\
\hline 213 & 5 & 0 & 9 & 3 \\
\hline 213 & 5 & -1 & 12 & 4 \\
\hline 213 & 5 & -2 & 15 & 5 \\
\hline 214 & 5 & 4 & 3 & 1 \\
\hline 214 & 5 & 3 & 6 & 2 \\
\hline 214 & 5 & 2 & 9 & 3 \\
\hline 214 & 5 & -1 & 12 & 4 \\
\hline 214 & 5 & -2 & 15 & 5 \\
\hline 215 & 5 & 2 & 3 & 1 \\
\hline 215 & 5 & -2 & 6 & 2 \\
\hline 215 & 5 & -4 & 9 & 3 \\
\hline 215 & 5 & -1 & 12 & 4 \\
\hline 215 & 5 & -2 & 15 & 5 \\
\hline 216 & 5 & 2 & 3 & 1 \\
\hline 216 & 5 & 0 & 6 & 2 \\
\hline 216 & 5 & -1 & 9 & 3 \\
\hline 216 & 5 & -4 & 12 & 4 \\
\hline 216 & 5 & -4 & 15 & 5 \\
\hline 217 & 5 & 1 & 3 & 1 \\
\hline 217 & 5 & -4 & 6 & 2 \\
\hline 217 & 5 & -4 & 9 & 3 \\
\hline 217 & 5 & -2 & 12 & 4 \\
\hline 217 & 5 & -2 & 15 & 5 \\
\hline 218 & 5 & 0 & 3 & 1 \\
\hline 218 & 5 & -2 & 6 & 2 \\
\hline 218 & 5 & -3 & 9 & 3 \\
\hline 218 & 5 & -4 & 12 & 4 \\
\hline 218 & 5 & -4 & 15 & 5 \\
\hline 219 & 5 & 3 & 3 & 1 \\
\hline 219 & 5 & 0 & 6 & 2 \\
\hline 219 & 5 & -2 & 9 & 3 \\
\hline 219 & 5 & -4 & 12 & 4 \\
\hline 219 & 5 & -4 & 15 & 5 \\
\hline 220 & 5 & 3 & 3 & 1 \\
\hline 220 & 5 & 1 & 6 & 2 \\
\hline 220 & 5 & -2 & 9 & 3 \\
\hline 220 & 5 & -3 & 12 & 4 \\
\hline 220 & 5 & -3 & 15 & 5 \\
\hline 221 & 5 & 4 & 3 & 1 \\
\hline
\end{tabular}




\begin{tabular}{|c|c|c|c|c|}
\hline 221 & 5 & 1 & 6 & 2 \\
\hline 221 & 5 & -1 & 9 & 3 \\
\hline 221 & 5 & -3 & 12 & 4 \\
\hline 221 & 5 & -4 & 15 & 5 \\
\hline 222 & 5 & 3 & 3 & 1 \\
\hline 222 & 5 & 1 & 6 & 2 \\
\hline 222 & 5 & -1 & 9 & 3 \\
\hline 222 & 5 & -2 & 12 & 4 \\
\hline 222 & 5 & -3 & 15 & 5 \\
\hline 223 & 5 & 4 & 3 & 1 \\
\hline 223 & 5 & 4 & 6 & 2 \\
\hline 223 & 5 & 3 & 9 & 3 \\
\hline 223 & 5 & -3 & 12 & 4 \\
\hline 223 & 5 & -4 & 15 & 5 \\
\hline 224 & 5 & 2 & 3 & 1 \\
\hline 224 & 5 & 2 & 6 & 2 \\
\hline 224 & 5 & 0 & 9 & 3 \\
\hline 224 & 5 & -2 & 12 & 4 \\
\hline 224 & 5 & -3 & 15 & 5 \\
\hline 225 & 5 & 2 & 3 & 1 \\
\hline 225 & 5 & 1 & 6 & 2 \\
\hline 225 & 5 & 1 & 9 & 3 \\
\hline 225 & 5 & 2 & 12 & 4 \\
\hline 225 & 5 & 2 & 15 & 5 \\
\hline 226 & 5 & 2 & 3 & 1 \\
\hline 226 & 5 & -1 & 6 & 2 \\
\hline 226 & 5 & -1 & 9 & 3 \\
\hline 226 & 5 & -1 & 12 & 4 \\
\hline 226 & 5 & -1 & 15 & 5 \\
\hline 227 & 5 & 2 & 3 & 1 \\
\hline 227 & 5 & 0 & 6 & 2 \\
\hline 227 & 5 & -2 & 9 & 3 \\
\hline 227 & 5 & 0 & 12 & 4 \\
\hline 227 & 5 & 0 & 15 & 5 \\
\hline 228 & 5 & -1 & 3 & 1 \\
\hline 228 & 5 & -3 & 6 & 2 \\
\hline 228 & 5 & -4 & 9 & 3 \\
\hline 228 & 5 & -3 & 12 & 4 \\
\hline 228 & 5 & -4 & 15 & 5 \\
\hline 229 & 5 & 2 & 3 & 1 \\
\hline 229 & 5 & 0 & 6 & 2 \\
\hline 229 & 5 & -1 & 9 & 3 \\
\hline 229 & 5 & -2 & 12 & 4 \\
\hline 229 & 5 & -3 & 15 & 5 \\
\hline 230 & 5 & 2 & 3 & 1 \\
\hline 230 & 5 & 1 & 6 & 2 \\
\hline 230 & 5 & -1 & 9 & 3 \\
\hline 230 & 5 & -4 & 12 & 4 \\
\hline 230 & 5 & -4 & 15 & 5 \\
\hline 231 & 5 & 3 & 3 & 1 \\
\hline 231 & 5 & 1 & 6 & 2 \\
\hline
\end{tabular}




\begin{tabular}{|c|c|c|c|c|}
\hline 231 & 5 & -1 & 9 & 3 \\
\hline 231 & 5 & -1 & 12 & 4 \\
\hline 231 & 5 & -3 & 15 & 5 \\
\hline 232 & 5 & 4 & 3 & 1 \\
\hline 232 & 5 & 2 & 6 & 2 \\
\hline 232 & 5 & 0 & 9 & 3 \\
\hline 232 & 5 & -2 & 12 & 4 \\
\hline 232 & 5 & -3 & 15 & 5 \\
\hline 233 & 5 & 4 & 3 & 1 \\
\hline 233 & 5 & 4 & 6 & 2 \\
\hline 233 & 5 & 1 & 9 & 3 \\
\hline 233 & 5 & -3 & 12 & 4 \\
\hline 233 & 5 & -4 & 15 & 5 \\
\hline 234 & 5 & 1 & 3 & 1 \\
\hline 234 & 5 & -1 & 6 & 2 \\
\hline 234 & 5 & -3 & 9 & 3 \\
\hline 234 & 5 & -3 & 12 & 4 \\
\hline 234 & 5 & -4 & 15 & 5 \\
\hline 235 & 5 & 4 & 3 & 1 \\
\hline 235 & 5 & 2 & 6 & 2 \\
\hline 235 & 5 & -1 & 9 & 3 \\
\hline 235 & 5 & -1 & 12 & 4 \\
\hline 235 & 5 & -2 & 15 & 5 \\
\hline 236 & 5 & 4 & 3 & 1 \\
\hline 236 & 5 & 3 & 6 & 2 \\
\hline 236 & 5 & 3 & 9 & 3 \\
\hline 236 & 5 & -4 & 12 & 4 \\
\hline 236 & 5 & -4 & 15 & 5 \\
\hline 237 & 5 & 3 & 3 & 1 \\
\hline 237 & 5 & 2 & 6 & 2 \\
\hline 237 & 5 & 1 & 9 & 3 \\
\hline 237 & 5 & -2 & 12 & 4 \\
\hline 237 & 5 & -4 & 15 & 5 \\
\hline 238 & 5 & 3 & 3 & 1 \\
\hline 238 & 5 & 1 & 6 & 2 \\
\hline 238 & 5 & -1 & 9 & 3 \\
\hline 238 & 5 & 2 & 12 & 4 \\
\hline 238 & 5 & 0 & 15 & 5 \\
\hline 239 & 5 & 4 & 3 & 1 \\
\hline 239 & 5 & 2 & 6 & 2 \\
\hline 239 & 5 & -1 & 9 & 3 \\
\hline 239 & 5 & 0 & 12 & 4 \\
\hline 239 & 5 & -3 & 15 & 5 \\
\hline 240 & 5 & 2 & 3 & 1 \\
\hline 240 & 5 & 0 & 6 & 2 \\
\hline 240 & 5 & -2 & 9 & 3 \\
\hline 240 & 5 & -3 & 12 & 4 \\
\hline 240 & 5 & -4 & 15 & 5 \\
\hline 241 & 5 & 4 & 3 & 1 \\
\hline 241 & 5 & 1 & 6 & 2 \\
\hline 241 & 5 & -2 & 9 & 3 \\
\hline
\end{tabular}




\begin{tabular}{|c|c|c|c|c|}
\hline 241 & 5 & -2 & 12 & 4 \\
\hline 241 & 5 & -4 & 15 & 5 \\
\hline 242 & 5 & -2 & 3 & 1 \\
\hline 242 & 5 & -3 & 6 & 2 \\
\hline 242 & 5 & -4 & 9 & 3 \\
\hline 242 & 5 & -2 & 12 & 4 \\
\hline 242 & 5 & -3 & 15 & 5 \\
\hline 243 & 5 & 4 & 3 & 1 \\
\hline 243 & 5 & 2 & 6 & 2 \\
\hline 243 & 5 & 1 & 9 & 3 \\
\hline 243 & 5 & -4 & 12 & 4 \\
\hline 243 & 5 & -4 & 15 & 5 \\
\hline 244 & 5 & 3 & 3 & 1 \\
\hline 244 & 5 & 1 & 6 & 2 \\
\hline 244 & 5 & 0 & 9 & 3 \\
\hline 244 & 5 & -2 & 12 & 4 \\
\hline 244 & 5 & -3 & 15 & 5 \\
\hline 245 & 6 & 4 & 1 & 1 \\
\hline 245 & 6 & -1 & 3 & 2 \\
\hline 245 & 6 & -1 & 5 & 3 \\
\hline 245 & 6 & -4 & 7 & 4 \\
\hline 245 & 6 & -4 & 9 & 5 \\
\hline 246 & 6 & 4 & 1 & 1 \\
\hline 246 & 6 & 4 & 3 & 2 \\
\hline 246 & 6 & 4 & 5 & 3 \\
\hline 246 & 6 & -2 & 7 & 4 \\
\hline 246 & 6 & -3 & 9 & 5 \\
\hline 247 & 6 & 4 & 1 & 1 \\
\hline 247 & 6 & 2 & 3 & 2 \\
\hline 247 & 6 & 2 & 5 & 3 \\
\hline 247 & 6 & -2 & 7 & 4 \\
\hline 247 & 6 & -4 & 9 & 5 \\
\hline 248 & 6 & 4 & 1 & 1 \\
\hline 248 & 6 & 3 & 3 & 2 \\
\hline 248 & 6 & 3 & 5 & 3 \\
\hline 248 & 6 & -4 & 7 & 4 \\
\hline 248 & 6 & -4 & 9 & 5 \\
\hline 249 & 6 & 4 & 1 & 1 \\
\hline 249 & 6 & 3 & 3 & 2 \\
\hline 249 & 6 & 3 & 5 & 3 \\
\hline 249 & 6 & -3 & 7 & 4 \\
\hline 249 & 6 & -4 & 9 & 5 \\
\hline 250 & 6 & 4 & 1 & 1 \\
\hline 250 & 6 & 2 & 3 & 2 \\
\hline 250 & 6 & 2 & 5 & 3 \\
\hline 250 & 6 & -2 & 7 & 4 \\
\hline 250 & 6 & -3 & 9 & 5 \\
\hline 251 & 6 & 4 & 1 & 1 \\
\hline 251 & 6 & -1 & 3 & 2 \\
\hline 251 & 6 & -1 & 5 & 3 \\
\hline 251 & 6 & -4 & 7 & 4 \\
\hline
\end{tabular}




\begin{tabular}{|c|c|c|c|c|}
\hline 251 & 6 & -4 & 9 & 5 \\
\hline 252 & 6 & 4 & 1 & 1 \\
\hline 252 & 6 & 3 & 3 & 2 \\
\hline 252 & 6 & 3 & 5 & 3 \\
\hline 252 & 6 & -2 & 7 & 4 \\
\hline 252 & 6 & -4 & 9 & 5 \\
\hline 253 & 6 & 4 & 1 & 1 \\
\hline 253 & 6 & 2 & 3 & 2 \\
\hline 253 & 6 & 2 & 5 & 3 \\
\hline 253 & 6 & -2 & 7 & 4 \\
\hline 253 & 6 & -4 & 9 & 5 \\
\hline 254 & 6 & 4 & 1 & 1 \\
\hline 254 & 6 & 3 & 3 & 2 \\
\hline 254 & 6 & 3 & 5 & 3 \\
\hline 254 & 6 & 1 & 7 & 4 \\
\hline 254 & 6 & 0 & 9 & 5 \\
\hline 255 & 6 & 4 & 1 & 1 \\
\hline 255 & 6 & 3 & 3 & 2 \\
\hline 255 & 6 & 3 & 5 & 3 \\
\hline 255 & 6 & -1 & 7 & 4 \\
\hline 255 & 6 & -3 & 9 & 5 \\
\hline 256 & 6 & 4 & 1 & 1 \\
\hline 256 & 6 & 3 & 3 & 2 \\
\hline 256 & 6 & 3 & 5 & 3 \\
\hline 256 & 6 & -2 & 7 & 4 \\
\hline 256 & 6 & -4 & 9 & 5 \\
\hline 257 & 6 & 4 & 1 & 1 \\
\hline 257 & 6 & 3 & 3 & 2 \\
\hline 257 & 6 & 3 & 5 & 3 \\
\hline 257 & 6 & -1 & 7 & 4 \\
\hline 257 & 6 & -2 & 9 & 5 \\
\hline 258 & 6 & 4 & 1 & 1 \\
\hline 258 & 6 & 3 & 3 & 2 \\
\hline 258 & 6 & 3 & 5 & 3 \\
\hline 258 & 6 & 2 & 7 & 4 \\
\hline 258 & 6 & -2 & 9 & 5 \\
\hline 259 & 6 & 4 & 1 & 1 \\
\hline 259 & 6 & 4 & 3 & 2 \\
\hline 259 & 6 & 4 & 5 & 3 \\
\hline 259 & 6 & -1 & 7 & 4 \\
\hline 259 & 6 & -3 & 9 & 5 \\
\hline 260 & 6 & 4 & 1 & 1 \\
\hline 260 & 6 & 4 & 3 & 2 \\
\hline 260 & 6 & 4 & 5 & 3 \\
\hline 260 & 6 & -1 & 7 & 4 \\
\hline 260 & 6 & -3 & 9 & 5 \\
\hline 261 & 6 & 4 & 1 & 1 \\
\hline 261 & 6 & 3 & 3 & 2 \\
\hline 261 & 6 & 3 & 5 & 3 \\
\hline 261 & 6 & 1 & 7 & 4 \\
\hline 261 & 6 & 1 & 9 & 5 \\
\hline
\end{tabular}




\begin{tabular}{|c|c|c|c|c|}
\hline 262 & 6 & 4 & 1 & 1 \\
\hline 262 & 6 & 4 & 3 & 2 \\
\hline 262 & 6 & 4 & 5 & 3 \\
\hline 262 & 6 & 0 & 7 & 4 \\
\hline 262 & 6 & -2 & 9 & 5 \\
\hline 263 & 6 & -1 & 1 & 1 \\
\hline 263 & 6 & 1 & 3 & 2 \\
\hline 263 & 6 & 1 & 5 & 3 \\
\hline 263 & 6 & -2 & 7 & 4 \\
\hline 263 & 6 & -2 & 9 & 5 \\
\hline 264 & 6 & 4 & 1 & 1 \\
\hline 264 & 6 & 4 & 3 & 2 \\
\hline 264 & 6 & 4 & 5 & 3 \\
\hline 264 & 6 & 1 & 7 & 4 \\
\hline 264 & 6 & -1 & 9 & 5 \\
\hline 265 & 6 & 4 & 1 & 1 \\
\hline 265 & 6 & 4 & 3 & 2 \\
\hline 265 & 6 & 4 & 5 & 3 \\
\hline 265 & 6 & -1 & 7 & 4 \\
\hline 265 & 6 & -3 & 9 & 5 \\
\hline 266 & 6 & 4 & 1 & 1 \\
\hline 266 & 6 & 4 & 3 & 2 \\
\hline 266 & 6 & 4 & 5 & 3 \\
\hline 266 & 6 & 1 & 7 & 4 \\
\hline 266 & 6 & -1 & 9 & 5 \\
\hline 267 & 6 & 4 & 1 & 1 \\
\hline 267 & 6 & 2 & 3 & 2 \\
\hline 267 & 6 & 2 & 5 & 3 \\
\hline 267 & 6 & -2 & 7 & 4 \\
\hline 267 & 6 & -4 & 9 & 5 \\
\hline 268 & 6 & 3 & 1 & 1 \\
\hline 268 & 6 & 2 & 3 & 2 \\
\hline 268 & 6 & 2 & 5 & 3 \\
\hline 268 & 6 & -4 & 7 & 4 \\
\hline 268 & 6 & -4 & 9 & 5 \\
\hline 269 & 6 & 4 & 1 & 1 \\
\hline 269 & 6 & -1 & 3 & 2 \\
\hline 269 & 6 & -1 & 5 & 3 \\
\hline 269 & 6 & -4 & 7 & 4 \\
\hline 269 & 6 & -4 & 9 & 5 \\
\hline 270 & 6 & 4 & 1 & 1 \\
\hline 270 & 6 & 4 & 3 & 2 \\
\hline 270 & 6 & 4 & 5 & 3 \\
\hline 270 & 6 & 3 & 7 & 4 \\
\hline 270 & 6 & 3 & 9 & 5 \\
\hline 271 & 6 & 4 & 1 & 1 \\
\hline 271 & 6 & 4 & 3 & 2 \\
\hline 271 & 6 & 4 & 5 & 3 \\
\hline 271 & 6 & 1 & 7 & 4 \\
\hline 271 & 6 & -1 & 9 & 5 \\
\hline 272 & 6 & 4 & 1 & 1 \\
\hline
\end{tabular}




\begin{tabular}{|c|c|c|c|c|}
\hline 272 & 6 & 0 & 3 & 2 \\
\hline 272 & 6 & 0 & 5 & 3 \\
\hline 272 & 6 & -4 & 7 & 4 \\
\hline 272 & 6 & -4 & 9 & 5 \\
\hline 273 & 6 & 4 & 1 & 1 \\
\hline 273 & 6 & 3 & 3 & 2 \\
\hline 273 & 6 & 3 & 5 & 3 \\
\hline 273 & 6 & 0 & 7 & 4 \\
\hline 273 & 6 & -3 & 9 & 5 \\
\hline 274 & 6 & 4 & 1 & 1 \\
\hline 274 & 6 & 1 & 3 & 2 \\
\hline 274 & 6 & 1 & 5 & 3 \\
\hline 274 & 6 & -1 & 7 & 4 \\
\hline 274 & 6 & -1 & 9 & 5 \\
\hline 275 & 6 & 4 & 1 & 1 \\
\hline 275 & 6 & 2 & 3 & 2 \\
\hline 275 & 6 & 2 & 5 & 3 \\
\hline 275 & 6 & -1 & 7 & 4 \\
\hline 275 & 6 & -3 & 9 & 5 \\
\hline 276 & 6 & 4 & 1 & 1 \\
\hline 276 & 6 & 3 & 3 & 2 \\
\hline 276 & 6 & 3 & 5 & 3 \\
\hline 276 & 6 & -2 & 7 & 4 \\
\hline 276 & 6 & -2 & 9 & 5 \\
\hline 277 & 6 & 4 & 1 & 1 \\
\hline 277 & 6 & 2 & 3 & 2 \\
\hline 277 & 6 & 2 & 5 & 3 \\
\hline 277 & 6 & -4 & 7 & 4 \\
\hline 277 & 6 & -4 & 9 & 5 \\
\hline 278 & 7 & 2 & 7 & 1 \\
\hline 278 & 7 & 1 & 9 & 2 \\
\hline 278 & 7 & -1 & 11 & 3 \\
\hline 278 & 7 & -2 & 13 & 4 \\
\hline 278 & 7 & -3 & 15 & 5 \\
\hline 279 & 7 & -1 & 7 & 1 \\
\hline 279 & 7 & -1 & 9 & 2 \\
\hline 279 & 7 & -2 & 11 & 3 \\
\hline 279 & 7 & -2 & 13 & 4 \\
\hline 279 & 7 & -3 & 15 & 5 \\
\hline 280 & 7 & 1 & 7 & 1 \\
\hline 280 & 7 & 0 & 9 & 2 \\
\hline 280 & 7 & -1 & 11 & 3 \\
\hline 280 & 7 & -3 & 13 & 4 \\
\hline 280 & 7 & -4 & 15 & 5 \\
\hline 281 & 7 & 2 & 7 & 1 \\
\hline 281 & 7 & 2 & 9 & 2 \\
\hline 281 & 7 & 0 & 11 & 3 \\
\hline 281 & 7 & -1 & 13 & 4 \\
\hline 281 & 7 & -2 & 15 & 5 \\
\hline 282 & 7 & -3 & 7 & 1 \\
\hline 282 & 7 & -3 & 9 & 2 \\
\hline
\end{tabular}




\begin{tabular}{|c|c|c|c|c|}
\hline 282 & 7 & -3 & 11 & 3 \\
\hline 282 & 7 & -4 & 13 & 4 \\
\hline 282 & 7 & -4 & 15 & 5 \\
\hline 283 & 7 & 1 & 7 & 1 \\
\hline 283 & 7 & 1 & 9 & 2 \\
\hline 283 & 7 & 0 & 11 & 3 \\
\hline 283 & 7 & -2 & 13 & 4 \\
\hline 283 & 7 & -3 & 15 & 5 \\
\hline 284 & 7 & -3 & 7 & 1 \\
\hline 284 & 7 & -4 & 9 & 2 \\
\hline 284 & 7 & -4 & 11 & 3 \\
\hline 284 & 7 & -4 & 13 & 4 \\
\hline 284 & 7 & -4 & 15 & 5 \\
\hline 285 & 7 & 2 & 7 & 1 \\
\hline 285 & 7 & 0 & 9 & 2 \\
\hline 285 & 7 & -1 & 11 & 3 \\
\hline 285 & 7 & -2 & 13 & 4 \\
\hline 285 & 7 & -3 & 15 & 5 \\
\hline 286 & 7 & -4 & 7 & 1 \\
\hline 286 & 7 & -4 & 9 & 2 \\
\hline 286 & 7 & -4 & 11 & 3 \\
\hline 286 & 7 & -4 & 13 & 4 \\
\hline 286 & 7 & -4 & 15 & 5 \\
\hline 287 & 7 & 2 & 7 & 1 \\
\hline 287 & 7 & 1 & 9 & 2 \\
\hline 287 & 7 & 0 & 11 & 3 \\
\hline 287 & 7 & -2 & 13 & 4 \\
\hline 287 & 7 & -2 & 15 & 5 \\
\hline 288 & 7 & -3 & 7 & 1 \\
\hline 288 & 7 & -4 & 9 & 2 \\
\hline 288 & 7 & -4 & 11 & 3 \\
\hline 288 & 7 & -4 & 13 & 4 \\
\hline 288 & 7 & -4 & 15 & 5 \\
\hline 289 & 7 & 3 & 7 & 1 \\
\hline 289 & 7 & 2 & 9 & 2 \\
\hline 289 & 7 & 1 & 11 & 3 \\
\hline 289 & 7 & -2 & 13 & 4 \\
\hline 289 & 7 & -3 & 15 & 5 \\
\hline 290 & 7 & -1 & 7 & 1 \\
\hline 290 & 7 & -2 & 9 & 2 \\
\hline 290 & 7 & -2 & 11 & 3 \\
\hline 290 & 7 & -3 & 13 & 4 \\
\hline 290 & 7 & -4 & 15 & 5 \\
\hline 291 & 7 & -1 & 7 & 1 \\
\hline 291 & 7 & -2 & 9 & 2 \\
\hline 291 & 7 & -3 & 11 & 3 \\
\hline 291 & 7 & -4 & 13 & 4 \\
\hline 291 & 7 & -4 & 15 & 5 \\
\hline 292 & 7 & 3 & 7 & 1 \\
\hline 292 & 7 & 2 & 9 & 2 \\
\hline 292 & 7 & 1 & 11 & 3 \\
\hline
\end{tabular}




\begin{tabular}{|c|c|c|c|c|}
\hline 292 & 7 & -1 & 13 & 4 \\
\hline 292 & 7 & -2 & 15 & 5 \\
\hline 293 & 7 & 2 & 7 & 1 \\
\hline 293 & 7 & 2 & 9 & 2 \\
\hline 293 & 7 & 2 & 11 & 3 \\
\hline 293 & 7 & 1 & 13 & 4 \\
\hline 293 & 7 & 0 & 15 & 5 \\
\hline 294 & 7 & 4 & 7 & 1 \\
\hline 294 & 7 & 3 & 9 & 2 \\
\hline 294 & 7 & 2 & 11 & 3 \\
\hline 294 & 7 & 1 & 13 & 4 \\
\hline 294 & 7 & 0 & 15 & 5 \\
\hline 295 & 7 & -2 & 7 & 1 \\
\hline 295 & 7 & -2 & 9 & 2 \\
\hline 295 & 7 & -3 & 11 & 3 \\
\hline 295 & 7 & -3 & 13 & 4 \\
\hline 295 & 7 & -4 & 15 & 5 \\
\hline 296 & 7 & 2 & 7 & 1 \\
\hline 296 & 7 & 1 & 9 & 2 \\
\hline 296 & 7 & 1 & 11 & 3 \\
\hline 296 & 7 & 0 & 13 & 4 \\
\hline 296 & 7 & -1 & 15 & 5 \\
\hline 297 & 7 & -1 & 7 & 1 \\
\hline 297 & 7 & -2 & 9 & 2 \\
\hline 297 & 7 & -2 & 11 & 3 \\
\hline 297 & 7 & -2 & 13 & 4 \\
\hline 297 & 7 & -3 & 15 & 5 \\
\hline 298 & 7 & -4 & 7 & 1 \\
\hline 298 & 7 & -4 & 9 & 2 \\
\hline 298 & 7 & -4 & 11 & 3 \\
\hline 298 & 7 & -4 & 13 & 4 \\
\hline 298 & 7 & -4 & 15 & 5 \\
\hline 299 & 7 & 2 & 7 & 1 \\
\hline 299 & 7 & 1 & 9 & 2 \\
\hline 299 & 7 & -1 & 11 & 3 \\
\hline 299 & 7 & -3 & 13 & 4 \\
\hline 299 & 7 & -4 & 15 & 5 \\
\hline 300 & 7 & 0 & 7 & 1 \\
\hline 300 & 7 & -1 & 9 & 2 \\
\hline 300 & 7 & -1 & 11 & 3 \\
\hline 300 & 7 & -2 & 13 & 4 \\
\hline 300 & 7 & -3 & 15 & 5 \\
\hline 301 & 7 & 2 & 7 & 1 \\
\hline 301 & 7 & 1 & 9 & 2 \\
\hline 301 & 7 & -1 & 11 & 3 \\
\hline 301 & 7 & -2 & 13 & 4 \\
\hline 301 & 7 & -3 & 15 & 5 \\
\hline 302 & 7 & -3 & 7 & 1 \\
\hline 302 & 7 & -4 & 9 & 2 \\
\hline 302 & 7 & -4 & 11 & 3 \\
\hline 302 & 7 & -4 & 13 & 4 \\
\hline
\end{tabular}




\begin{tabular}{|c|c|c|c|c|}
\hline 302 & 7 & -4 & 15 & 5 \\
\hline 303 & 7 & 1 & 7 & 1 \\
\hline 303 & 7 & 0 & 9 & 2 \\
\hline 303 & 7 & -2 & 11 & 3 \\
\hline 303 & 7 & -3 & 13 & 4 \\
\hline 303 & 7 & -3 & 15 & 5 \\
\hline 304 & 7 & -1 & 7 & 1 \\
\hline 304 & 7 & -2 & 9 & 2 \\
\hline 304 & 7 & -2 & 11 & 3 \\
\hline 304 & 7 & -3 & 13 & 4 \\
\hline 304 & 7 & -3 & 15 & 5 \\
\hline 305 & 7 & -3 & 7 & 1 \\
\hline 305 & 7 & -4 & 9 & 2 \\
\hline 305 & 7 & -4 & 11 & 3 \\
\hline 305 & 7 & -4 & 13 & 4 \\
\hline 305 & 7 & -4 & 15 & 5 \\
\hline 306 & 7 & -1 & 7 & 1 \\
\hline 306 & 7 & 1 & 9 & 2 \\
\hline 306 & 7 & 2 & 11 & 3 \\
\hline 306 & 7 & 3 & 13 & 4 \\
\hline 306 & 7 & 4 & 15 & 5 \\
\hline 307 & 7 & 2 & 7 & 1 \\
\hline 307 & 7 & 1 & 9 & 2 \\
\hline 307 & 7 & 1 & 11 & 3 \\
\hline 307 & 7 & -1 & 13 & 4 \\
\hline 307 & 7 & -3 & 15 & 5 \\
\hline 308 & 7 & 4 & 7 & 1 \\
\hline 308 & 7 & 3 & 9 & 2 \\
\hline 308 & 7 & 3 & 11 & 3 \\
\hline 308 & 7 & 1 & 13 & 4 \\
\hline 308 & 7 & 1 & 15 & 5 \\
\hline 309 & 7 & -2 & 7 & 1 \\
\hline 309 & 7 & -2 & 9 & 2 \\
\hline 309 & 7 & -4 & 11 & 3 \\
\hline 309 & 7 & -4 & 13 & 4 \\
\hline 309 & 7 & -4 & 15 & 5 \\
\hline
\end{tabular}




\section{Appendix D: Range Effect R Script}

phNumber $<-$ seq (from $=0$, to $=14 * 3$, by $=3$ )

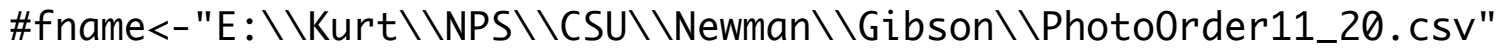

\#pRange<-read.table(fname, sep=', ', header=T) \# note that sep may

be ' $\backslash t$ ' for tab delimited

fname $<-F i l e$ location

pRange $<-\operatorname{scan}\left(\right.$ fname, what=double $(\theta), \operatorname{sep}={ }^{\prime},{ }^{\prime}$, skip=1) \# note that sep may be ' $\backslash t$ ' for tab delimited

pRange <- data.frame(t(array(pRange, dim=c(5, length(pRange)/5))) )

pRange <- pRange [,-5]

names(pRange) <- c("id", "grp", "accp", "phot")

pRange <- pRange[pRange\$accp >-8,]

pRange\$paot <- phNumber [pRange\$phot]

pRange $\$$ segq $<-0$

junk <- sapply(split(pRange\$phot,pRange\$grp), unique)

for (irow in seq(along=pRange $[, 1]$ ))

pRange\$seqq[irow] <-

match (pRange\$phot [irow], junk[,pRange\$grp[irow]])

nix <- pRange \$paot>0

modela <- $\operatorname{lm}($ accp $\sim$ paot, data=pRange)

modelb <- $\operatorname{lm}($ accp $\sim \log 10$ (paot), data=pRange [nix,])

modelc <- gam (accp s(paot), data=pRange)

modeld <- gam(accp s(paot), data=pRange[nix,])

modele <- $\operatorname{lm}($ accp $\sim$ paot, data=pRange $[$ nix,$])$

\# hairball plot

windows (hei $=8.5$, wi $d=11)$

$\operatorname{par}(\operatorname{mgp}=c(2,0.7,0), \operatorname{mar}=c(3.5,3.5,2,2))$

$\operatorname{plot}(1,1, x \lim =\mathrm{c}(0,42), y \lim =\mathrm{c}(-$

$4,4)$, type = 'n' , xlab="PAOT",ylab="Acceptability", axes=F,

frame $=$ T, cex. lab $=1.5$ )

$\operatorname{axis}(\operatorname{side}=1, a t=\operatorname{seq}($ from $=0, b y=3$, to $=42), l a b=\operatorname{seq}($ from $=0, b y=3$, to $=42)$ )

$\operatorname{axis}(\mathrm{side}=2, a \mathrm{t}=-4: 4, \mathrm{lab}=-4: 4)$

junk <- split(apply(pRange[,c(5,3)],2, jitter), pRange\$id)

junk <- sapply (junk, matrix, ncol=2)

sapply(junk, lines) 


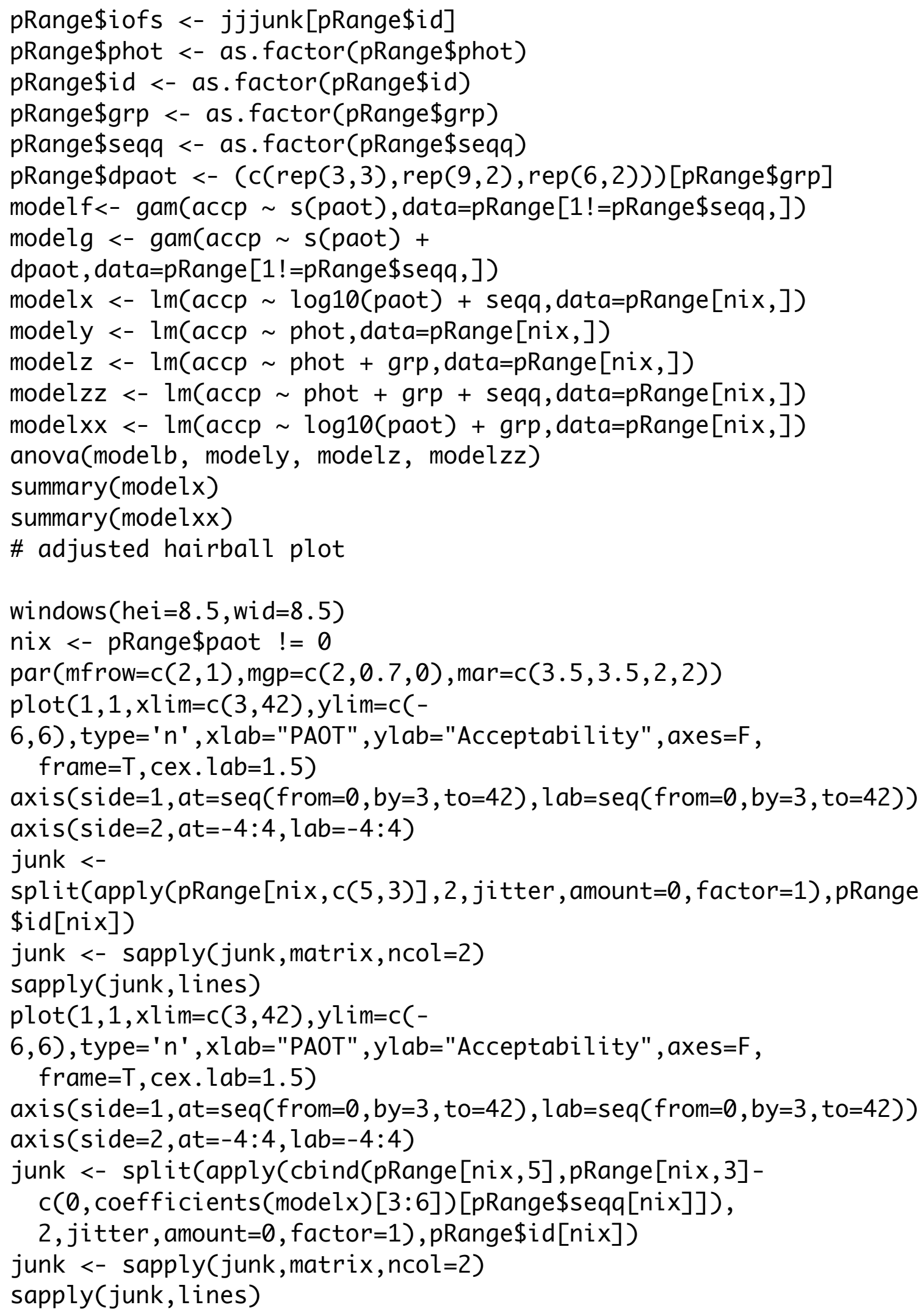


windows (he $i=8.5$, wid $=8.5$ )

$\operatorname{par}(\operatorname{mgp}=c(2,0.7,0), \operatorname{mar}=c(3.5,3.5,2,2))$

plot (jitter(pRange\$paot $[n i x])$, jitter(pRange\$accp $[n i x])$,

$x l a b=" P A 0 T ", y l a b=" A c c e p t a b i l i t y ", p c h=a s . n u m e r i c(p R a n g e \$ s e q q[n i x])$

,

axes $=\mathrm{F}$, frame. plot $=\mathrm{T}, \mathrm{cex} . \mathrm{lab}=1.5)$

$\operatorname{axis}($ side $=1, a t=\operatorname{seq}($ from $=3$, by $=3$, to $=42)$ )

axis ( $\operatorname{side}=2$, at $=$ seq $($ from $=-4$, to $=4)$ )

lines (unique(pRange\$paot) [-1], coefficients (modelb)[1]+

coefficients (modelb) [2]* $\log 10$ (unique(pRange $\$$ paot) [-1]), lwd=3)

junk <-

unique(cbind(pRange\$paot [nix], pRange\$seqq[nix], predict(modelx))

junk <- lapply(split(junk[,-2], junk[,2]), matrix, nco=2)

for (ic in junk $[c(1,5)]$ ) \{

ix $<-\operatorname{order}(i c[, 1])$

lines(ic $[i x, 1], i c[i x, 2]$, lwd $=2$ )

\}

legend $(x=39, y=3.75$, leg $=1: 5, p c h=1: 5)$

$\operatorname{text}(\mathrm{x}=15.5, \mathrm{y}=0.5, \mathrm{lab}=\operatorname{expression}(\mathrm{bolditalic}(" 1 \mathrm{st}$ photo")), pos=4) $\operatorname{text}(\mathrm{x}=20, \mathrm{y}=-1.5, \mathrm{lab}=\operatorname{expression}(\mathrm{bolditalic}(" 5$ th photo")), pos=4) if( 0$)\{$ \# this determined that the best fit for grp 7 was shifting $-0.5$

jjunk <-

apply (sapply (split(as. factor(pRange\$iofs), pRange\$grp), summary)[, c $(2,3,5)], 1$, sum $)$

jjjunk <-

sapply (split(as. factor (pRange \$iofs), pRange\$grp), summary) [,7]

jjunk <- jjunk $[-c(1: 2,4,6,8,10,12,14,16,18,20)]$

jjunk $<-$ jjunk/sum(jjunk)

jjjunk <- jjjunk $[-c(1: 3,5,7,9,11,13,15,17,19)]$

jjunk <- jjjunk/sum(jjjunk)

dist(rbind(jjunk, jjjunk))

jjjunk <- $c(\theta, j$ jjunk [-length(jjjunk $)])$

dist(rbind(jjunk, jjjunk))

\}

nix $<-1$ != pRange\$seqq

modelh <- gam(accp s(paot), data=pRange[nix, ])

modeli $<-\operatorname{gam}($ accp $\sim \mathrm{s}$ (paot) $+\mathrm{s}$ (iofs), data=pRange[nix,])

model j <- gam(accp s(paot) + s(iofs) + dpaot, data=pRange[nix,]) anova(modelh, model $i$, model $j$ )

$\operatorname{par}(\mathrm{mfrow}=\mathrm{c}(3,1), \mathrm{mgp}=\mathrm{c}(2, .7,0), \operatorname{mar}=\mathrm{c}(3,3,2.5,1), \mathrm{oma}=\mathrm{c}(2,1,2,1))$ plot $(j$ itter $(p$ Range $\$ a c c p[1 !=p R a n g e \$ s e q q])$, fitted (modelh), yl im=c($5,5)$, 
xlab="Acceptability",ylab="Predicted Acceptability")

title(expression("Acceptability" ==

bolditalic(f)(PAOT)), cex. main=2)

abline $(0,1)$

plot (jitter (pRange $\$ a c c p[1 !=p$ Range $\$$ seqq $]$ ), fitted (model $i), y l$ im=c($5,5)$, xlab="Acceptability",ylab="Predicted Acceptability")

title(expression("Acceptability" ==

bolditalic(f)(PAOT, Iofs)), cex.main=2)

abline $(0,1)$

plot (jitter (pRange $\$ a c c p[1 !=p R a n g e \$ s e q q])$, fitted $($ model $j), y l i m=c(-$ $5,5)$, xlab="Acceptability",ylab="Predicted Acceptability")

title(expression("Acceptability" ==

bolditalic(f)(PAOT, Iofs, paste(Delta, PA0T))), cex.main=2)

abline $(0,1)$

junk <- preplot(model $j$ )

junk <- split(pRange\$accp,pRange\$id)

junk <- lapply (junk, diff)

pRange $\$$ daccp $<-N A$

pRange $\$$ daccp $[1$ != pRange $\$$ seqq $]<-$ unlist (junk)

modelk <- gam(daccp s(paot), data=pRange[nix,])

modell <- $\operatorname{lm}($ daccp $\sim$ dpaot, data=pRange[nix, ])

modelm <- gam(daccp s(paot) + dpaot, data=pRange[nix, ])

anova(modell, modelk, modelm)

modeln <- gam(daccp $\sim \mathrm{s}($ paot $)+\mathrm{s}($ dpaot/paot $)$, data=pRange[nix, ])

\# did 2d spline??

\# consider a factor that is just sign(delta)

anova(modell, modelk, modeln)

nnix $<-$ abs (pRange\$daccp $[$ nix $])<4$

modelnn <- gam(daccp s(paot) +

$s($ dpaot/paot), data=pRange $[$ nix, $][$ nnix, $]$ ) 